Supporting Information for

\title{
Tandem Pd-Catalyzed Intermolecular Allylic Alkylation/Allylic Dearomatization Reaction of Benzoylmethyl Pyridines, Pyrazines and Quinolines
}

\author{
Hui-Jun Zhang ${ }^{\dagger}$, Ze-Peng Yang*, Qing Gu* and Shu-Li You* ${ }^{*}+\star *$ \\ ${ }^{\dagger}$ School of Chemistry and Molecular Engineering, East China University of Science \\ and Technology, 130 Meilong Road, Shanghai 200237, China \\ ${ }^{\ddagger}$ State Key Laboratory of Organometallic Chemistry, Shanghai Institute of Organic \\ Chemistry, Chinese Academy of Sciences, 345 Lingling Lu, Shanghai 200032, China \\ E-mail: $\underline{\text { slyou@sioc.ac.cn }}$
}

Table of Contents

General methods

S2

Optimization of the reaction conditions

S3

$\begin{array}{ll}\text { Experimental details and characterization data } & \text { S5 }\end{array}$

Procedure for the synthesis of intermediate S4 S25

$\begin{array}{ll}\text { X-Ray crystal data of } \mathbf{3 r} \text { and } \mathbf{3 s} & \text { S26 }\end{array}$

$\begin{array}{ll}\text { Copies of NMR spectra } & \text { S30 }\end{array}$

$\begin{array}{ll}\text { References } & \text { S97 }\end{array}$ 


\section{General methods}

Unless stated otherwise, all reactions were carried out in flame-dried glassware under a dry argon atmosphere. All solvents were purified and dried according to standard methods prior to use.

${ }^{\mathbf{1}} \mathbf{H}$ and ${ }^{13} \mathrm{C}$ NMR spectra were recorded on an Agilent instrument (400 MHz and $100 \mathrm{MHz}$, respectively) or an Agilent instrument $(600 \mathrm{MHz}$ and $150 \mathrm{MHz}$, respectively) or a Bruker instrument $(400 \mathrm{MHz}$ and $100 \mathrm{MHz}$, respectively) and internally referenced to tetramethylsilane signal or residual protio solvent signals. ${ }^{19} \mathrm{~F}$ NMR spectra were recorded on an Agilent instrument $(376 \mathrm{MHz})$ or a Bruker instrument $\left(376 \mathrm{MHz}\right.$ ) and referenced relative to $\mathrm{CFCl}_{3}$. Data for ${ }^{1} \mathrm{H} \mathrm{NMR}$ are recorded as follows: chemical shift $(\delta, \mathrm{ppm})$, multiplicity $(\mathrm{s}=$ singlet, $\mathrm{d}=$ doublet, $\mathrm{t}=$ triplet, $\mathrm{m}=$ multiplet or unresolved, $\mathrm{br}=$ broad singlet, coupling constant $(\mathrm{s})$ in $\mathrm{Hz}$, integration). Data for ${ }^{13} \mathrm{C}$ NMR are reported in terms of chemical shift ( $\delta$, ppm).

Substrates $\mathbf{1 a - 1} \mathbf{q}^{[1,2]}, \mathbf{2} \mathbf{a}-\mathbf{2} \mathbf{b}^{[3]}$ were synthesized according to the literature procedure. 


\section{Optimization of the reaction conditions}

Table S1. The effects of triethyl amine equivalents. ${ }^{a}$<smiles>O=C(Cc1ccccn1)c1ccccc1</smiles>

$1 \mathrm{a}$

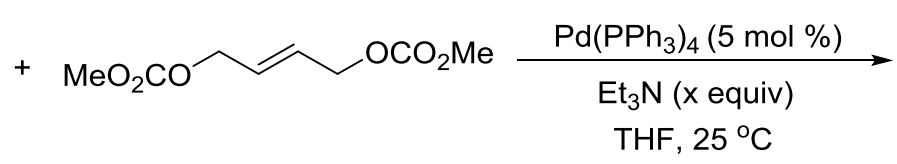

$2 a$

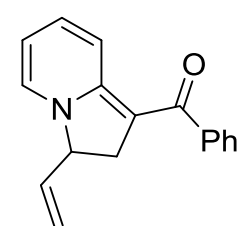

$3 a$

\begin{tabular}{llll}
\hline Entry & $\mathrm{x}($ equiv) & Time $(\mathrm{h})$ & $\mathrm{NMR}$ yield $(\%)^{b}$ \\
\hline 1 & 0.2 & 3 & $68\left(64^{c}\right)$ \\
2 & 0.5 & 2.5 & $72\left(65^{c}\right)$ \\
3 & 1.2 & 1.5 & $79\left(74^{c}\right)$
\end{tabular}

${ }^{a}$ Reaction conditions: $0.4 \mathrm{mmol}$ of 1a, $0.48 \mathrm{mmol}$ of $\mathbf{2 a}, 5 \mathrm{~mol} \%$ of $\mathrm{Pd}\left(\mathrm{PPh}_{3}\right)_{4}$, $\mathrm{x}$ equiv of $\mathrm{Et}_{3} \mathrm{~N}$ in THF ( $4 \mathrm{~mL}$ ) at $25{ }^{\circ} \mathrm{C} .{ }^{b}$ Determined by ${ }^{1} \mathrm{H}$ NMR of the crude reaction mixture using dibromomethane as an internal standard. ${ }^{c}$ Isolated yield.

Table S2. The effects of temperature. ${ }^{a}$<smiles>O=C(Cc1ccccn1)c1ccccc1</smiles>

$1 \mathrm{a}$<smiles>COC(=O)OC/C=C/COC(C)=O</smiles>

2a

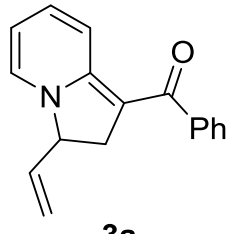

$3 a$

\begin{tabular}{llll}
\hline Entry & $\mathrm{T}\left({ }^{\mathrm{o}} \mathrm{C}\right)$ & Time $(\mathrm{h})$ & NMR yield $(\%)^{b}$ \\
\hline 1 & 0 & 24 & 14 \\
2 & 10 & 24 & 54 \\
3 & 20 & 1 & 68 \\
4 & 25 & 1.5 & 79 \\
5 & 50 & 24 & $<5$
\end{tabular}

${ }^{a}$ Reaction conditions: $0.4 \mathrm{mmol}$ of 1a, $0.48 \mathrm{mmol}$ of $\mathbf{2 a}, 5 \mathrm{~mol} \%$ of $\mathrm{Pd}\left(\mathrm{PPh}_{3}\right)_{4}, 0.48$ mmol of $\mathrm{Et}_{3} \mathrm{~N}$ in THF (4 mL) at T. ${ }^{b}$ Determined by ${ }^{1} \mathrm{H}$ NMR of the crude reaction mixture using dibromomethane as an internal standard. 
Table S3. The effects of $\mathbf{2 a}$ equivalents. ${ }^{a}$

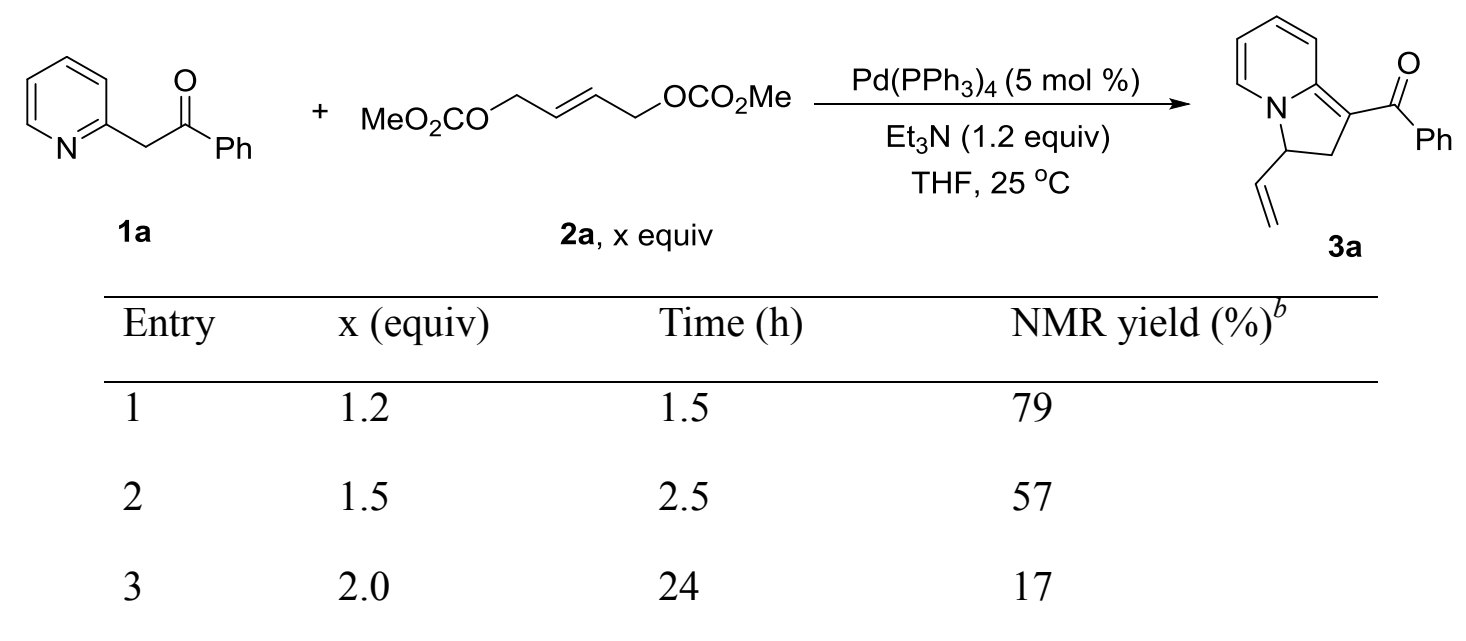

${ }^{a}$ Reaction conditions: $0.4 \mathrm{mmol}$ of $1 \mathrm{a}, \mathrm{x}$ equiv of $\mathbf{2 a}, 5 \mathrm{~mol} \%$ of $\mathrm{Pd}\left(\mathrm{PPh}_{3}\right)_{4}, 0.48$ mmol of $\mathrm{Et}_{3} \mathrm{~N}$ in THF $(4 \mathrm{~mL})$ at $25{ }^{\circ} \mathrm{C}$. ${ }^{b}$ Determined by ${ }^{1} \mathrm{H}$ NMR of the crude reaction mixture using dibromomethane as an internal standard. 


\section{Experimental details and characterization data}

\section{General procedure for the synthesis of substrate 1}

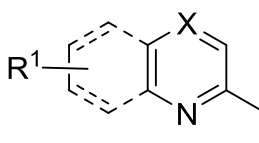

$\mathrm{X}=\mathrm{CH}, \mathrm{N}$

S1

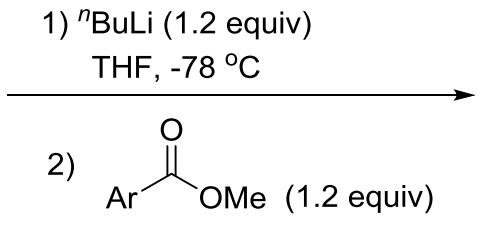

S2

$-78{ }^{\circ} \mathrm{C}-\mathrm{rt}$<smiles>[R][X]1ccc2nc(CC(=O)[Al])ccc2c1</smiles>

1

${ }^{n} \mathrm{BuLi}$ (2.5 $\mathrm{M}$ solution in hexanes, 1.2 equiv) was added dropwise to a stirred solution of $\mathbf{S 1}\left(1.0\right.$ equiv) in THF $(0.2 \mathrm{M})$ at $-78^{\circ} \mathrm{C}$ under Ar. After the resulting mixture was stirred at $-78{ }^{\circ} \mathrm{C}$ for $2 \mathrm{~h}, \mathbf{S 2}$ (1.2 equiv) was added and stirred for another $1 \mathrm{~h}$. Then, the solution was stirred for $12 \mathrm{~h}$ at room temperature. After the reaction was complete (monitored by TLC), the reaction was quenched by brine. The mixture was extracted with EtOAc. The organic layer was washed with brine, dried over $\mathrm{Na}_{2} \mathrm{SO}_{4}$, filtered and concentrated by rotary evaporation. The residue was purified by silica gel column chromatography $(\mathrm{PE} / \mathrm{EtOAc}=10 / 1)$ to afford the desired products 1a, 1d-1o, 1t. Both ketone and enol forms were observed by ${ }^{1} \mathrm{H}$ NMR, and the peaks of enol form were marked with asterisk*. The analytical data of the products are summarized below.<smiles>O=C(Cc1ccccn1)c1ccccc1</smiles>

1a, orange solid, m.p. $=55.3-56.0^{\circ} \mathrm{C}$ (Known compound, see: Muir, C. W.; Kennedy, A. R.; Redmond, J. M.; Watson, A. J. B. Org. Biomol. Chem. 2013, 11, 3337). 1.6 g, $50 \%$ yield $\left(16 \mathrm{mmol}\right.$ scale). ${ }^{1} \mathrm{H}$ NMR $\left(400 \mathrm{MHz}, \mathrm{CDCl}_{3}\right)$ (ketone : enol = 1.4:1) $\delta$ 15.50* (br s, 1H), 8.60-8.52 (m, 1H), 8.29* (d, $J=4.8 \mathrm{~Hz}, 1 \mathrm{H}), 8.12-8.03$ (m, 2H), 7.90-7.80* (m, 2H), 7.68-7.53* (m, 4H), 7.51-7.35 (m, 4H), 7.31 (d, $J=8.0 \mathrm{~Hz}, 1 \mathrm{H})$, 7.21-7.14 (m, 1H), 7.07* (d, $J=8.0 \mathrm{~Hz}, 1 \mathrm{H}), 6.98^{*}(\mathrm{ddd}, J=7.2,5.2,1.2 \mathrm{~Hz}, 1 \mathrm{H})$, $6.08 *(\mathrm{~s}, 1 \mathrm{H}), 4.50(\mathrm{~s}, 2 \mathrm{H})$. 


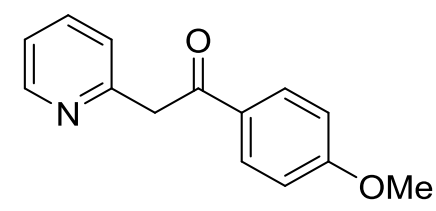

1b, yellow solid, m.p. $=69.7-70.4{ }^{\circ} \mathrm{C}$ (Known compound, see: Muir, C. W.; Kennedy, A. R.; Redmond, J. M.; Watson, A. J. B. Org. Biomol. Chem. 2013, 11, 3337). $1.6 \mathrm{~g}, 44 \%$ yield (16 mmol scale). ${ }^{1} \mathrm{H} \mathrm{NMR}\left(400 \mathrm{MHz}, \mathrm{CDCl}_{3}\right.$ ) (ketone : enol $=5: 1) \delta 15.55^{*}(\mathrm{br} \mathrm{s}, 1 \mathrm{H}), 8.54(\mathrm{~d}, J=4.8 \mathrm{~Hz}, 1 \mathrm{H}), 8.20^{*}(\mathrm{~d}, J=5.2 \mathrm{~Hz}, 1 \mathrm{H}), 8.09$ $7.98(\mathrm{~m}, 2 \mathrm{H}), 7.82-7.77 *(\mathrm{~m}, 2 \mathrm{H}), 7.60(\mathrm{td}, J=7.6,1.6 \mathrm{~Hz}, 1 \mathrm{H}), 7.56-7.51^{*}(\mathrm{~m}, 1 \mathrm{H})$, $7.29(\mathrm{~d}, J=7.6 \mathrm{~Hz}, 1 \mathrm{H}), 7.13(\mathrm{dd}, J=6.8,5.2 \mathrm{~Hz}, 1 \mathrm{H}), 7.00^{*}(\mathrm{~d}, J=8.4 \mathrm{~Hz}, 1 \mathrm{H})$, 6.95-6.87 (m, 2H), 6.95-6.87* (m, 3H), 5.97* (s, 1H), $4.43(\mathrm{~s}, 2 \mathrm{H}), 3.81,3.81 *(\mathrm{~s}, 3 \mathrm{H})$.<smiles>O=C(Cc1cnccn1)c1ccccc1</smiles>

1f, orange solid, m.p. $=87.6-88.6{ }^{\circ} \mathrm{C}$ (Known compound, see: Carver, D. R.; Komin, A. P.; Hubbard, J. S.; Wolfe, J. F. J. Org. Chem. 1981, 46, 294). 1.8 g, $57 \%$ yield (16 mmol scale). ${ }^{1} \mathrm{H}$ NMR (400 MHz, $\left.\mathrm{CDCl}_{3}\right)\left(\right.$ ketone : enol = 2.8:1) $\delta 13.88^{*}(\mathrm{br} \mathrm{s}, 1 \mathrm{H})$, $8.61(\mathrm{~d}, J=1.2 \mathrm{~Hz}, 1 \mathrm{H}), 8.56-8.49(\mathrm{~m}, 1 \mathrm{H}), 8.50-8.42(\mathrm{~m}, 1 \mathrm{H}), 8.50-8.42 *(\mathrm{~m}, 1 \mathrm{H})$, 8.29-8.22* (m, 2H), 8.12-7.98 (m, 2H), 7.88-7.79* (m, 2H), $7.58(\mathrm{t}, J=7.2 \mathrm{~Hz}, 1 \mathrm{H})$, $7.48(\mathrm{t}, J=7.6 \mathrm{~Hz}, 1 \mathrm{H}), 7.48 *(\mathrm{t}, J=7.6 \mathrm{~Hz}, 3 \mathrm{H}), 7.44-7.36(\mathrm{~m}, 1 \mathrm{H}), 6.14 *(\mathrm{~s}, 1 \mathrm{H})$, $4.53(\mathrm{~s}, 2 \mathrm{H})$.<smiles>Cc1ccc(C(=O)Cc2cnccn2)cc1</smiles>

1g, orange solid, m.p. $=73.8-74.2{ }^{\circ} \mathrm{C} .1 .7 \mathrm{~g}, 50 \%$ yield $(16 \mathrm{mmol}$ scale $) .{ }^{1} \mathrm{H}$ NMR $\left(400 \mathrm{MHz}, \mathrm{CDCl}_{3}\right)($ ketone $:$ enol $=5: 1) \delta 13.89 *($ br s, $1 \mathrm{H}), 8.61(\mathrm{~d}, J=1.6 \mathrm{~Hz}, 1 \mathrm{H})$, $8.53(\mathrm{t}, J=2.0 \mathrm{~Hz}, 1 \mathrm{H}), 8.47(\mathrm{~d}, J=2.4 \mathrm{~Hz}, 1 \mathrm{H}), 8.44^{*}(\mathrm{~d}, J=0.8 \mathrm{~Hz}, 1 \mathrm{H}), 8.29-$ $8.22 *(\mathrm{~m}, 2 \mathrm{H}), 7.95(\mathrm{~d}, J=8.0 \mathrm{~Hz}, 2 \mathrm{H}), 7.74 *(\mathrm{~d}, J=8.0 \mathrm{~Hz}, 2 \mathrm{H}), 7.29(\mathrm{~d}, J=8.0 \mathrm{~Hz}$, $2 \mathrm{H}), 7.24 *(\mathrm{~d}, J=8.4 \mathrm{~Hz}, 2 \mathrm{H}), 6.12 *(\mathrm{~s}, 1 \mathrm{H}), 4.51(\mathrm{~s}, 2 \mathrm{H}), 2.42(\mathrm{~s}, 3 \mathrm{H}), 2.40 *(\mathrm{~s}, 3 \mathrm{H})$. ${ }^{13} \mathrm{C} \mathrm{NMR}\left(100 \mathrm{MHz}, \mathrm{CDCl}_{3}\right) \delta 195.5,164.9,154.5,151.5,146.0,144.7,144.3,144.3$, 143.0, 140.4, 139.6, 139.1, 133.9, 132.5, 129.6, 129.3, 128.9, 125.6, 91.6, 45.5, 21.8, 
21.5. IR (thin film): $v_{\max }\left(\mathrm{cm}^{-1}\right)=3332,3053,2950,2915,2378,2325,2300,2120$, 1994, 1918, 1827, 1669, 1599, 1505, 1474, 1449, 1403, 1323, 1285, 1246, 1192, 1121, 1052, 1011, 988, 867, 842, 814, 780, 742, 713, 648, 570, 471. HRMS-ESI calcd for $\mathrm{C}_{13} \mathrm{H}_{13} \mathrm{~N}_{2} \mathrm{O}[\mathrm{M}+\mathrm{H}]^{+}:$213.1022. Found: 213.1021.

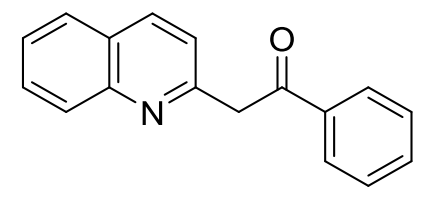

1i, yellow solid, m.p. $=115.4-115.9{ }^{\circ} \mathrm{C}$ (Known compound, see: Wang, T.-L.; Ouyang, G..; He, Y.-M.; Fan, Q.-H. Synlett 2011, 7, 939). 1.3 g, 42\% yield (12.5 mmol scale). ${ }^{1} \mathrm{H}$ NMR (400 MHz, $\mathrm{CDCl}_{3}$ ) (ketone : enol = 1:20) $\delta 15.70 *$ (br s, $1 \mathrm{H}$ ), $8.11(\mathrm{~d}, J=8.0 \mathrm{~Hz}, 3 \mathrm{H}), 8.06(\mathrm{~d}, J=8.0 \mathrm{~Hz}, 2 \mathrm{H}), 7.96^{*}(\mathrm{dd}, J=5.6,1.6 \mathrm{~Hz}, 2 \mathrm{H})$, $7.79(\mathrm{~d}, J=8.4 \mathrm{~Hz}, 2 \mathrm{H}), 7.64 *(\mathrm{~d}, J=8.8 \mathrm{~Hz}, 1 \mathrm{H}), 7.59-7.37 *(\mathrm{~m}, 6 \mathrm{H}), 7.25 *(\mathrm{dd}, J=$ 7.2, 4.8 Hz, 1H), 6.86* (d, $J=9.2 \mathrm{~Hz}, 1 \mathrm{H}), 6.08^{*}(\mathrm{~s}, 1 \mathrm{H}), 4.70(\mathrm{~s}, 2 \mathrm{H})$.<smiles>Cc1ccc2nc(CC(=O)c3ccccc3)ccc2c1</smiles>

11, yellow solid, m.p. $=117.5-118.3{ }^{\circ} \mathrm{C}$ (Known compound, see: Wang, T.-L.; Ouyang, G.; He, Y.-M.; Fan, Q.-H. Synlett 2011, 7, 939). 1.8 g, 49\% yield (14 mmol scale). ${ }^{1} \mathrm{H}$ NMR $\left(400 \mathrm{MHz}, \mathrm{CDCl}_{3}\right.$ ) (ketone : enol = 1:20) $\delta 15.71 *($ br s, $1 \mathrm{H}), 7.98$ 7.90* (m, 2H), 7.59* (d, $J=8.8 \mathrm{~Hz}, 1 \mathrm{H}), 7.45-7.32 *(\mathrm{~m}, 5 \mathrm{H}), 7.29 *(\mathrm{~s}, 1 \mathrm{H}), 6.82 *(\mathrm{~d}$, $J=9.2 \mathrm{~Hz}, 1 \mathrm{H}), 6.02 *(\mathrm{~s}, 1 \mathrm{H}), 4.67(\mathrm{~s}, 2 \mathrm{H}), 2.50(\mathrm{~s}, 3 \mathrm{H}), 2.41 *(\mathrm{~s}, 3 \mathrm{H})$.<smiles>COc1ccc2nc(CC(=O)c3ccccc3)ccc2c1</smiles>

1m, yellow solid, m.p. $=145.8-146.4{ }^{\circ} \mathrm{C}$ (Known compound, see: Wang, T.-L.; Ouyang, G.; He, Y.-M.; Fan, Q.-H. Synlett 2011, 7, 939). 2.0 g, 61\% yield (12 mmol scale). ${ }^{1} \mathrm{H} \mathrm{NMR}\left(400 \mathrm{MHz}, \mathrm{CDCl}_{3}\right.$ ) (ketone : enol = 1:5) $\delta 16.07 *$ (br s, 1H), 8.14$8.08(\mathrm{~m}, 2 \mathrm{H}), 8.00(\mathrm{~d}, J=8.4 \mathrm{~Hz}, 1 \mathrm{H}), 7.98-7.90^{*}(\mathrm{~m}, 2 \mathrm{H}), 7.68^{*}(\mathrm{~d}, J=9.2 \mathrm{~Hz}, 1 \mathrm{H})$, 7.52* $(\mathrm{d}, J=8.8 \mathrm{~Hz}, 1 \mathrm{H}), 7.49-7.39 *(\mathrm{~m}, 3 \mathrm{H}), 7.38-7.35(\mathrm{~m}, 1 \mathrm{H}), 7.22 *(\mathrm{dd}, J=9.2$, 
$2.8 \mathrm{~Hz}, 1 \mathrm{H}), 7.05(\mathrm{~d}, J=2.4 \mathrm{~Hz}, 1 \mathrm{H}), 6.97-6.91 *(\mathrm{~m}, 2 \mathrm{H}), 6.08^{*}(\mathrm{~s}, 1 \mathrm{H}), 4.65(\mathrm{~s}, 2 \mathrm{H})$, $3.91(\mathrm{~s}, 3 \mathrm{H}), 3.87 *(\mathrm{~s}, 3 \mathrm{H})$.

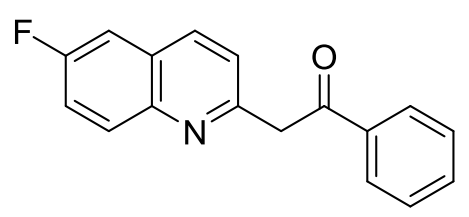

1n, yellow solid, m.p. $=127.9-128.5{ }^{\circ} \mathrm{C}$ (Known compound, see: Wang, T.-L.; Ouyang, G.; He, Y.-M.; Fan, Q.-H. Synlett 2011, 7, 939). 1.2 g, 36\% yield (13 mmol scale). ${ }^{1} \mathrm{H}$ NMR (400 MHz, $\mathrm{CDCl}_{3}$ ) (ketone : enol = 1:6.2) $\delta 15.86^{*}($ br s, $1 \mathrm{H}), 8.13$ $8.08(\mathrm{~m}, 2 \mathrm{H}), 8.08-8.01(\mathrm{~m}, 2 \mathrm{H}), 7.98-7.90 *(\mathrm{~m}, 2 \mathrm{H}), 7.64 *(\mathrm{~d}, J=8.8 \mathrm{~Hz}, 1 \mathrm{H}), 7.59-$ 7.49* (m, 1H), 7.59-7.49 (m, 2H), 7.49-7.38* (m, 3H), 7.49-7.38 (m, 4H), 7.30* (td, $J$ $=8.8,2.8 \mathrm{~Hz}, 1 \mathrm{H}), 7.21 *(\mathrm{dd}, J=8.4,2.8 \mathrm{~Hz}, 1 \mathrm{H}), 6.94 *(\mathrm{~d}, J=9.2 \mathrm{~Hz}, 1 \mathrm{H}), 6.09 *(\mathrm{~s}$, $1 \mathrm{H}), 4.68(\mathrm{~s}, 2 \mathrm{H})$.<smiles>O=C(Cc1ccc2ccc(F)cc2n1)c1ccccc1</smiles>

1p, yellow solid, m.p. $=108.2-109.1^{\circ} \mathrm{C} .1 .4 \mathrm{~g}, 42 \%$ yield $(13 \mathrm{mmol}$ scale $) .{ }^{1} \mathrm{H}$ NMR $\left(400 \mathrm{MHz}, \mathrm{CDCl}_{3}\right)$ (ketone $:$ enol = 1:14.3) $\delta 15.71^{*}($ br s, $1 \mathrm{H}), 8.12-8.03(\mathrm{~m}, 3 \mathrm{H})$, 7.97-7.88* (m, 2H), $7.75(\mathrm{dd}, J=8.8,6.0 \mathrm{~Hz}, 1 \mathrm{H}), 7.66(\mathrm{dd}, J=10.4,2.4 \mathrm{~Hz}, 1 \mathrm{H})$, $7.57 *(\mathrm{~d}, J=9.2 \mathrm{~Hz}, 1 \mathrm{H}), 7.50-7.39 *(\mathrm{~m}, 4 \mathrm{H}), 7.37$ (d, $J=8.4 \mathrm{~Hz}, 1 \mathrm{H}), 7.29$ (dd, $J=$ 8.4, $2.4 \mathrm{~Hz}, 1 \mathrm{H}), 7.13^{*}(\mathrm{dd}, J=9.6,2.4 \mathrm{~Hz}, 1 \mathrm{H}), 6.96^{*}(\mathrm{td}, J=8.4,2.4 \mathrm{~Hz}, 1 \mathrm{H})$, $6.78^{*}(\mathrm{~d}, J=9.2 \mathrm{~Hz}, 1 \mathrm{H}), 6.06 *(\mathrm{~s}, 1 \mathrm{H}), 4.66(\mathrm{~s}, 2 \mathrm{H}) .{ }^{19} \mathrm{~F} \mathrm{NMR}\left(376 \mathrm{MHz}, \mathrm{CDCl}_{3}\right) \delta-$ 110.9(m), -113.2(m). ${ }^{13} \mathrm{C}$ NMR (100 MHz, $\left.\mathrm{CDCl}_{3}\right) \delta 183.0,164.0(\mathrm{~d}, J=249.5 \mathrm{~Hz})$, 157.0, 154.8, 139.8 (d, $J=123.0 \mathrm{~Hz}), 139.3,136.5(\mathrm{~d}, J=185.0 \mathrm{~Hz}), 135.7,133.5$, $130.6,129.6(\mathrm{~d}, J=101.0 \mathrm{~Hz}), 128.8(\mathrm{~d}, J=79.0 \mathrm{~Hz}), 128.4,126.7,121.6(\mathrm{~d}, J=27.0$ $\mathrm{Hz}), 121.5(\mathrm{~d}, J=27.0 \mathrm{~Hz}), 120.3(\mathrm{~d}, J=17.0 \mathrm{~Hz}) 117.0,116.7,112.8(\mathrm{~d}, J=20.3$ $\mathrm{Hz}), 112.7(\mathrm{~d}, J=23.7 \mathrm{~Hz}), 104.6(\mathrm{~d}, J=24.4 \mathrm{~Hz}) 90.7,49.3$. IR (thin film): $v_{\max }\left(\mathrm{cm}^{-1}\right)$ $=3076,3053,2954,2920,2853,2387,2304,2116,1994,1877,1817,1626,1584$, 1534, 1510, 1447, 1404, 1322, 1300, 1274, 1207, 1177, 1147, 1105, 1062, 1023, 985, 942, 868, 835, 756, 726, 686, 656. HRMS-ESI calcd for $\mathrm{C}_{17} \mathrm{H}_{13} \mathrm{FNO}[\mathrm{M}+\mathrm{H}]^{+}$: 266.0976. Found: 266.0973. 


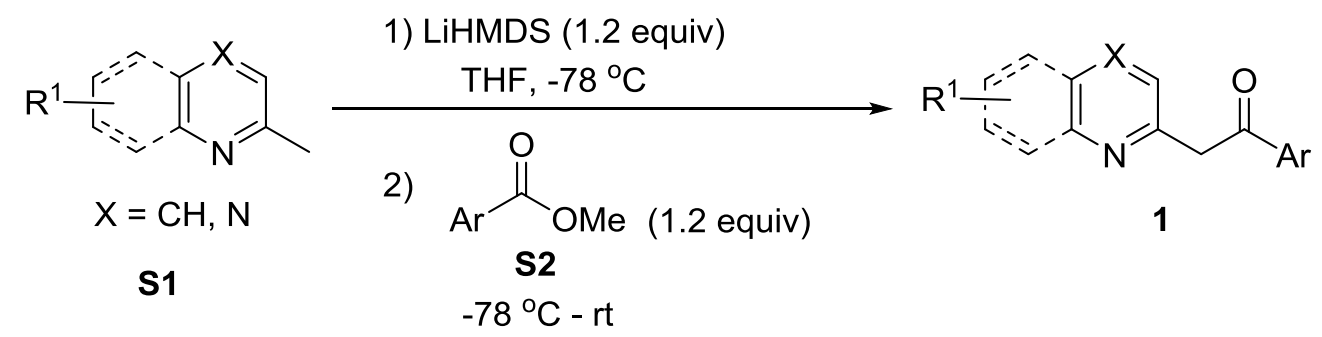

LiHMDS (1.0 M solution in THF, 1.2 equiv) was added dropwise to a stirred solution of $\mathbf{S 1}\left(1.0\right.$ equiv) in THF $(0.2 \mathrm{M})$ at $-78^{\circ} \mathrm{C}$ under Ar. After the resulting mixture was stirred at $-78{ }^{\circ} \mathrm{C}$ for $2 \mathrm{~h}, \mathbf{S 2}$ (1.2 equiv) was added and stirred for another $1 \mathrm{~h}$. Then, the solution was stirred for $12 \mathrm{~h}$ at room temperature. After the reaction was complete (monitored by TLC), the reaction was quenched by brine. The mixture was extracted with EtOAc. The organic layer was washed with brine, dried over $\mathrm{Na}_{2} \mathrm{SO}_{4}$, filtered and concentrated by rotary evaporation. The residue was purified by silica gel column chromatography $(\mathrm{PE} / \mathrm{EtOAc}=10 / 1)$ to afford the desired products $\mathbf{1 c}-\mathbf{1 e}, \mathbf{1 h}, \mathbf{1 j}-\mathbf{1 k}$, 1o, 1q. Both ketone and enol forms were observed by ${ }^{1} \mathrm{H}$ NMR, and the peaks of enol form were marked with asterisk*. The analytical data of the products are summarized below.<smiles>O=C(Cc1ccccn1)c1ccc(Cl)cc1</smiles>

1c, yellow solid, m.p. $=88.2-89.1{ }^{\circ} \mathrm{C}$ (Known compound, see: Muir, C. W.; Kennedy, A. R.; Redmond, J. M.; Watson, A. J. B. Org. Biomol. Chem. 2013, 11, 3337). 1.1 g, $37 \%$ yield (13 mmol scale). ${ }^{1} \mathrm{H}$ NMR (400 $\left.\mathrm{MHz}, \mathrm{CDCl}_{3}\right)$ (ketone : enol = 1:1.2) $\delta$ 15.57* (br s, 1H), 8.58-8.51 (m, 1H), 8.27* (d, $J=5.2 \mathrm{~Hz}, 1 \mathrm{H}), 8.05-7.97$ (m, 2H), 7.81-7.72* (m, 2H), 7.68-7.56 (m, 1H), 7.68-7.56* (m, 1H), 7.46-7.39 (m, 2H), 7.39$7.32 *(\mathrm{~m}, 2 \mathrm{H}), 7.29(\mathrm{~d}, J=8.0 \mathrm{~Hz}, 1 \mathrm{H}), 7.17(\mathrm{dd}, J=6.8,5.2 \mathrm{~Hz}, 1 \mathrm{H}), 7.05^{*}(\mathrm{~d}, J=$ 8.0 Hz, 1H), 7.01-6.93* (m, 1H), 6.03* (s, 1H), 4.45 (s, 2H).<smiles>O=C(Cc1ccccn1)c1ccc(Br)cc1</smiles> 
1d, orange soild, m.p. $=81.5-82.1{ }^{\circ} \mathrm{C}$ (Known compound, see: Muir, C. W.; Kennedy, A. R.; Redmond, J. M.; Watson, A. J. B. Org. Biomol. Chem. 2013, 11, 3337). 445 mg, $54 \%$ yield (3 mmol scale). ${ }^{1} \mathrm{H} \mathrm{NMR}\left(400 \mathrm{MHz}, \mathrm{CDCl}_{3}\right.$ ) (ketone : enol =1:1.3) $\delta$ $15.57^{*}$ (br s, 1H), 8.55 (d, $\left.J=4.0 \mathrm{~Hz}, 1 \mathrm{H}\right), 8.28^{*}(\mathrm{~d}, J=5.2 \mathrm{~Hz}, 1 \mathrm{H}), 7.98-7.89$ (m, $2 \mathrm{H}), 7.71^{*}(\mathrm{~d}, J=8.8 \mathrm{~Hz}, 2 \mathrm{H}), 7.67-7.57(\mathrm{~m}, 3 \mathrm{H}), 7.67-7.57 *(\mathrm{~m}, 1 \mathrm{H}), 7.53 *(\mathrm{~d}, J=$ $8.4 \mathrm{~Hz}, 2 \mathrm{H}), 7.29$ (d, $J=8.0 \mathrm{~Hz}, 1 \mathrm{H}), 7.18(\mathrm{dd}, J=6.8,5.2 \mathrm{~Hz}, 1 \mathrm{H}), 7.06^{*}(\mathrm{~d}, J=8.0$ $\mathrm{Hz}, 1 \mathrm{H}), 6.99 *(\mathrm{dd}, J=6.4,6.0 \mathrm{~Hz}, 1 \mathrm{H}), 6.05 *(\mathrm{~s}, 1 \mathrm{H}), 4.45$ (s, 2H).

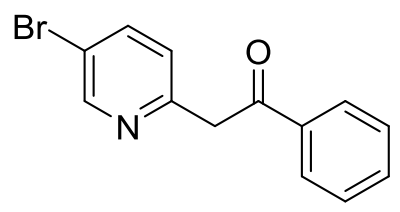

1e, yellow solid, m.p. $=91.2-92.0{ }^{\circ} \mathrm{C} .2 .1 \mathrm{~g}, 33 \%$ yield $(23 \mathrm{mmol}$ scale $) .{ }^{1} \mathrm{H}$ NMR $\left(400 \mathrm{MHz}, \mathrm{CDCl}_{3}\right)($ ketone $:$ enol $=2: 1) \delta 14.35^{*}($ br s, $1 \mathrm{H}), 8.62(\mathrm{~d}, J=2.4 \mathrm{~Hz}, 1 \mathrm{H})$, 8.43* (d, $J=2.4 \mathrm{~Hz}, 1 \mathrm{H}), 8.09-7.99$ (m, 2H), 7.86-7.80* (m, 2H), 7.77 (dd, $J=8.4$, $2.4 \mathrm{~Hz}, 1 \mathrm{H}), 7.72^{*}(\mathrm{dd}, J=8.8,2.4 \mathrm{~Hz}, 1 \mathrm{H}), 7.57(\mathrm{t}, J=7.2 \mathrm{~Hz}, 1 \mathrm{H}), 7.47$ (t, $J=7.6$ $\mathrm{Hz}, 2 \mathrm{H}), 7.44-7.36^{*}(\mathrm{~m}, 3 \mathrm{H}), 7.22(\mathrm{~d}, J=8.0 \mathrm{~Hz}, 1 \mathrm{H}), 6.99 *(\mathrm{~d}, J=8.4 \mathrm{~Hz}, 1 \mathrm{H})$, 6.06* (s, 1H), $4.46(\mathrm{~s}, 2 \mathrm{H}) .{ }^{13} \mathrm{C} \mathrm{NMR}\left(100 \mathrm{MHz}, \mathrm{CDCl}_{3}\right) \delta 196.5,162.8,157.3,153.9$, $150.7,146.7,139.8,139.3,136.4$, 135.8, 133.6, 129.7, 128.8, 128.5, 125.7, 125.6, 123.0, 119.3, 114.4, 94.5, 47.7. IR (thin film): $v_{\max }\left(\mathrm{cm}^{-1}\right)=3053,2669,2348,2124$, 2089, 1990, 1895, 1846, 1723, 1617, 1577, 1533, 1491, 1464, 1448, 1428, 1372, 1349, 1268, 1200, 1182, 1147, 1128, 1078, 1057, 1028, 1005, 924, 856, 837, 762, 730, 685, 644. HRMS-ESI calcd for $\mathrm{C}_{13} \mathrm{H}_{11} \mathrm{BrNO}[\mathrm{M}+\mathrm{H}]^{+}:$276.0019. Found: 276.0015.

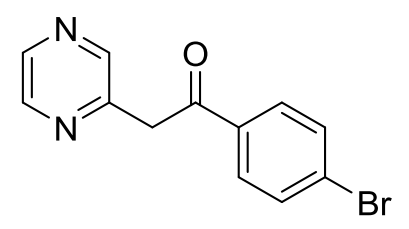

1h, orange solid, m.p. $=107.0-108.1{ }^{\circ} \mathrm{C} .1 .8 \mathrm{~g}, 47 \%$ yield $(14 \mathrm{mmol}$ scale $) .{ }^{1} \mathrm{H}$ NMR $\left(400 \mathrm{MHz}, \mathrm{CDCl}_{3}\right)\left(\right.$ ketone $:$ enol = 1:9.1) $\delta 13.87^{*}(\mathrm{br} \mathrm{s}, 1 \mathrm{H}), 8.61(\mathrm{~s}, 1 \mathrm{H}), 8.54-8.51$ (m, 1H), $8.49(\mathrm{~d}, J=2.4 \mathrm{~Hz}, 1 \mathrm{H}), 8.45^{*}(\mathrm{~s}, 1 \mathrm{H}), 8.34-8.23^{*}(\mathrm{~m}, 2 \mathrm{H}), 7.91(\mathrm{~d}, J=8.4$ $\mathrm{Hz}, 2 \mathrm{H}), 7.69 *(\mathrm{~d}, J=8.4 \mathrm{~Hz}, 2 \mathrm{H}), 7.62(\mathrm{~d}, J=8.4 \mathrm{~Hz}, 2 \mathrm{H}), 7.54 *(\mathrm{~d}, J=8.4 \mathrm{~Hz}, 2 \mathrm{H})$, 6.11* (s, 1H), 4.48 (s, 2H). ${ }^{13} \mathrm{C} \mathrm{NMR}\left(100 \mathrm{MHz}, \mathrm{CDCl}_{3}\right) \delta$ 194.9, 163.5, 154.1, 151.0, 145.9, 144.4, 144.3, 143.2, 139.8, 139.6, 135.0, 134.2, 132.3, 131.84, 131.79, 
131.6, 130.3, 129.1, 127.2, 124.4, 92.5, 45.5. IR (thin film): $v_{\max }\left(\mathrm{cm}^{-1}\right)=3076,3042$, 2678, 2645, 2378, 2327, 2298, 2247, 2200, 2115, 1998, 1916, 1859, 1806, 1767, 1680, 1629, 1584, 1504, 1482, 1445, 1406, 1370, 1316, 1280, 1232, 1182, 1163, 1138, 1073, 1046, 1009, 930, 881, 826, 782, 743, 716, 654, 627. HRMS-ESI calcd for $\mathrm{C}_{12} \mathrm{H}_{10} \mathrm{BrN}_{2} \mathrm{O}[\mathrm{M}+\mathrm{H}]^{+}:$276.9971. Found: 276.9973.

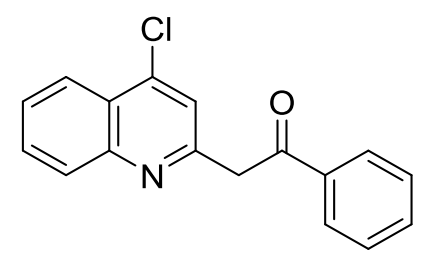

1j, orange solid, m.p. $=78.9-80.1^{\circ} \mathrm{C} .2 .2 \mathrm{~g}, 34 \%$ yield $(23 \mathrm{mmol}$ scale $) .{ }^{1} \mathrm{H}$ NMR $\left(400 \mathrm{MHz}, \mathrm{CDCl}_{3}\right)($ ketone $:$ enol $=1: 11.1) \delta 15.60 *($ br s, $1 \mathrm{H}), 8.18(\mathrm{~d}, J=8.0 \mathrm{~Hz}$, $1 \mathrm{H}), 8.08(\mathrm{dd}, J=8.8,7.6 \mathrm{~Hz}, 3 \mathrm{H}), 7.99-7.85^{*}(\mathrm{~m}, 3 \mathrm{H}), 7.77-7.70(\mathrm{~m}, 1 \mathrm{H}), 7.57 *(\mathrm{td}$, $J=8.0,1.2 \mathrm{~Hz}, 1 \mathrm{H}), 7.54-7.39 *(\mathrm{~m}, 4 \mathrm{H}), 7.31 *(\mathrm{td}, J=7.6,1.2 \mathrm{~Hz}, 1 \mathrm{H}), 6.97 *(\mathrm{~d}, J=$ $2.0 \mathrm{~Hz}, 1 \mathrm{H}), 6.01^{*}(\mathrm{~s}, 1 \mathrm{H}), 4.65(\mathrm{~s}, 2 \mathrm{H}) .{ }^{13} \mathrm{C} \mathrm{NMR}\left(100 \mathrm{MHz}, \mathrm{CDCl}_{3}\right) \delta 182.7,154.0$, $141.7,139.5,139.1,133.7,132.0,130.7,130.6,129.4,128.8,128.5,127.4,126.7$, $124.7,124.4,124.1,122.4,122.0,121.6,119.3,90.3,48.9$. IR (thin film): $v_{\max }\left(\mathrm{cm}^{-1}\right)=$ 3086, 3061, 3024, 2295, 2116, 2078, 1998, 1928, 1893, 1807, 1714, 1622, 1584, 1542, 1448, 1416, 1374, 1350, 1323, 1277, 1211, 1183, 1155, 1101, 1063, 1028, 1002, 985, 947, 851, 799, 755, 726, 682, 655, 635. HRMS-ESI calcd for $\mathrm{C}_{17} \mathrm{H}_{13} \mathrm{ClNO}[\mathrm{M}+\mathrm{H}]^{+}$: 282.0680. Found: 282.0676 .<smiles>O=C(Cc1cc(Br)c2ccccc2n1)c1ccccc1</smiles>

1k, orange solid, m.p. $=87.1-87.6{ }^{\circ} \mathrm{C} .861 \mathrm{mg}, 53 \%$ yield $(5 \mathrm{mmol} \mathrm{scale}) .{ }^{1} \mathrm{H}$ NMR $\left(400 \mathrm{MHz}, \mathrm{CDCl}_{3}\right)($ ketone $:$ enol $=1: 10) \delta 15.59 *($ br s, $1 \mathrm{H}), 8.16(\mathrm{~d}, J=8.0 \mathrm{~Hz}, 1 \mathrm{H})$, $8.11(\mathrm{~d}, J=7.2 \mathrm{~Hz}, 2 \mathrm{H}), 8.06(\mathrm{~d}, J=8.4 \mathrm{~Hz}, 1 \mathrm{H}), 7.98-7.85^{*}(\mathrm{~m}, 3 \mathrm{H}), 7.78-7.73(\mathrm{~m}$, $2 \mathrm{H}), 7.63-7.54 *(\mathrm{~m}, 1 \mathrm{H}), 7.52-7.40 *(\mathrm{~m}, 4 \mathrm{H}), 7.37-7.28 *(\mathrm{~m}, 1 \mathrm{H}), 7.24 *(\mathrm{~d}, J=6.0$ $\mathrm{Hz}, 1 \mathrm{H}), 6.02 *(\mathrm{~d}, J=3.6 \mathrm{~Hz}, 1 \mathrm{H}), 4.67(\mathrm{~s}, 2 \mathrm{H}) .{ }^{13} \mathrm{C} \mathrm{NMR}\left(100 \mathrm{MHz}, \mathrm{CDCl}_{3}\right) \delta 182.8$, 154.0, 139.2, 139.1, 133.1, 132.1, 130.8, 128.9, 128.5, 127.5, 126.7, 125.6, 124.6, 123.1, 119.4, 90.1. IR (thin film): $v_{\max }\left(\mathrm{cm}^{-1}\right)=3740,3670,3058,3024,2672,2355$, 
2321, 2119, 1998, 1891, 1837, 1801, 1767, 1619, 1583, 1538, 1450, 1416, 1363, 1317, 1279, 1208, 1155, 1091, 1065, 1029, 979, 930, 854, 806, 750, 723, 680, 655, 627, 579, 518, 486, 442. HRMS-ESI calcd for $\mathrm{C}_{17} \mathrm{H}_{13} \mathrm{BrNO}[\mathrm{M}+\mathrm{H}]^{+}$: 326.0175. Found: 326.0170 .

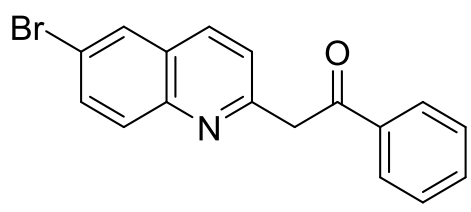

1o, yellow solid, m.p. $=141.9-142.8{ }^{\circ} \mathrm{C} .1 .9 \mathrm{~g}, 59 \%$ yield $(10 \mathrm{mmol}$ scale $) .{ }^{1} \mathrm{H}$ NMR $\left(400 \mathrm{MHz}, \mathrm{CDCl}_{3}\right)\left(\right.$ ketone : enol = 1:1.4) $\delta 15.65^{*}$ (br s, 1H), $8.08(\mathrm{~d}, J=7.2 \mathrm{~Hz}$, 2H), $7.98(\mathrm{~d}, J=8.8 \mathrm{~Hz}, 1 \mathrm{H}), 7.96-7.89 *(\mathrm{~m}, 2 \mathrm{H}), 7.73(\mathrm{dd}, J=8.8,2.0 \mathrm{~Hz}, 1 \mathrm{H})$, $7.60^{*}(\mathrm{~d}, J=2.0 \mathrm{~Hz}, 1 \mathrm{H}), 7.56^{*}(\mathrm{dd}, J=8.8,2.0 \mathrm{~Hz}, 1 \mathrm{H}), 7.50^{*}(\mathrm{~d}, J=9.2 \mathrm{~Hz}, 1 \mathrm{H})$, 7.46-7.39* (m, 3H), 7.32* (d, $J=8.8 \mathrm{~Hz}, 1 \mathrm{H}), 6.84^{*}(\mathrm{~d}, J=9.2 \mathrm{~Hz}, 1 \mathrm{H}), 6.05^{*}$ (s, 1H), 4.65 (s, 2H). ${ }^{13} \mathrm{C}$ NMR (100 MHz, $\left.\mathrm{CDCl}_{3}\right) \delta 182.7,156.3,154.4,146.6,139.1$, $137.4,135.5,134.9,133.9,133.6,133.1,130.8,130.7,129.8,129.7,128.8,128.8$, $128.5,126.7,124.9,123.5,123.1,120.5,116.6,90.9,49.3 . \mathrm{IR}($ thin film $): v_{\max }\left(\mathrm{cm}^{-1}\right)=$ 3081, 3054, 3032, 2959, 2921, 2851, 2626, 2376, 2312, 2175, 2120, 1993, 1891, 1811, 1784, 1763, 1716, 1632, 1579, 1550, 1527, 1440, 1419, 1378, 1335, 1306, 1262, 1222 , 1187, 1156, 1085, 1065, 1020, 950, 926, 882, 852, 814, 789, 759, 735, 709, 690, 658, 629. HRMS-ESI calcd for $\mathrm{C}_{17} \mathrm{H}_{13} \mathrm{BrNO}[\mathrm{M}+\mathrm{H}]^{+}$: 326.0175. Found: 326.0170 .

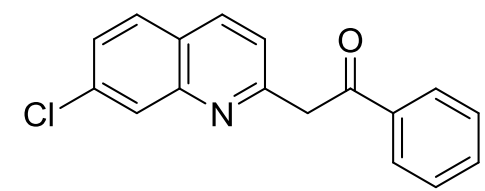

1q, yellow solid, m.p. $=146.1-146.7{ }^{\circ} \mathrm{C} .2 .0 \mathrm{~g}, 58 \%$ yield $(12 \mathrm{mmol}$ scale $) .{ }^{1} \mathrm{H}$ NMR $\left(400 \mathrm{MHz}, \mathrm{CDCl}_{3}\right)$ (ketone $:$ enol $\left.=1: 11.1\right) \delta 15.63^{*}($ br s, $1 \mathrm{H}), 8.13-8.08(\mathrm{~m}, 2 \mathrm{H})$, 8.08-8.03 (m, 1H), 7.97-7.89* (m, 2H), $7.72(\mathrm{~d}, J=8.8 \mathrm{~Hz}, 1 \mathrm{H}), 7.59 *(\mathrm{~d}, J=9.2 \mathrm{~Hz}$, $1 \mathrm{H}), 7.50-7.39 *(\mathrm{~m}, 5 \mathrm{H}), 7.19 *(\mathrm{dd}, J=8.4,2.0 \mathrm{~Hz}, 1 \mathrm{H}), 6.84 *(\mathrm{~d}, J=9.2 \mathrm{~Hz}, 1 \mathrm{H})$, 6.08* (s, 1H), 4.67 (s, 1H). ${ }^{13} \mathrm{C}$ NMR (100 MHz, $\left.\mathrm{CDCl}_{3}\right) \delta 183.0,154.7,139.3,139.2$, $136.8,136.4$, 135.5, 133.6, 130.7, 128.9, 128.8, 128.7, 128.5, 128.2, 127.5, 126.8, $124.5,122.6,122.5,122.0,118.5,90.9,49.4$. IR (thin film): $v_{\max }\left(\mathrm{cm}^{-1}\right)=3087,3036$, 2382, 2321, 2303, 2174, 2114, 1994, 1939, 1886, 1799, 1622, 1590, 1539, 1493, 1449, 1424, 1395, 1335, 1320, 1295, 1193, 1175, 1148, 1130, 1096, 1072, 1025, 974, 934, 
914, 856, 833, 788, 755, 719, 679, 650. HRMS-ESI calcd for $\mathrm{C}_{17} \mathrm{H}_{13} \mathrm{CINO}[\mathrm{M}+\mathrm{H}]^{+}$: 282.0680. Found: 282.0677. 


\section{General procedure for the synthesis of substrates 2}

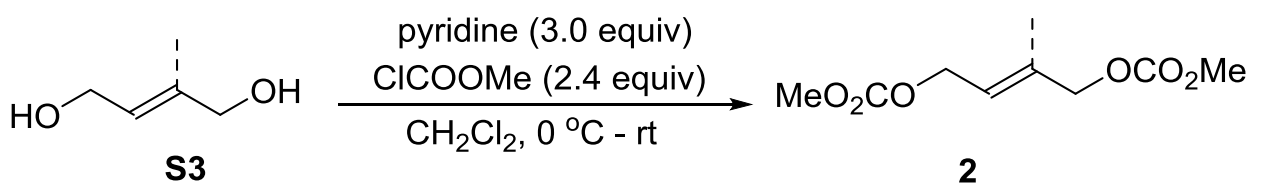

To a solution of $\mathbf{S 3}$ (1.0 equiv) and pyridine (3.0 equiv) in $\mathrm{CH}_{2} \mathrm{Cl}_{2}(0.4 \mathrm{M})$ was added dropwisely methyl chloroformate $\left(2.4\right.$ equiv) at $0{ }^{\circ} \mathrm{C}$. After the addition was completed, the reaction mixture was slowly warmed to room temperature and stirred overnight. After the reaction was complete (monitored by TLC), the reaction was quenched with $2 \mathrm{M} \mathrm{HCl}$. The mixture was extracted with $\mathrm{CH}_{2} \mathrm{Cl}_{2}$. The organic layer was washed with brine, dried over $\mathrm{Na}_{2} \mathrm{SO}_{4}$, filtered and concentrated by rotary evaporation. The residue was purified by silica gel column chromatography $(\mathrm{PE} / \mathrm{EtOAc}=5 / 1)$ to afford the desired product 2.

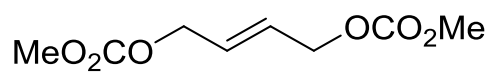

2a, white solid, m.p. $=62.7-63.2{ }^{\circ} \mathrm{C}$ (Known compound, see: He, H.; Liu, W.-B.; Dai, L.-X.; You, S.-L. Angew. Chem. Int. Ed. 2010, 49, 1496). 2.3 g, 71\% yield (16 mmol scale). ${ }^{1} \mathrm{H}$ NMR $\left(400 \mathrm{MHz}, \mathrm{CDCl}_{3}\right) \delta 5.91$ (ddd, $\left.J=4.4,3.2,1.6 \mathrm{~Hz}, 2 \mathrm{H}\right), 4.68-4.63$ $(\mathrm{m}, 4 \mathrm{H}), 3.80(\mathrm{~s}, 6 \mathrm{H})$.

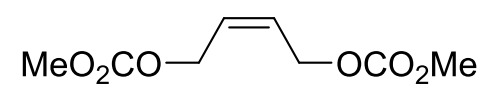

2b, colorless oil (Known compound, see: Wang, L.; Li, P.-F.; Menche, D. Angew. Chem. Int. Ed. 2010, 49, 9270). 2.5 g, $78 \%$ yield (16 mmol scale). ${ }^{1} \mathrm{H}$ NMR (400 $\left.\mathrm{MHz}, \mathrm{CDCl}_{3}\right) \delta 5.81(\mathrm{t}, J=4.0 \mathrm{~Hz}, 2 \mathrm{H}), 4.76(\mathrm{~d}, J=5.2 \mathrm{~Hz}, 4 \mathrm{H}), 3.79(\mathrm{~s}, 6 \mathrm{H})$. 
$\mathrm{MeO}_{2} \mathrm{CO} \sim \mathrm{OCO}_{2} \mathrm{Me}$

2c, white solid, m.p. $=37.7-37.9{ }^{\circ} \mathrm{C} .3 .2 \mathrm{~g}, 93 \%$ yield $\left(16 \mathrm{mmol}\right.$ scale) ${ }^{1} \mathrm{H}$ NMR (400 $\left.\mathrm{MHz}, \mathrm{CDCl}_{3}\right) \delta 5.77-5.61(\mathrm{~m}, 1 \mathrm{H}), 4.70(\mathrm{~d}, J=6.4 \mathrm{~Hz}, 2 \mathrm{H}), 4.56(\mathrm{~s}, 2 \mathrm{H}), 3.80(\mathrm{~s}, 3 \mathrm{H})$, 3.79 (s, 3H), 1.77 (s, 3H). ${ }^{13} \mathrm{C}$ NMR (100 MHz, $\left.\mathrm{CDCl}_{3}\right) \delta 155.8,155.6,136.0,121.7$, 71.9, 63.9, 55.0, 54.9, 14.1. IR (thin film): $v_{\max }\left(\mathrm{cm}^{-1}\right)=3019,2961,2865,1742,1588$, 1441, 1401, 1317, 1251, 1004, 932, 856, 784, 601, 567, 501, 443. HRMS-ESI calcd for $\mathrm{C}_{9} \mathrm{H}_{14} \mathrm{NaO}_{6}[\mathrm{M}+\mathrm{Na}]^{+}$: 241.0683. Found: 241.0683 .

\section{General procedure for Pd-catalyzed tandem allylic substitution reactions}

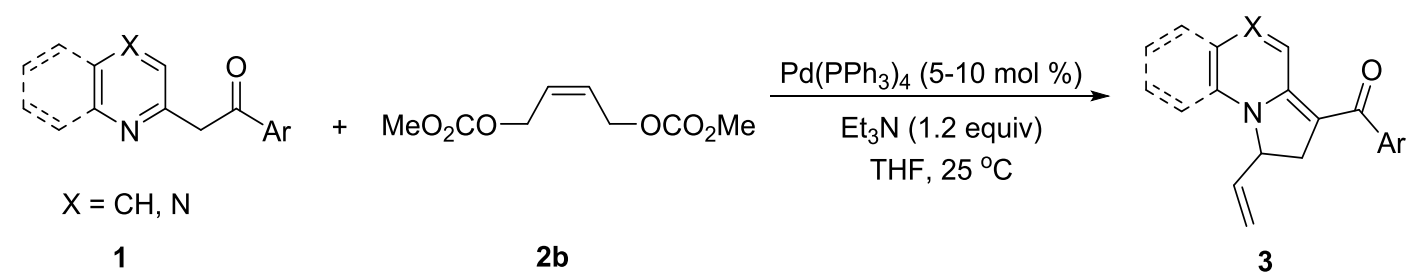

A flame-dried Schlenk tube was cooled to room temperature and filled with argon. To this flask were added $\mathbf{1}$ (0.4 mmol, 1.0 equiv), allylic carbonate $\mathbf{2 b}(0.48 \mathrm{mmol}, 1.2$ equiv), $\mathrm{Pd}\left(\mathrm{PPh}_{3}\right)_{4}(0.02 \mathrm{mmol}, 5 \mathrm{~mol} \%), \mathrm{Et}_{3} \mathrm{~N}(0.48 \mathrm{mmol}, 1.2$ equiv) and THF (4 $\mathrm{mL}$ ). The reaction mixture was stirred at room temperature. After the reaction was complete (monitored by TLC), brine $(20 \mathrm{~mL})$ was added to quench the reaction. This reaction mixture was extracted with EtOAc $(3 \times 15 \mathrm{~mL})$. The extracts were combined, dried over $\mathrm{Na}_{2} \mathrm{SO}_{4}$, and evaporated under reduced pressure. The isomer ratio (3f-3f', 3g-3g' and $\mathbf{3 h}-\mathbf{3} \mathbf{h}^{\prime}$ ) was determined by ${ }^{1} \mathrm{H}$ NMR of the crude reaction mixture. The residue was purified by silica gel column chromatography $\left(\mathrm{CH}_{2} \mathrm{Cl}_{2} / \mathrm{MeOH}=20 / 1\right)$ to afford the desired product 3 . The analytical data of the products are summarized below. 


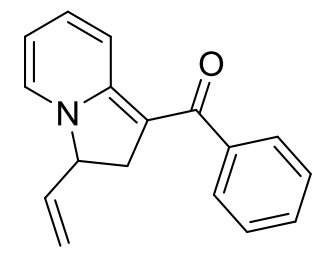

3a, red solid, m.p. $=78.4-79.3{ }^{\circ} \mathrm{C}$ (Known compound, see: Yang, Z.-P.; Wu, Q.-F.; You, S.-L. Angew. Chem. Int. Ed. 2014, 53, 6986). 73 mg, 73\% yield. ${ }^{1}$ H NMR (400 $\left.\mathrm{MHz}, \mathrm{CDCl}_{3}\right) \delta 8.30(\mathrm{~s}, 1 \mathrm{H}), 7.65-7.03(\mathrm{~m}, 7 \mathrm{H}), 6.20(\mathrm{~s}, 1 \mathrm{H}), 5.98-5.82(\mathrm{~m}, 1 \mathrm{H})$, $5.35(\mathrm{~d}, J=9.6 \mathrm{~Hz}, 1 \mathrm{H}), 5.33(\mathrm{~d}, J=17.2 \mathrm{~Hz}, 1 \mathrm{H}), 4.77(\mathrm{~m}, 1 \mathrm{H}), 3.42-3.26(\mathrm{~m}, 1 \mathrm{H})$, $2.92(\mathrm{dd}, J=14.4,8.4 \mathrm{~Hz}, 1 \mathrm{H})$.

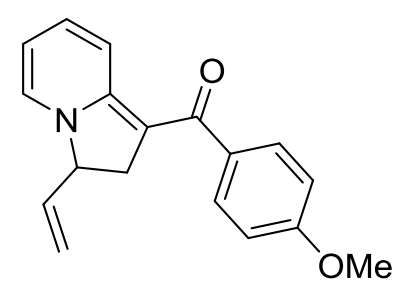

3b, red solid, m.p. $=108.6-110.3{ }^{\circ} \mathrm{C} .60 .3 \mathrm{mg}, 54 \%$ yield. ${ }^{1} \mathrm{H}$ NMR $\left(400 \mathrm{MHz}, \mathrm{CDCl}_{3}\right)$ $\delta 7.54(\mathrm{~d}, J=7.6 \mathrm{~Hz}, 3 \mathrm{H}), 7.47-6.91(\mathrm{~m}, 2 \mathrm{H}), 6.88(\mathrm{~d}, J=8.8 \mathrm{~Hz}, 2 \mathrm{H}), 6.14(\mathrm{~s}, 1 \mathrm{H})$, $5.92(\mathrm{ddd}, J=17.2,10.0,8.8 \mathrm{~Hz}, 1 \mathrm{H}), 5.36(\mathrm{~d}, J=9.6 \mathrm{~Hz}, 1 \mathrm{H}), 5.34(\mathrm{~d}, J=16.8 \mathrm{~Hz}$, $1 \mathrm{H}), 4.76(\mathrm{~m}, 1 \mathrm{H}), 3.82(\mathrm{~s}, 3 \mathrm{H}), 3.36(\mathrm{dd}, J=14.4,10.8 \mathrm{~Hz}, 1 \mathrm{H}), 2.96(\mathrm{dd}, J=14.4$, $8.4 \mathrm{~Hz}, 1 \mathrm{H}) .{ }^{13} \mathrm{C} \mathrm{NMR}\left(100 \mathrm{MHz}, \mathrm{CDCl}_{3}\right) \delta 184.0,160.0,154.8,137.6,136.1,135.9$, $134.2,128.8,120.2,119.4,113.2,109.7,96.9,68.0,55.2,35.8$. IR (thin film): $v_{\max }\left(\mathrm{cm}^{-1}\right)=3063,3008,2945,2920,2844,1622,1602,1578,1523,1472,1442,1420$, 1404, 1331, 1294, 1243, 1182, 1163, 1128, 1106, 1090, 1018, 935, 868, 838, 802, 745, 690, 594, 527, 460. HRMS-ESI calcd for $\mathrm{C}_{18} \mathrm{H}_{18} \mathrm{NO}_{2}[\mathrm{M}+\mathrm{H}]^{+}:$280.1332. Found: 280.1327 .

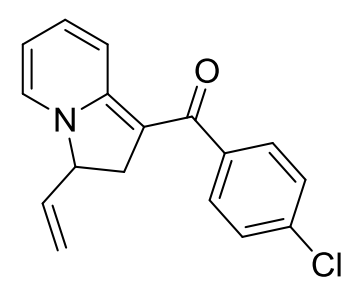

3c, red oil, $90.8 \mathrm{mg}, 80 \%$ yield. ${ }^{1} \mathrm{H}$ NMR (400 MHz, $\left.\mathrm{CDCl}_{3}\right) \delta 8.29(\mathrm{~s}, 1 \mathrm{H}), 7.72-6.53$ (m, 6H), $6.31(\mathrm{~s}, 1 \mathrm{H}), 6.01-5.72(\mathrm{~m}, 1 \mathrm{H}), 5.36(\mathrm{~d}, J=10.0 \mathrm{~Hz}, 1 \mathrm{H}), 5.34(\mathrm{~d}, J=17.6$ $\mathrm{Hz}, 1 \mathrm{H}), 4.96-4.65(\mathrm{~m}, 1 \mathrm{H}), 3.46-3.15(\mathrm{~m}, 1 \mathrm{H}), 2.90(\mathrm{dd}, J=14.0,8.0 \mathrm{~Hz}, 1 \mathrm{H}) .{ }^{13} \mathrm{C}$ NMR $\left(100 \mathrm{MHz}, \mathrm{CDCl}_{3}\right) \delta 182.0,155.3,141.9,138.6,135.5,134.1,128.4,127.8$, 
120.4, 119.8, 117.1, 111.0, 95.9, 68.5, 35.5. IR (thin film): $v_{\max }\left(\mathrm{cm}^{-1}\right)=3082,2919$, 2850, 1624, 1592, 1550, 1466, 1387, 1288, 1166, 1129, 1087, 1012, 836, 761, 522. HRMS-ESI calcd for $\mathrm{C}_{17} \mathrm{H}_{15} \mathrm{ClNO}[\mathrm{M}+\mathrm{H}]^{+}: 284.0837$. Found: 284.0834 .

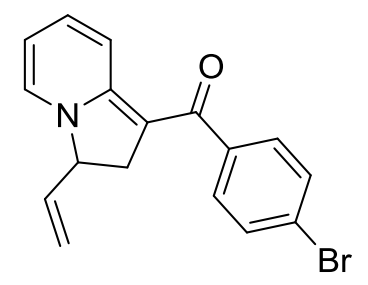

3d, red oil (Known compound, see: Yang, Z.-P.; Wu, Q.-F.; You, S.-L. Angew. Chem. Int. Ed. 2014, 53, 6986). 97.2 mg, 74\% yield. ${ }^{1} \mathrm{H}$ NMR (400 MHz, $\left.\mathrm{CDCl}_{3}\right) \delta 8.31(\mathrm{~s}$, $1 \mathrm{H}), 7.65-7.03(\mathrm{~m}, 6 \mathrm{H}), 6.30(\mathrm{~s}, 1 \mathrm{H}), 6.04-5.80(\mathrm{~m}, 1 \mathrm{H}), 5.39(\mathrm{~d}, J=9.6 \mathrm{~Hz}, 1 \mathrm{H})$, $5.36(\mathrm{~d}, J=14.8 \mathrm{~Hz}, 1 \mathrm{H}), 4.93-4.68(\mathrm{~m}, 1 \mathrm{H}), 3.44-3.19(\mathrm{~m}, 1 \mathrm{H}), 2.92(\mathrm{dd}, J=14.4$, $8.4 \mathrm{~Hz}, 1 \mathrm{H})$.

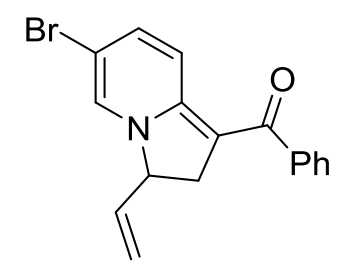

3e, red solid, m.p. $=113.5-114.7^{\circ} \mathrm{C} .68 .3 \mathrm{mg}, 52 \%$ yield. ${ }^{1} \mathrm{H}$ NMR $\left(400 \mathrm{MHz}, \mathrm{CDCl}_{3}\right)$ $\delta$ 9.05-6.58 (m, 8H), 6.01-5.82 (m, 1H), $5.41(\mathrm{~d}, J=8.8 \mathrm{~Hz}, 1 \mathrm{H}), 5.38(\mathrm{~d}, J=15.6 \mathrm{~Hz}$, $1 \mathrm{H}), 4.86-4.70(\mathrm{~m}, 1 \mathrm{H}), 3.40-3.17(\mathrm{~m}, 1 \mathrm{H}), 2.94(\mathrm{dd}, J=14.0,9.6 \mathrm{~Hz}, 1 \mathrm{H}) .{ }^{13} \mathrm{C} \mathrm{NMR}$ $\left(100 \mathrm{MHz}, \mathrm{CDCl}_{3}\right) \delta 191.6,185.7,152.6,143.1,140.0,135.4,134.3,129.1,128.2$, 126.9, 121.1, 120.2, 100.9, 68.3, 35.7. IR (thin film): $v_{\max }\left(\mathrm{cm}^{-1}\right)=3058,3016,2954$, 2920, 2850, 1614, 1583, 1529, 1468, 1414, 1390, 1328, 1297, 1258, 1174, 1146, 1127, 1059, 1026, 998, 969, 950, 864, 838, 787, 724, 692, 657, 621, 528, 474, 417. HRMSESI calcd for $\mathrm{C}_{17} \mathrm{H}_{15} \mathrm{BrNO}[\mathrm{M}+\mathrm{H}]^{+}$: 328.0332. Found: 328.0326 .

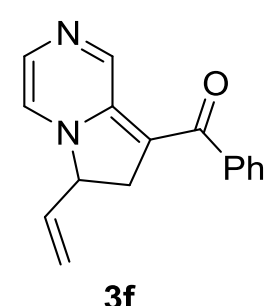

$3 f$<smiles>C=CC1CC(c2cnccn2)=C(c2ccccc2)O1</smiles>

3f: $\mathbf{3} \mathbf{f}^{\prime}=12.5: 1$ 
3f, dark solid, m.p. $=120.6-121.4{ }^{\circ} \mathrm{C}$ (Known compound, see: Yang, Z.-P.; Wu, Q.-F.; You, S.-L. Angew. Chem. Int. Ed. 2014, 53, 6986). $58.2 \mathrm{mg}, 58 \%$ yield. ${ }^{1} \mathrm{H}$ NMR (400 $\left.\mathrm{MHz}, \mathrm{CDCl}_{3}\right) \delta 8.43(\mathrm{~s}, 1 \mathrm{H}), 7.54(\mathrm{~d}, J=5.6 \mathrm{~Hz}, 1 \mathrm{H}), 7.53(\mathrm{~d}, J=7.6 \mathrm{~Hz}, 1 \mathrm{H}), 7.44-$ $7.37(\mathrm{~m}, 3 \mathrm{H}), 7.09$ (d, $J=4.4 \mathrm{~Hz}, 1 \mathrm{H}), 6.82$ (d, $J=4.0 \mathrm{~Hz}, 1 \mathrm{H}), 5.91$ (ddd, $J=16.4$, 9.6, $8.8 \mathrm{~Hz}, 1 \mathrm{H}), 5.42(\mathrm{~d}, J=9.6 \mathrm{~Hz}, 1 \mathrm{H}), 5.39(\mathrm{~d}, J=16.4 \mathrm{~Hz}, 1 \mathrm{H}), 4.82-4.67(\mathrm{~m}$, $1 \mathrm{H}), 3.35(\mathrm{dd}, J=15.2,11.6 \mathrm{~Hz}, 1 \mathrm{H}), 2.95(\mathrm{dd}, J=15.6,9.6 \mathrm{~Hz}, 1 \mathrm{H})$.

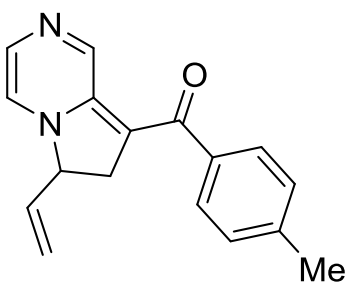

$3 g$

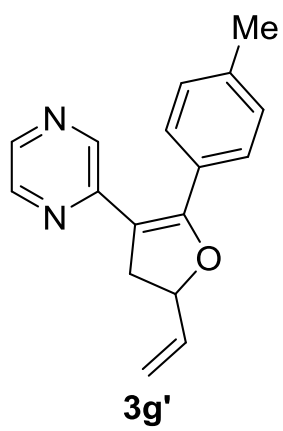

$\mathbf{3 g}: \mathbf{3} \mathbf{g}^{\prime}=5.0: 1$

3g, dark solid, m.p. $=128.7-129.5^{\circ} \mathrm{C}$ (Known compound, see: Yang, Z.-P.; Wu, Q.-F.; Shao, W.; You, S.-L. J. Am. Chem. Soc. 2015, 137, 15899). $54.3 \mathrm{mg}, 51 \%$ yield. ${ }^{1} \mathrm{H}$ NMR (400 MHz, $\left.\mathrm{CDCl}_{3}\right) \delta 8.46(\mathrm{~s}, 1 \mathrm{H}), 7.45(\mathrm{~d}, J=7.2 \mathrm{~Hz}, 2 \mathrm{H}), 7.20$ (d, $J=7.6 \mathrm{~Hz}$, 2H), $7.06(\mathrm{~d}, J=4.0 \mathrm{~Hz}, 1 \mathrm{H}), 6.85-6.70(\mathrm{~m}, 1 \mathrm{H}), 5.99-5.82(\mathrm{~m}, 1 \mathrm{H}), 5.42(\mathrm{~d}, J=9.6$ $\mathrm{Hz}, 1 \mathrm{H}), 5.38(\mathrm{~d}, J=16.4 \mathrm{~Hz}, 1 \mathrm{H}), 4.78-4.66(\mathrm{~m}, 1 \mathrm{H}), 3.36(\mathrm{dd}, J=15.2,12.0 \mathrm{~Hz}$, $1 \mathrm{H}), 2.95(\mathrm{dd}, J=15.6,10.0 \mathrm{~Hz}, 1 \mathrm{H}), 2.38(\mathrm{~s}, 3 \mathrm{H})$.

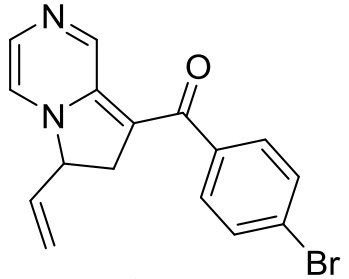

3h

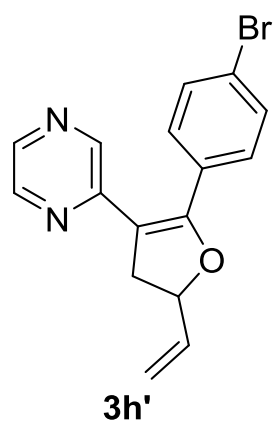

$3 h: 3 h^{\prime}=4.5: 1$

3h, dark solid, m.p. $=129.1-129.8{ }^{\circ} \mathrm{C}$ (Known compound, see: Yang, Z.-P.; Wu, Q.F.; Shao, W.; You, S.-L. J. Am. Chem. Soc. 2015, 137, 15899). 48.7 mg, $37 \%$ yield. ${ }^{1} \mathrm{H}$ NMR (400 MHz, $\left.\mathrm{CDCl}_{3}\right) \delta 8.65(\mathrm{~s}, 1 \mathrm{H}), 7.53(\mathrm{~d}, J=8.4 \mathrm{~Hz}, 2 \mathrm{H}), 7.43(\mathrm{~d}, J=8.4$ $\mathrm{Hz}, 2 \mathrm{H}), 7.17(\mathrm{~d}, J=4.0 \mathrm{~Hz}, 1 \mathrm{H}), 6.86(\mathrm{~d}, J=3.2 \mathrm{~Hz}, 1 \mathrm{H}), 5.92(\mathrm{ddd}, J=17.2,10.0$, $8.8 \mathrm{~Hz}, 1 \mathrm{H}), 5.44(\mathrm{~d}, J=10.8 \mathrm{~Hz}, 1 \mathrm{H}), 5.41(\mathrm{~d}, J=18.0 \mathrm{~Hz}, 1 \mathrm{H}), 4.83-4.70(\mathrm{~m}, 1 \mathrm{H})$, $3.34(\mathrm{dd}, J=15.2,11.6 \mathrm{~Hz}, 1 \mathrm{H}), 2.94(\mathrm{dd}, J=15.2,9.6 \mathrm{~Hz}, 1 \mathrm{H})$. 
3h', brown solid, m.p. $=61.7-62.5{ }^{\circ} \mathrm{C} .10 .5 \mathrm{mg}, 8 \%$ yield. ${ }^{1} \mathrm{H}$ NMR $(400 \mathrm{MHz}$, $\left.\mathrm{CDCl}_{3}\right) \delta 8.42(\mathrm{t}, J=3.2,1.6,1.6 \mathrm{~Hz}, 1 \mathrm{H}), 8.38-8.33(\mathrm{~m}, 1 \mathrm{H}), 8.22(\mathrm{~d}, J=2.4 \mathrm{~Hz}$, 1H), $7.50(\mathrm{~d}, J=8.4 \mathrm{~Hz}, 2 \mathrm{H}), 7.43(\mathrm{~d}, J=8.4 \mathrm{~Hz}, 2 \mathrm{H}), 6.09$ (ddd, $J=17.2,10.4,6.8$ $\mathrm{Hz}, 1 \mathrm{H}), 5.42$ (d, $J=17.2 \mathrm{~Hz}, 1 \mathrm{H}), 5.28(\mathrm{~d}, J=10.0 \mathrm{~Hz}, 1 \mathrm{H}), 5.26-5.20(\mathrm{~m}, 1 \mathrm{H}), 3.46$ $(\mathrm{dd}, J=14.8,10.0 \mathrm{~Hz}, 1 \mathrm{H}), 3.14(\mathrm{dd}, J=15.2,8.4 \mathrm{~Hz}, 1 \mathrm{H}) .{ }^{13} \mathrm{C}$ NMR $(100 \mathrm{MHz}$, $\left.\mathrm{CDCl}_{3}\right) \delta 155.0,150.9,143.8,143.1,140.6,137.2,131.9,130.1,130.0,124.2,117.1$, 108.6, 81.5, 39.4. IR (thin film): $v_{\max }\left(\mathrm{cm}^{-1}\right)=3645,3357,3063,2993,2920,2853$, 2383, 2322, 2289, 2237, 2180, 2101, 1992, 1913, 1719, 1633, 1583, 1512, 1482, 1403, 1356, 1233, 1150, 1099, 1067, 1003, 916, 829, 728, 650, 623, 528, 473. HRMS-ESI calcd for $\mathrm{C}_{16} \mathrm{H}_{14} \mathrm{BrN}_{2} \mathrm{O}[\mathrm{M}+\mathrm{H}]^{+}: 329.0284$. Found: 329.0278.

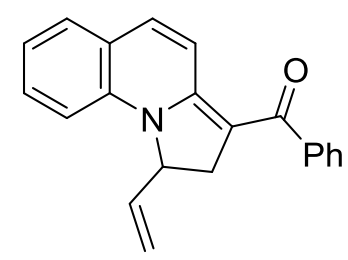

3i, yellow solid, m.p. $=132.5-133.2{ }^{\circ} \mathrm{C}$ (Known compound, see: Yang, Z.-P.; Wu, Q.F.; Shao, W.; You, S.-L. J. Am. Chem. Soc. 2015, 137, 15899). 104.3 mg, $87 \%$ yield. ${ }^{1} \mathrm{H}$ NMR $\left(400 \mathrm{MHz}, \mathrm{CDCl}_{3}\right) \delta$ 7.60-7.50 (m, 2H), 7.46-7.16 (m, 7H), 7.10-7.00 (m, 2H), 5.91 (ddd, $J=17.2,10.0,7.2 \mathrm{~Hz}, 1 \mathrm{H}), 5.17$ (d, $J=10.4 \mathrm{~Hz}, 1 \mathrm{H}), 5.12$ (d, $J=$ $17.5 \mathrm{~Hz}, 1 \mathrm{H}), 5.13-5.04(\mathrm{~m}, 1 \mathrm{H}), 3.54(\mathrm{dd}, J=14.8,11.6 \mathrm{~Hz}, 1 \mathrm{H}), 2.91$ (dd, $J=15.2$, $2.4 \mathrm{~Hz}, 1 \mathrm{H})$.

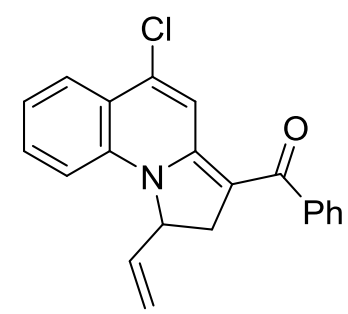

3j, red solid, m.p. $=96.2-97.1{ }^{\circ} \mathrm{C} .79 .7 \mathrm{mg}, 60 \%$ yield. ${ }^{1} \mathrm{H}$ NMR $\left(400 \mathrm{MHz}, \mathrm{CDCl}_{3}\right) \delta$ $7.77(\mathrm{~d}, J=8.0 \mathrm{~Hz}, 1 \mathrm{H}), 7.68(\mathrm{~s}, 1 \mathrm{H}), 7.61-7.51(\mathrm{~m}, 2 \mathrm{H}), 7.49-7.36(\mathrm{~m}, 4 \mathrm{H}), 7.12(\mathrm{t}, J$ $=7.6 \mathrm{~Hz}, 1 \mathrm{H}), 7.04(\mathrm{~d}, J=8.4 \mathrm{~Hz}, 1 \mathrm{H}), 5.90(\mathrm{ddd}, J=17.2,10.4,6.8 \mathrm{~Hz}, 1 \mathrm{H}), 5.19$ (d, $J=10.4 \mathrm{~Hz}, 1 \mathrm{H}), 5.14(\mathrm{~d}, J=17.2 \mathrm{~Hz}, 1 \mathrm{H}), 5.11-5.06(\mathrm{~m}, 1 \mathrm{H}), 3.55(\mathrm{dd}, J=15.2$, $11.6 \mathrm{~Hz}, 1 \mathrm{H}), 2.89(\mathrm{dd}, J=15.2,3.2 \mathrm{~Hz}, 1 \mathrm{H}) .{ }^{13} \mathrm{C} \mathrm{NMR}\left(100 \mathrm{MHz}, \mathrm{CDCl}_{3}\right) \delta 188.7$, $151.4,142.7,142.1,138.5,135.8,132.1,129.8,128.4,127.2,125.9,122.3,121.5$, 
118.8, 116.6, 114.3, 102.6, 62.9, 36.8. IR (thin film): $v_{\max }\left(\mathrm{cm}^{-1}\right)=3057,2921,2851$, 2326, 2118, 2086, 1918, 1742, 1678, 1612, 1598, 1547, 1508, 1467, 1442, 1390, 1318, 1245, 1169, 1140, 1072, 998, 950, 925, 883, 844, 753, 717, 697. HRMS-ESI calcd for $\mathrm{C}_{21} \mathrm{H}_{17} \mathrm{ClNO}[\mathrm{M}+\mathrm{H}]^{+}$: 334.0993. Found: 334.0987.

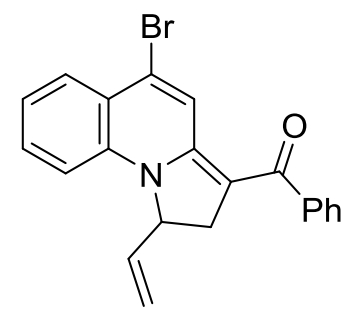

3k, red solid, m.p. $=85.8-86.1{ }^{\circ} \mathrm{C} .63 .2 \mathrm{mg}, 42 \%$ yield. ${ }^{1} \mathrm{H} \mathrm{NMR}\left(400 \mathrm{MHz}, \mathrm{CDCl}_{3}\right) \delta$ $7.92(\mathrm{~s}, 1 \mathrm{H}), 7.75(\mathrm{~d}, J=8.0 \mathrm{~Hz}, 1 \mathrm{H}), 7.61-7.51(\mathrm{~m}, 2 \mathrm{H}), 7.49-7.36(\mathrm{~m}, 4 \mathrm{H}), 7.11(\mathrm{t}, J$ $=7.2 \mathrm{~Hz}, 1 \mathrm{H}), 7.01(\mathrm{~d}, J=8.4 \mathrm{~Hz}, 1 \mathrm{H}), 5.89(\mathrm{ddd}, J=17.2,10.0,7.6 \mathrm{~Hz}, 1 \mathrm{H}), 5.19(\mathrm{~d}$, $J=10.8 \mathrm{~Hz}, 1 \mathrm{H}), 5.14(\mathrm{~d}, J=18.0 \mathrm{~Hz}, 1 \mathrm{H}), 5.10-5.02(\mathrm{~m}, 1 \mathrm{H}), 3.54(\mathrm{dd}, J=14.8$, $12.0 \mathrm{~Hz}, 1 \mathrm{H}), 2.93-2.80(\mathrm{~m}, 1 \mathrm{H}) .{ }^{13} \mathrm{C} \mathrm{NMR}\left(100 \mathrm{MHz}, \mathrm{CDCl}_{3}\right) \delta 188.8,151.4,142.7$, 138.3, 135.8, 133.8, 132.1, 129.8, 128.6, 128.3, 127.2, 122.8, 122.5, 122.4, 116.6, 114.2, 102.4, 62.9, 36.7. IR (thin film): $v_{\max }\left(\mathrm{cm}^{-1}\right)=3079,3056,2921,2851,2653$, 2331, 2308, 2118, 2090, 2068, 1994, 1743, 1711, 1679, 1608, 1581, 1543, 1505, 1467, 1439, 1387, 1316, 1285, 1266, 1245, 1170, 1096, 1072, 1045, 1024, 998, 969, 949, 922, 903, 885, 845, 789, 748, 716, 699, 656, 614. HRMS-ESI calcd for $\mathrm{C}_{21} \mathrm{H}_{17} \mathrm{BrNO}$ $[\mathrm{M}+\mathrm{H}]^{+}:$378.0488. Found: 378.0477 .

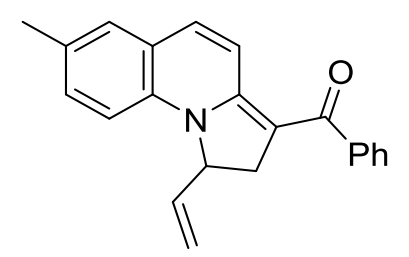

31, red oil, $98.6 \mathrm{mg}, 79 \%$ yield. ${ }^{1} \mathrm{H}$ NMR (400 $\left.\mathrm{MHz}, \mathrm{CDCl}_{3}\right) \delta 7.59-7.51(\mathrm{~m}, 2 \mathrm{H})$, 7.50-7.25 (m, 4H), 7.19 (d, $J=8.8 \mathrm{~Hz}, 2 \mathrm{H}), 7.15$ (s, 1H), 6.97 (d, $J=8.4 \mathrm{~Hz}, 1 \mathrm{H})$, $5.89(\mathrm{ddd}, J=17.2,10.0,6.8 \mathrm{~Hz}, 1 \mathrm{H}), 5.15(\mathrm{~d}, J=10.4 \mathrm{~Hz}, 1 \mathrm{H}), 5.10(\mathrm{~d}, J=17.6 \mathrm{~Hz}$, 1H), 5.11-5.04 (m, 1H), $3.53(\mathrm{dd}, J=14.8,11.6 \mathrm{~Hz}, 1 \mathrm{H}), 2.90(\mathrm{dd}, J=15.2,2.8 \mathrm{~Hz}$, 1H), $2.32(\mathrm{~s}, 3 \mathrm{H}) .{ }^{13} \mathrm{C} \mathrm{NMR}\left(100 \mathrm{MHz}, \mathrm{CDCl}_{3}\right) \delta 188.0,152.7,143.2,136.1,136.0$, 132.2, 131.6, 129.7, 129.2, 128.2, 128.1, 127.1, 123.3, 118.8, 116.1, 113.9, 102.1, 62.7, 36.4, 20.6. IR (thin film): $v_{\max }\left(\mathrm{cm}^{-1}\right)=3667,3055,3023,2920,2854,2220$, 1614, 1581, 1565, 1505, 1476, 1414, 1369, 1343, 1293, 1266, 1235, 1170, 1118, 1070, 
1055, 1026, 997, 945, 921, 884, 826, 805, 787, 753, 718, 696, 664, 643. HRMS-ESI calcd for $\mathrm{C}_{22} \mathrm{H}_{20} \mathrm{NO}[\mathrm{M}+\mathrm{H}]^{+}: 314.1539$. Found: 314.1531 .

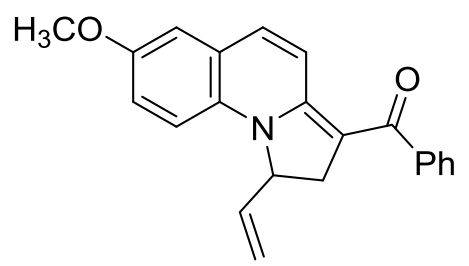

3m, red solid, m.p. $=118.3-118.9{ }^{\circ} \mathrm{C} .112 .9 \mathrm{mg}, 86 \%$ yield. ${ }^{1} \mathrm{H}$ NMR $(400 \mathrm{MHz}$, $\left.\mathrm{CDCl}_{3}\right) \delta 7.60-7.52(\mathrm{~m}, 2 \mathrm{H}), 7.51-7.26(\mathrm{~m}, 4 \mathrm{H}), 7.23(\mathrm{~d}, J=8.8 \mathrm{~Hz}, 1 \mathrm{H}), 7.08-6.98$ $(\mathrm{m}, 2 \mathrm{H}), 6.84(\mathrm{~s}, 1 \mathrm{H}), 5.88(\mathrm{ddd}, J=17.6,10.4,7.2 \mathrm{~Hz}, 1 \mathrm{H}), \delta 5.14(\mathrm{~d}, J=10.4 \mathrm{~Hz}$, $1 \mathrm{H}), 5.09(\mathrm{~d}, J=17.2 \mathrm{~Hz}, 1 \mathrm{H}), 5.10-5.03(\mathrm{~m}, 1 \mathrm{H}), 3.78(\mathrm{~s}, 3 \mathrm{H}), 3.53(\mathrm{dd}, J=14.8$, $11.6 \mathrm{~Hz}, 1 \mathrm{H}), 2.89(\mathrm{dd}, J=14.8,2.8 \mathrm{~Hz}, 1 \mathrm{H}) .{ }^{13} \mathrm{C} \mathrm{NMR}\left(100 \mathrm{MHz}, \mathrm{CDCl}_{3}\right) \delta 187.4$, $154.7,152.5,143.3,136.1,136.0,132.6,129.1,128.1,127.1,124.1,119.6,119.5$, 116.1, 115.3, 110.1, 101.5, 63.0, 55.6, 36.4. IR (thin film): $v_{\max }\left(\mathrm{cm}^{-1}\right)=3360,3055$, 3008, 2921, 2851, 2325, 2117, 2088, 1997, 1892, 1864, 1708, 1612, 1563, 1505, 1476, 1442, 1412, 1340, 1284, 1239, 1190, 1172, 1127, 1070, 1053, 1026, 997, 947, 922, $865,800,788,751,717,697,665$. HRMS-ESI calcd for $\mathrm{C}_{22} \mathrm{H}_{20} \mathrm{NO}_{2}[\mathrm{M}+\mathrm{H}]^{+}$: 330.1489. Found: 330.1482 .

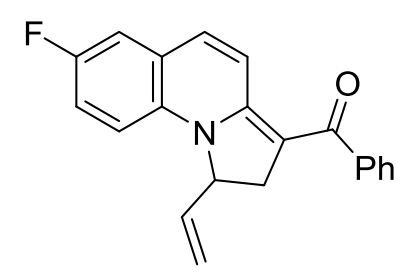

3n, red solid, m.p. $=67.2-67.9{ }^{\circ} \mathrm{C} .92 .3 \mathrm{mg}, 73 \%$ yield. ${ }^{1} \mathrm{H} \mathrm{NMR}\left(400 \mathrm{MHz}, \mathrm{CDCl}_{3}\right) \delta$ $7.55(\mathrm{~d}, J=5.2 \mathrm{~Hz}, 1 \mathrm{H}), 7.54(\mathrm{~d}, J=7.2 \mathrm{~Hz}, 1 \mathrm{H}), 7.51-7.22(\mathrm{~m}, 4 \mathrm{H}), 7.16(\mathrm{~d}, J=9.6$ $\mathrm{Hz}, 1 \mathrm{H}), 7.10(\mathrm{td}, J=8.6,2.8 \mathrm{~Hz}, 1 \mathrm{H}), 7.07-6.97$ (m, 2H), 5.90 (ddd, $J=17.2,10.4$, $6.8 \mathrm{~Hz}, 1 \mathrm{H}), 5.18$ (d, $J=10.4 \mathrm{~Hz}, 1 \mathrm{H}), 5.13$ (d, $J=17.2 \mathrm{~Hz}, 1 \mathrm{H}), 5.09-5.03$ (m, 1H), $3.55(\mathrm{dd}, J=15.2,11.6 \mathrm{~Hz}, 1 \mathrm{H}), 2.91(\mathrm{dd}, J=15.2,3.2 \mathrm{~Hz}, 1 \mathrm{H}) .{ }^{19} \mathrm{~F}$ NMR $(376 \mathrm{MHz}$, $\left.\mathrm{CDCl}_{3}\right) \delta-120.5(\mathrm{~m}) .{ }^{13} \mathrm{C} \mathrm{NMR}\left(100 \mathrm{MHz}, \mathrm{CDCl}_{3}\right) \delta 188.6,157.6(\mathrm{~d}, J=242.6 \mathrm{~Hz})$ 152.3, 143.0, 135.9, 135.2 (d, $J=1.9 \mathrm{~Hz}), 134.8(\mathrm{~d}, J=1.2 \mathrm{~Hz}), 129.6,128.3,127.2$, $124.2(\mathrm{~d}, J=8.6 \mathrm{~Hz}), 120.4,118.6(\mathrm{~d}, J=24.0 \mathrm{~Hz}), 116.4,115.4(\mathrm{~d}, J=8.0 \mathrm{~Hz})$, $113.4(\mathrm{~d}, J=22.6 \mathrm{~Hz}), 102.9,63.0,36.6$. IR (thin film): $v_{\max }\left(\mathrm{cm}^{-1}\right)=3057,2920$, 
2853, 2120, 2080, 1706, 1622, 1566, 1510, 1478, 1421, 1370, 1342, 1287, 1239, 1155, $1127,1071,1055,1025,998,937,872,845,805,787,750,713,666,623$. HRMSESI calcd for $\mathrm{C}_{21} \mathrm{H}_{17} \mathrm{FNO}[\mathrm{M}+\mathrm{H}]^{+}$: 318.1289. Found: 318.1281 .

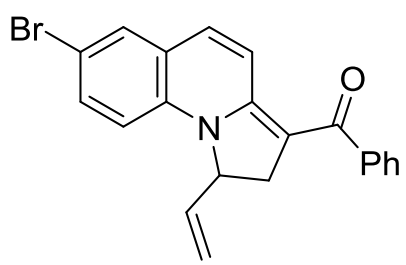

3o, red solid, m.p. $=83.1-83.6{ }^{\circ} \mathrm{C} .125 .9 \mathrm{mg}, 83 \%$ yield. ${ }^{1} \mathrm{H} \mathrm{NMR}\left(400 \mathrm{MHz}, \mathrm{CDCl}_{3}\right)$ $\delta$ 7.59-7.49 (m, 2H), 7.46-7.34 (m, 5H), 7.29 (s, 1H), 7.06 (d, J=10.0 Hz, 1H), 6.87 $(\mathrm{d}, J=8.8 \mathrm{~Hz}, 1 \mathrm{H}), 5.87$ (ddd, $J=17.2,10.4,6.8 \mathrm{~Hz}, 1 \mathrm{H}), 5.17$ (d, $J=10.4 \mathrm{~Hz}, 1 \mathrm{H})$, $5.10(\mathrm{~d}, J=17.2 \mathrm{~Hz}, 1 \mathrm{H}), 5.02(\mathrm{ddd}, J=10.4,6.8,3.2 \mathrm{~Hz}, 1 \mathrm{H}), 3.52(\mathrm{dd}, J=15.2$, $11.6 \mathrm{~Hz}, 1 \mathrm{H}), 2.88(\mathrm{dd}, J=15.2,3.2 \mathrm{~Hz}, 1 \mathrm{H}) .{ }^{13} \mathrm{C} \mathrm{NMR}\left(100 \mathrm{MHz}, \mathrm{CDCl}_{3}\right) \delta 188.9$, $151.8,142.7,137.1,135.6,134.5,133.5,130.2$, 129.6, 128.2, 127.1, 124.7, 120.1, 116.4, 115.4, 114.2, 103.5, 62.6, 36.6. IR (thin film): $v_{\max }\left(\mathrm{cm}^{-1}\right)=3054,2919,2850$, 2100, 1678, 1620, 1582, 1503, 1469, 1410, 1366, 1287, 1259, 1202, 1175, 1157, 1133, 1075, 1055, 1024, 997, 943, 879, 804, 787, 749, 698, 664. HRMS-ESI calcd for $\mathrm{C}_{21} \mathrm{H}_{17} \mathrm{BrNO}[\mathrm{M}+\mathrm{H}]^{+}:$378.0488. Found: 378.0479 .

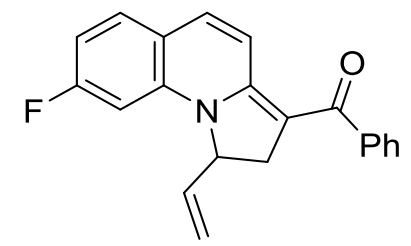

3p, red solid, m.p. = 115.9-116.4 ${ }^{\circ} \mathrm{C} .101 .6 \mathrm{mg}, 80 \%$ yield. ${ }^{1} \mathrm{H}$ NMR $(400 \mathrm{MHz}$, $\left.\mathrm{CDCl}_{3}\right) \delta$ 7.61-7.48 (m, 2H), 7.47-7.31 (m, 3H), 7.31-7.20 (m, 2H), $7.15(\mathrm{~d}, J=9.6$ $\mathrm{Hz}, 1 \mathrm{H}), 6.80-6.67$ (m, 2H), 5.89 (ddd, $J=17.6,10.4,7.2 \mathrm{~Hz}, 1 \mathrm{H}), 5.20$ (d, $J=10.0$ $\mathrm{Hz}, 1 \mathrm{H}), 5.15(\mathrm{~d}, J=17.2 \mathrm{~Hz}, 1 \mathrm{H}), 4.99$ (ddd, $J=10.8,6.8,3.2 \mathrm{~Hz}, 1 \mathrm{H}), 3.54(\mathrm{dd}, J=$ 15.2, $11.6 \mathrm{~Hz}, 1 \mathrm{H}), 2.89(\mathrm{dd}, J=15.2,3.2 \mathrm{~Hz}, 1 \mathrm{H}) .{ }^{19} \mathrm{~F} \mathrm{NMR}\left(376 \mathrm{MHz}, \mathrm{CDCl}_{3}\right) \delta-$ $106.2(\mathrm{~m}) .{ }^{13} \mathrm{C}$ NMR $\left(100 \mathrm{MHz}, \mathrm{CDCl}_{3}\right) \delta 189.0,164.1(\mathrm{~d}, J=250.1 \mathrm{~Hz}), 152.2$, 142.8, 139.7 (d, $J=12.0 \mathrm{~Hz}), 135.5,135.3,130.0(\mathrm{~d}, J=10.4 \mathrm{~Hz}), 129.6,128.2$, 127.2, $119.8(\mathrm{~d}, J=2 \mathrm{~Hz}), 117.9(\mathrm{~d}, J=2.6 \mathrm{~Hz}), 116.6,109.7(\mathrm{~d}, J=23.1 \mathrm{~Hz}), 103.3$, $100.8(\mathrm{~d}, J=27.0 \mathrm{~Hz}), 62.8,36.6$. IR (thin film): $v_{\max }\left(\mathrm{cm}^{-1}\right)=3082,3058,3038,2956$, 2910, 2851, 2324, 2267, 2219, 2114, 2088, 1996, 1951, 1906, 1861, 1828, 1810, 1715 , 
$1624,1570,1518,1472,1411,1373,1332,1293,1251,1219,1192,1164,1140,1123$, 1089, 1057, 1024, 990, 922, 897, 857, 830, 784, 718, 698, 669, 645. HRMS-ESI calcd for $\mathrm{C}_{21} \mathrm{H}_{17} \mathrm{FNO}[\mathrm{M}+\mathrm{H}]^{+}:$318.1289. Found: 318.1281 .<smiles>C=CC1CC(C(=O)c2ccccc2)=C2C=Cc3ccc(Cl)cc3N21</smiles>

3q, red solid, m.p. $=98.5-99.7{ }^{\circ} \mathrm{C} .110 .8 \mathrm{mg}, 83 \%$ yield. ${ }^{1} \mathrm{H}$ NMR $\left(400 \mathrm{MHz}, \mathrm{CDCl}_{3}\right)$ $\delta$ 7.58-7.50 (m, 2H), 7.44-7.34 (m, 3H), 7.32-7.17 (m, 2H), $7.12(\mathrm{~d}, J=10.0 \mathrm{~Hz}, 1 \mathrm{H})$, $6.97(\mathrm{~d}, J=10.4 \mathrm{~Hz}, 2 \mathrm{H}), 5.88(\mathrm{ddd}, J=17.2,10.0,6.8 \mathrm{~Hz}, 1 \mathrm{H}), 5.20(\mathrm{~d}, J=10.4 \mathrm{~Hz}$, $1 \mathrm{H}), 5.14(\mathrm{~d}, J=17.2 \mathrm{~Hz}, 1 \mathrm{H}), 5.00(\mathrm{ddd}, J=10.8,6.8,3.2 \mathrm{~Hz}, 1 \mathrm{H}), 3.53(\mathrm{dd}, J=$ 15.2, $11.6 \mathrm{~Hz}, 1 \mathrm{H}), 2.88(\mathrm{dd}, J=15.2,3.2 \mathrm{~Hz}, 1 \mathrm{H}) .{ }^{13} \mathrm{C} \mathrm{NMR}\left(100 \mathrm{MHz}, \mathrm{CDCl}_{3}\right) \delta$ $189.1,151.9,142.8,139.0,136.7,135.5,135.1,129.7,129.1,128.3,127.2,122.0$, 121.7, 119.1, 116.6, 113.7, 103.5, 62.5, 36.6. IR (thin film): $v_{\max }\left(\mathrm{cm}^{-1}\right)=3668,3058$, 2921, 2852, 2225, 2122, 2093, 1708, 1620, 1581, 1508, 1466, 1404, 1373, 1320, 1277 , 1250, 1213, 1175, 1158, 1131, 1092, 1057, 1025, 999, 965, 921, 877, 833, 785, 749, 701, 669, 644, 619. HRMS-ESI calcd for $\mathrm{C}_{21} \mathrm{H}_{17} \mathrm{ClNO}[\mathrm{M}+\mathrm{H}]^{+}$: 334.0993. Found: 334.0987.

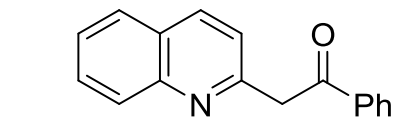

$1 \mathrm{i}$

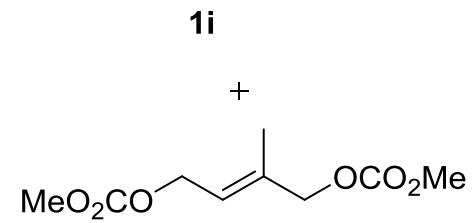

2c

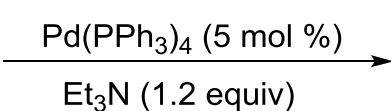

THF, $25^{\circ} \mathrm{C}$

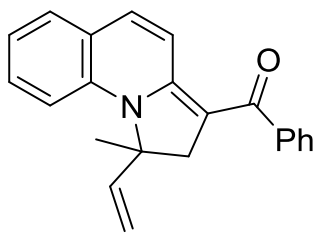

$3 r$ $+$<smiles>C=CC1(C)CC(c2ccc3ccccc3n2)=C(c2ccccc2)O1</smiles> 


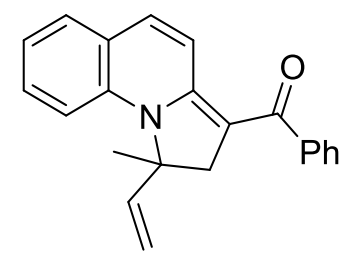

3r, red oil. $55.2 \mathrm{mg}, 44 \%$ yield. ${ }^{1} \mathrm{H}$ NMR $\left(400 \mathrm{MHz}, \mathrm{CDCl}_{3}\right) \delta$ 7.62-7.46 (m, 4H), 7.43-7.35 (m, 3H), $7.31(\mathrm{~m}, 2 \mathrm{H}), 7.21(\mathrm{~d}, J=9.2 \mathrm{~Hz}, 1 \mathrm{H}), 7.02(\mathrm{t}, J=7.2 \mathrm{~Hz}, 1 \mathrm{H})$, $6.37(\mathrm{dd}, J=17.2,10.4 \mathrm{~Hz}, 1 \mathrm{H}), 5.32$ (d, $J=14.4 \mathrm{~Hz}, 1 \mathrm{H}), 5.29$ (d, $J=7.6 \mathrm{~Hz}, 1 \mathrm{H})$, $3.25(\mathrm{~d}, J=14.8 \mathrm{~Hz}, 1 \mathrm{H}), 2.97(\mathrm{~d}, J=15.2 \mathrm{~Hz}, 1 \mathrm{H}), 1.72(\mathrm{~s}, 3 \mathrm{H}) .{ }^{13} \mathrm{C}$ NMR $(100$ $\left.\mathrm{MHz}, \mathrm{CDCl}_{3}\right) \delta 188.6,152.6,143.3,143.2,137.9,136.2,130.3,129.3,128.6,128.1$, $127.1,124.1,121.8,119.1,114.4,113.6,101.4,70.1,46.9,22.1$. IR (thin film): $v_{\max }\left(\mathrm{cm}^{-1}\right)=3393,3189,3058,2920,2851,2437,2383,2321,2120,1997,1912$, 1703, 1617, 1512, 1466, 1415, 1372, 1249, 1221, 1126, 1068, 1024, 992, 919, 872, $828,784,755,711,658,613,511,434$. HRMS-ESI calcd for $\mathrm{C}_{22} \mathrm{H}_{20} \mathrm{NO}[\mathrm{M}+\mathrm{H}]^{+}$: 314.1539. Found: 314.1532.

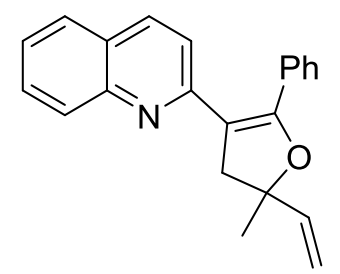

3s, yellow solid, m.p. $=67.6-68.1{ }^{\circ} \mathrm{C} .38 .9 \mathrm{mg}, 31 \%$ yield. ${ }^{1} \mathrm{H}$ NMR $(400 \mathrm{MHz}$, $\left.\mathrm{CDCl}_{3}\right) \delta 7.95(\mathrm{~d}, J=8.4 \mathrm{~Hz}, 1 \mathrm{H}), 7.71(\mathrm{~d}, J=8.8 \mathrm{~Hz}, 1 \mathrm{H}), 7.66-7.55(\mathrm{~m}, 4 \mathrm{H}), 7.42-$ $7.30(\mathrm{~m}, 4 \mathrm{H}), 7.17(\mathrm{~d}, J=8.8 \mathrm{~Hz}, 1 \mathrm{H}), 6.15(\mathrm{dd}, J=17.6,10.4 \mathrm{~Hz}, 1 \mathrm{H}), 5.40$ (d, $J=$ $18.0 \mathrm{~Hz}, 1 \mathrm{H}), 5.14(\mathrm{~d}, J=10.8 \mathrm{~Hz}, 1 \mathrm{H}), 3.48(\mathrm{~d}, J=15.2 \mathrm{~Hz}, 1 \mathrm{H}), 3.39$ (d, $J=15.2$ $\mathrm{Hz}, 1 \mathrm{H}), 1.64(\mathrm{~s}, 3 \mathrm{H}) .{ }^{13} \mathrm{C} \mathrm{NMR}\left(100 \mathrm{MHz}, \mathrm{CDCl}_{3}\right) \delta 155.6,155.0,148.3,142.2$, 134.6, 132.0, 129.7, 129.3, 128.90, 128.88, 128.5, 127.4, 126.3, 125.5, 120.8, 112.6, 111.5, 85.2, 46.1, 26.5. IR (thin film): $v_{\max }\left(\mathrm{cm}^{-1}\right)=3875,3772,3734,3695,3657$, 3447, 3388, 3052, 2973, 2909, 2850, 2652, 2435, 2386, 2342, 2246, 2212, 2098, 1994, 1946, 1914, 1885, 1840, 1811, 1768, 1722, 1631, 1597, 1549, 1496, 1448, $1417,1363,1330,1295,1234,1180,1110,1067,1027,981,926,876,826,796,756$, $692,648,597,517,470$. HRMS-ESI calcd for $\mathrm{C}_{22} \mathrm{H}_{20} \mathrm{NO}[\mathrm{M}+\mathrm{H}]^{+}:$314.1539. Found: 314.1531 . 


\section{Procedure for the synthesis of intermediate S4}

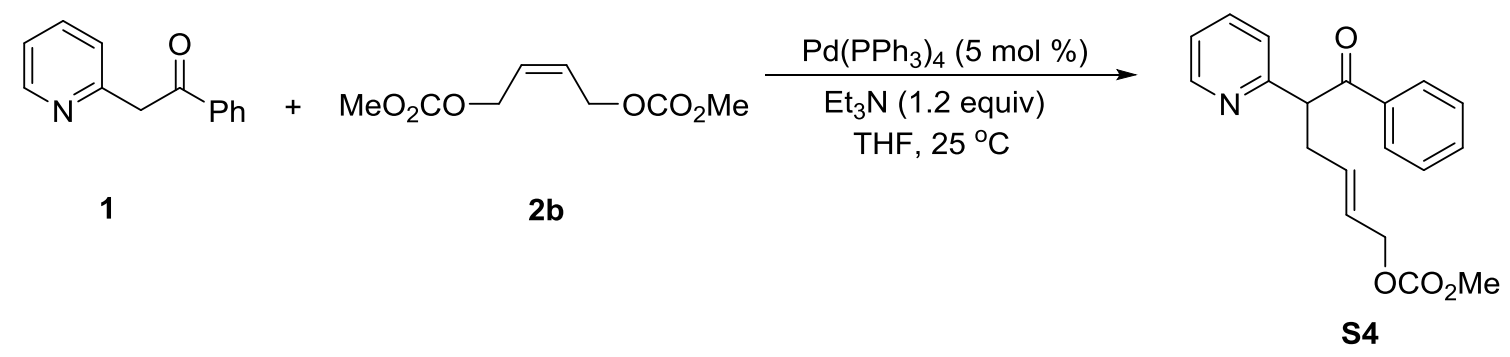

A flame-dried Schlenk tube was cooled to room temperature and filled with argon. To this flask were added 1 ( $0.4 \mathrm{mmol}, 1.0$ equiv), allylic carbonate $\mathbf{2 b}$ (0.48 mmol, 1.2 equiv), $\mathrm{Pd}\left(\mathrm{PPh}_{3}\right)_{4}(0.02 \mathrm{mmol}, 5 \mathrm{~mol} \%), \mathrm{Et}_{3} \mathrm{~N}(0.48 \mathrm{mmol}, 1.2$ equiv) and THF (4 $\mathrm{mL})$. The reaction mixture was stirred at room temperature for $2 \mathrm{~h}$. After the generation of intermediate (monitored by TLC), brine $(20 \mathrm{~mL})$ was added to quench the reaction. This mixture was extracted with EtOAc $(3 \times 15 \mathrm{~mL})$. The extracts were combined, dried over $\mathrm{Na}_{2} \mathrm{SO}_{4}$, filtered and evaporated under reduced pressure. Then the residue was purified by silica gel column chromatography $(\mathrm{PE} / \mathrm{EtOAc}=5 / 1)$ to afford S4.<smiles>COC/C=C/CC(C(=O)c1ccccc1)c1ccccn1</smiles>

S4, yellow oil (Known compound, see: Yang, Z.-P.; Wu, Q.-F.; You, S.-L. Angew. Chem. Int. Ed. 2014, 53, 6986). $75.5 \mathrm{mg}, 58 \%$ yield. ${ }^{1} \mathrm{H}$ NMR (400 MHz, $\left.\mathrm{CDCl}_{3}\right) \delta$ $8.54(\mathrm{~d}, J=4.8 \mathrm{~Hz}, 1 \mathrm{H}), 8.03(\mathrm{~d}, J=7.6 \mathrm{~Hz}, 2 \mathrm{H}), 7.60(\mathrm{td}, J=8.0,1.6 \mathrm{~Hz}, 1 \mathrm{H}), 7.49$ $(\mathrm{t}, J=7.2 \mathrm{~Hz}, 1 \mathrm{H}), 7.39$ (t, $J=7.6 \mathrm{~Hz}, 2 \mathrm{H}), 7.26(\mathrm{~d}, J=7.6 \mathrm{~Hz}, 1 \mathrm{H}), 7.12(\mathrm{dd}, J=7.2$, $5.2 \mathrm{~Hz}, 1 \mathrm{H}), 5.84-5.72(\mathrm{~m}, 1 \mathrm{H}), 5.69-5.54(\mathrm{~m}, 1 \mathrm{H}), 4.91(\mathrm{t}, J=7.2 \mathrm{~Hz}, 1 \mathrm{H}), 4.49$ (d, $J$ $=6.4 \mathrm{~Hz}, 2 \mathrm{H}), 3.74(\mathrm{~s}, 3 \mathrm{H}), 3.01(\mathrm{dt}, J=14.4,7.2 \mathrm{~Hz}, 1 \mathrm{H}), 2.70(\mathrm{dt}, J=14.0,7.2,6.8$ $\mathrm{Hz}, 1 \mathrm{H})$. 


\section{X-Ray crystal structure of $3 \mathbf{r}$}

\section{CCDC 1890277}

The crystal was obtained by slow evaporation of solution $(\mathrm{DCM} / \mathrm{PE}=10 / 1)$ of $\mathbf{3 r}$

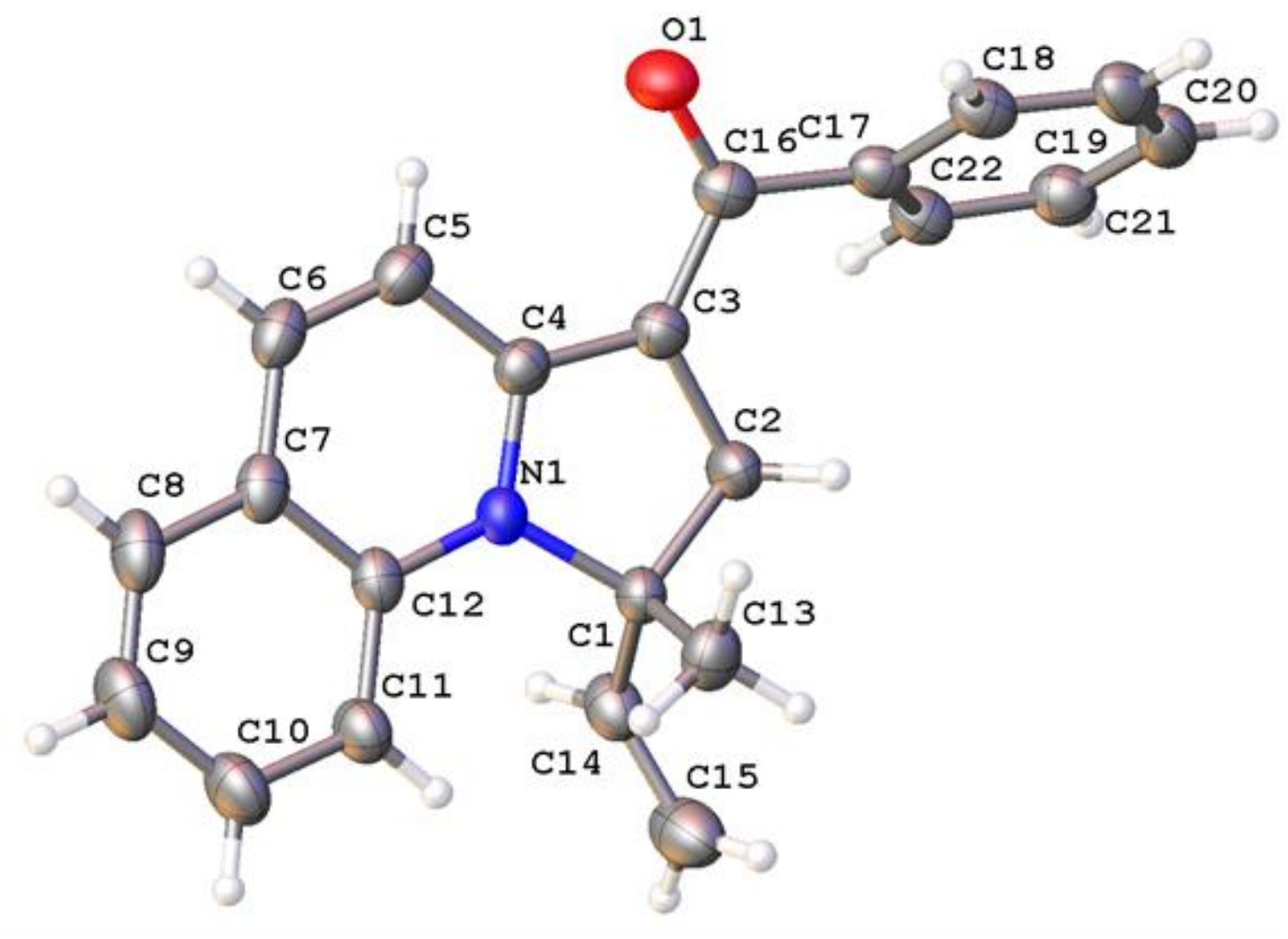

Table S4. Crystal data and structure refinement for mj118654_0m.

Identification code

Empirical formula

Formula weight

Temperature

Wavelength

Crystal system

Space group

Unit cell dimensions

Volume

$\mathrm{Z}$

Density (calculated) mj118654_0m

C22 H19 N O

313.38

$297.89 \mathrm{~K}$

$1.34139 \AA$

Monoclinic

C $12 / \mathrm{c} 1$

$\mathrm{a}=23.4482(7) \AA$

$\alpha=90^{\circ}$.

$\mathrm{b}=10.6406(3) \AA$

$\beta=129.2630(10)^{\circ}$.

$\mathrm{c}=17.9022(5) \AA$

$\gamma=90^{\circ}$.
8

$1.204 \mathrm{Mg} / \mathrm{m}^{3}$ 
Absorption coefficient

$\mathrm{F}(000)$

Crystal size

Theta range for data collection

Index ranges

Reflections collected

Independent reflections

Completeness to theta $=53.594^{\circ}$

Absorption correction

Max. and min. transmission

Refinement method

Data / restraints / parameters

Goodness-of-fit on $\mathrm{F}^{2}$

Final $\mathrm{R}$ indices [I $>2 \operatorname{sigma}(\mathrm{I})]$

$\mathrm{R}$ indices (all data)

Extinction coefficient

Largest diff. peak and hole
$0.367 \mathrm{~mm}^{-1}$

1328

$0.2 \times 0.18 \times 0.15 \mathrm{~mm}^{3}$

4.190 to $54.927^{\circ}$.

$-28<=\mathrm{h}<=28,-11<=\mathrm{k}<=12,-21<=\mathrm{l}<=21$

12776

$3271[\mathrm{R}(\mathrm{int})=0.0540]$

$99.5 \%$

Semi-empirical from equivalents

0.7508 and 0.5759

Full-matrix least-squares on $\mathrm{F}^{2}$

$3271 / 0 / 218$

1.069

$\mathrm{R} 1=0.0570, \mathrm{wR} 2=0.1588$

$\mathrm{R} 1=0.0713, \mathrm{wR} 2=0.1716$

$\mathrm{n} / \mathrm{a}$

0.475 and -0.238 e. $\AA^{-3}$ 
X-Ray crystal structure of $3 \mathrm{~s} \cdot \mathrm{HCl}$

\section{CCDC 1905118}

The crystal was obtained by slow evaporation of solution $(\mathrm{DCM} / \mathrm{n}$-pentane $=10 / 1)$ of $3 \mathbf{s} \cdot \mathrm{HCl}$

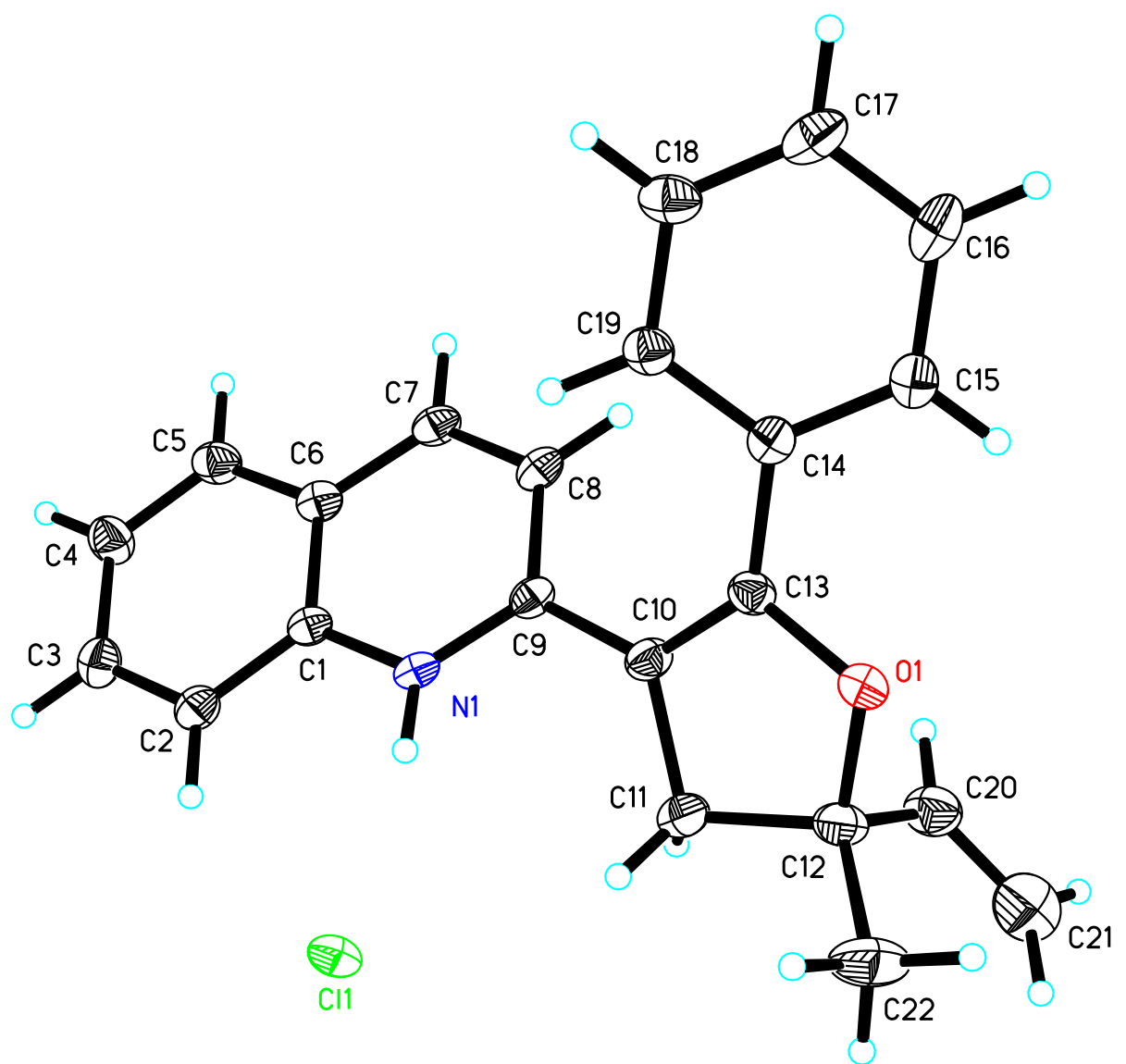

Table S5. Crystal data and structure refinement for d8v19161.

Identification code

Empirical formula

Formula weight

Temperature

Wavelength d8v19161

$\mathrm{C} 22 \mathrm{H} 20 \mathrm{Cl} \mathrm{N} \mathrm{O}$

349.84

193(2) K

$0.71073 \AA$ 
Crystal system

Space group

Unit cell dimensions

Volume

$\mathrm{Z}$

Density (calculated)

Absorption coefficient

$\mathrm{F}(000)$

Crystal size

Theta range for data collection

Index ranges

Reflections collected

Independent reflections

Completeness to theta $=25.242^{\circ}$

Absorption correction

Max. and min. transmission

Refinement method

Data / restraints / parameters

Goodness-of-fit on $\mathrm{F}^{2}$

Final $\mathrm{R}$ indices [I $>2 \operatorname{sigma}(\mathrm{I})]$

$\mathrm{R}$ indices (all data)

Absolute structure parameter

Extinction coefficient

Largest diff. peak and hole
Orthorhombic

I b a 2

$\mathrm{a}=18.4720(5) \AA \quad \alpha=90^{\circ}$.

$\mathrm{b}=29.1767(10) \AA \quad \beta=90^{\circ}$.

$\mathrm{c}=6.8903(2) \AA \quad \gamma=90^{\circ}$.

3713.54(19) $\AA^{3}$

8

$1.251 \mathrm{Mg} / \mathrm{m}^{3}$

$0.214 \mathrm{~mm}^{-1}$

1472

$0.200 \times 0.130 \times 0.090 \mathrm{~mm}^{3}$

3.451 to $25.497^{\circ}$.

$-22<=\mathrm{h}<=20,-35<=\mathrm{k}<=35,-8<=\mathrm{l}<=8$

17889

$3440[\mathrm{R}(\mathrm{int})=0.0376]$

$99.1 \%$

Semi-empirical from equivalents

0.7456 and 0.6700

Full-matrix least-squares on $\mathrm{F}^{2}$

3440 / 34 / 258

1.059

$\mathrm{R} 1=0.0390, \mathrm{wR} 2=0.1058$

$\mathrm{R} 1=0.0422, \mathrm{wR} 2=0.1089$

$-0.02(3)$

$\mathrm{n} / \mathrm{a}$

0.602 and -0.220 e. $\AA^{-3}$ 


\section{Copies of NMR spectra}

${ }^{1} \mathrm{H}$ NMR spectra of $\mathbf{1 a}$

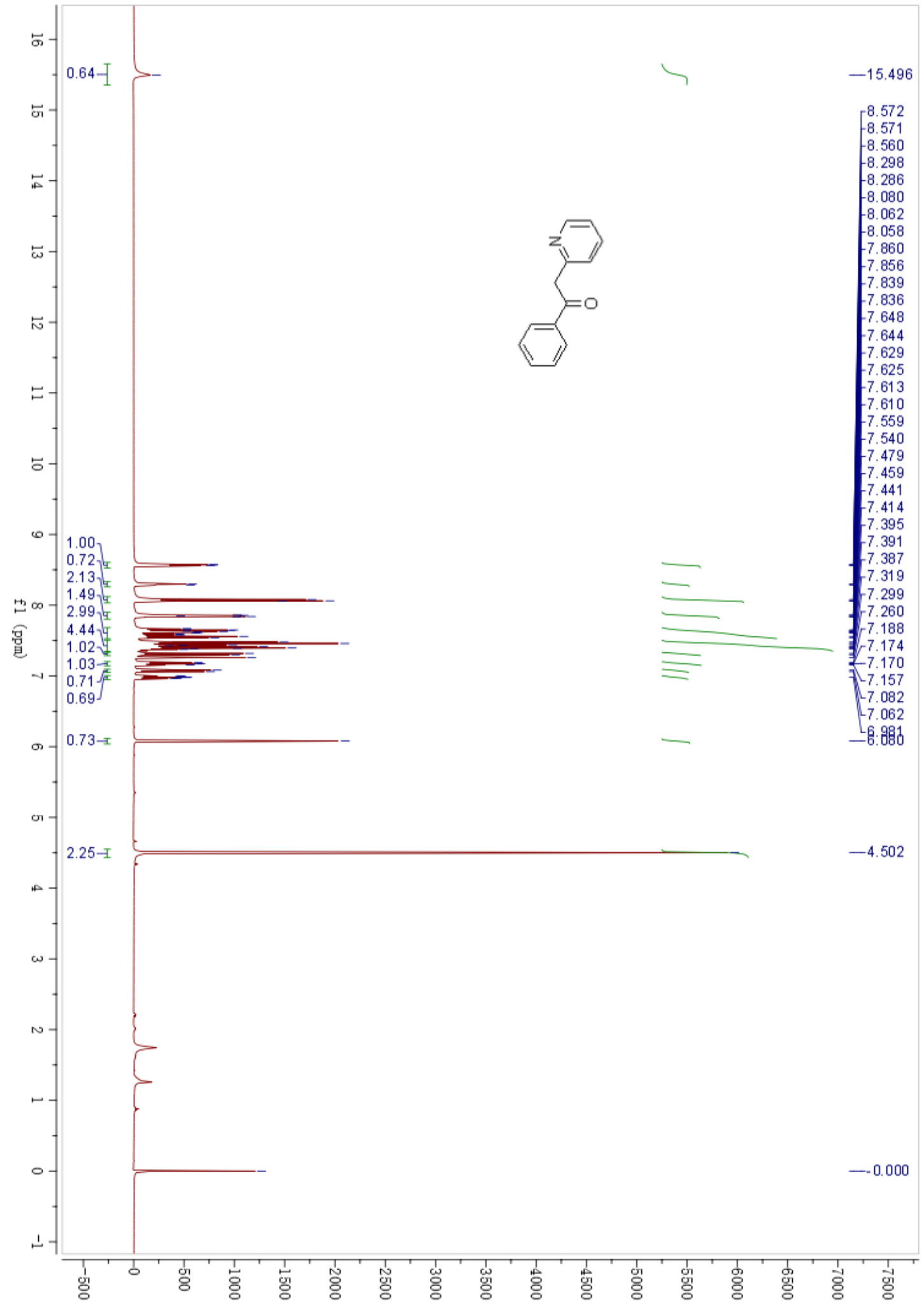


${ }^{1} \mathrm{H}$ NMR spectra of $\mathbf{1 b}$

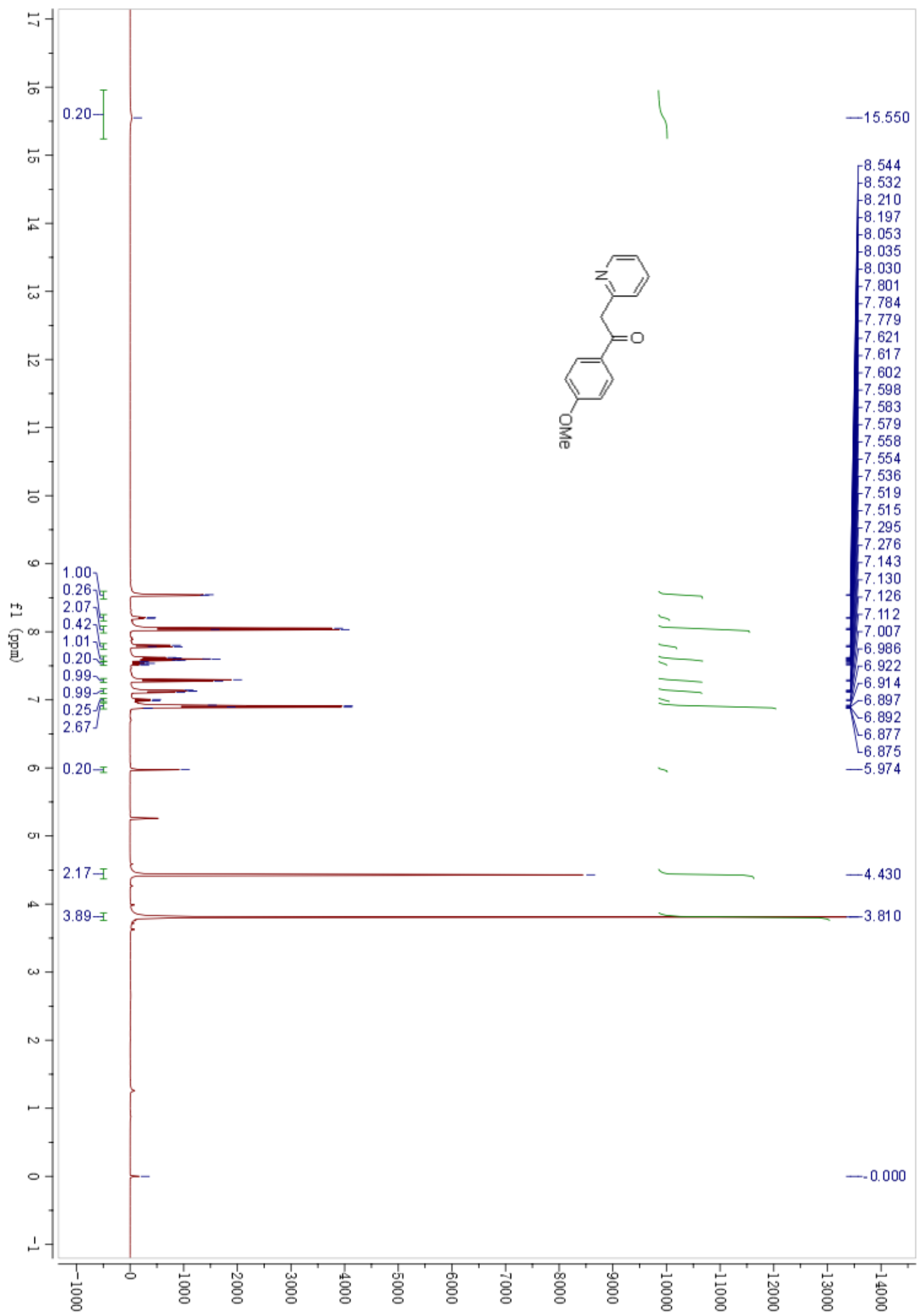


${ }^{1} \mathrm{H}$ NMR spectra of $\mathbf{1 c}$

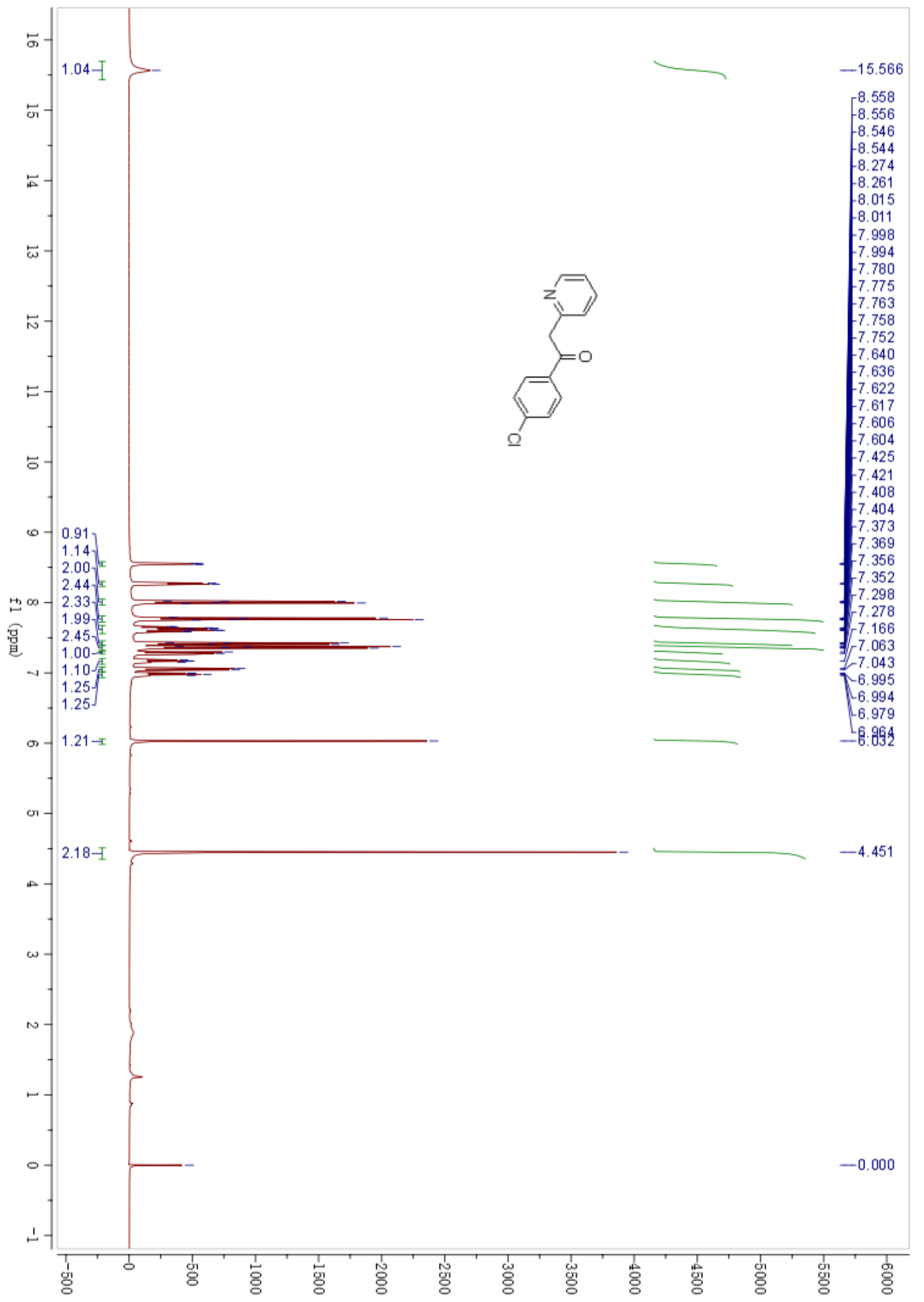


${ }^{1} \mathrm{H}$ NMR spectra of $\mathbf{1 d}$

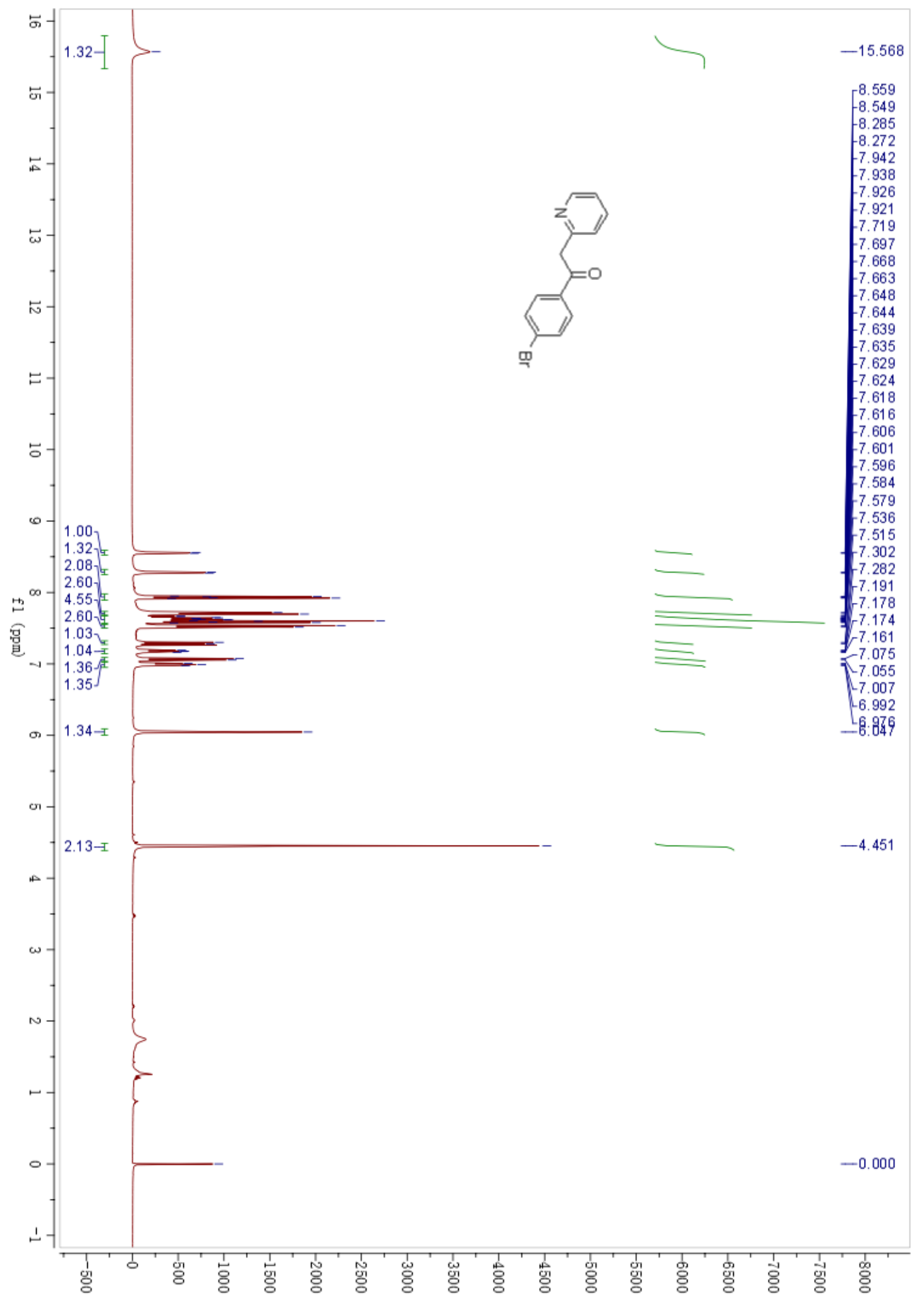


${ }^{1} \mathrm{H}$ NMR spectra of $\mathbf{1 e}$

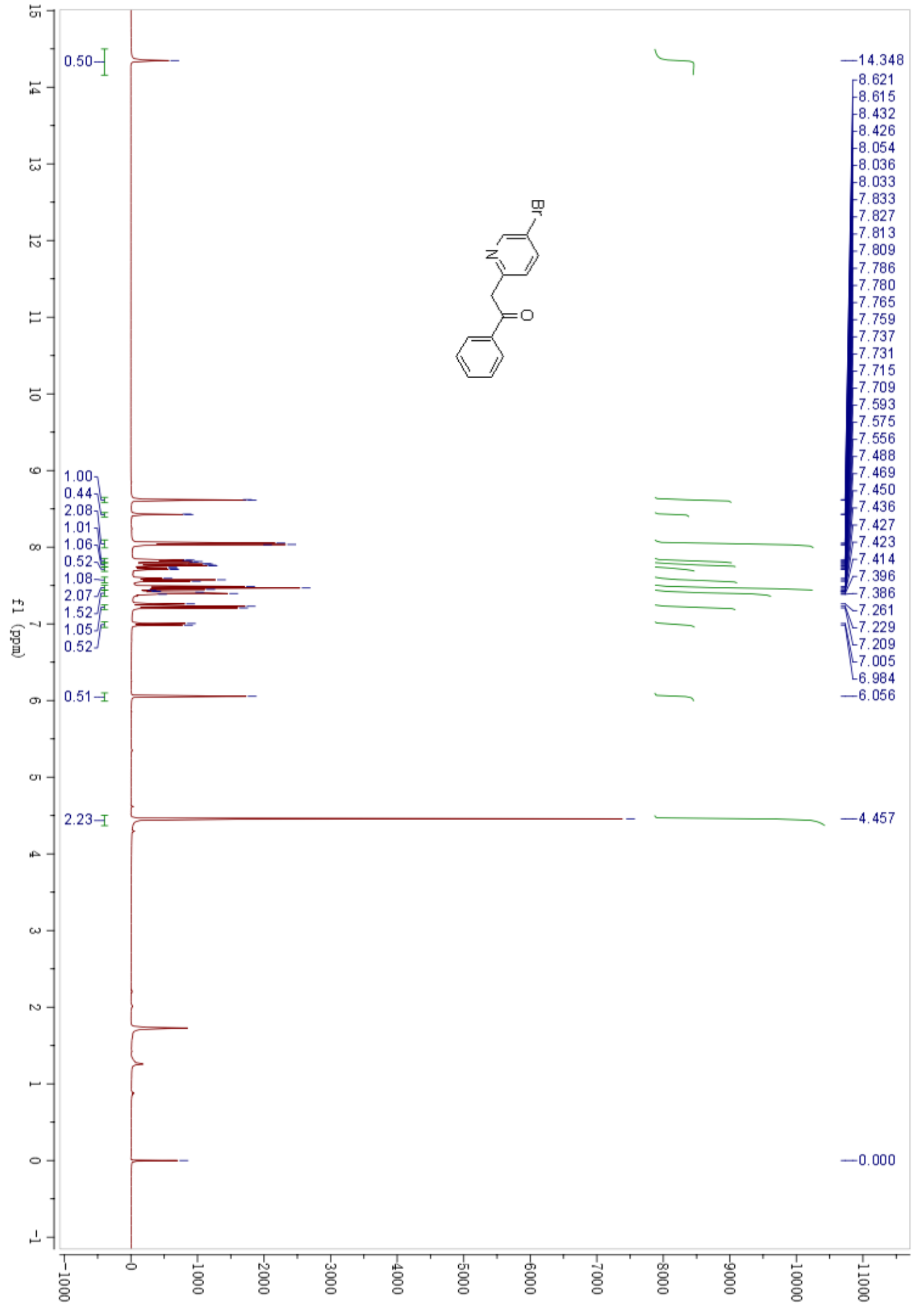


${ }^{13} \mathrm{C}$ NMR spectra of $\mathbf{1 e}$

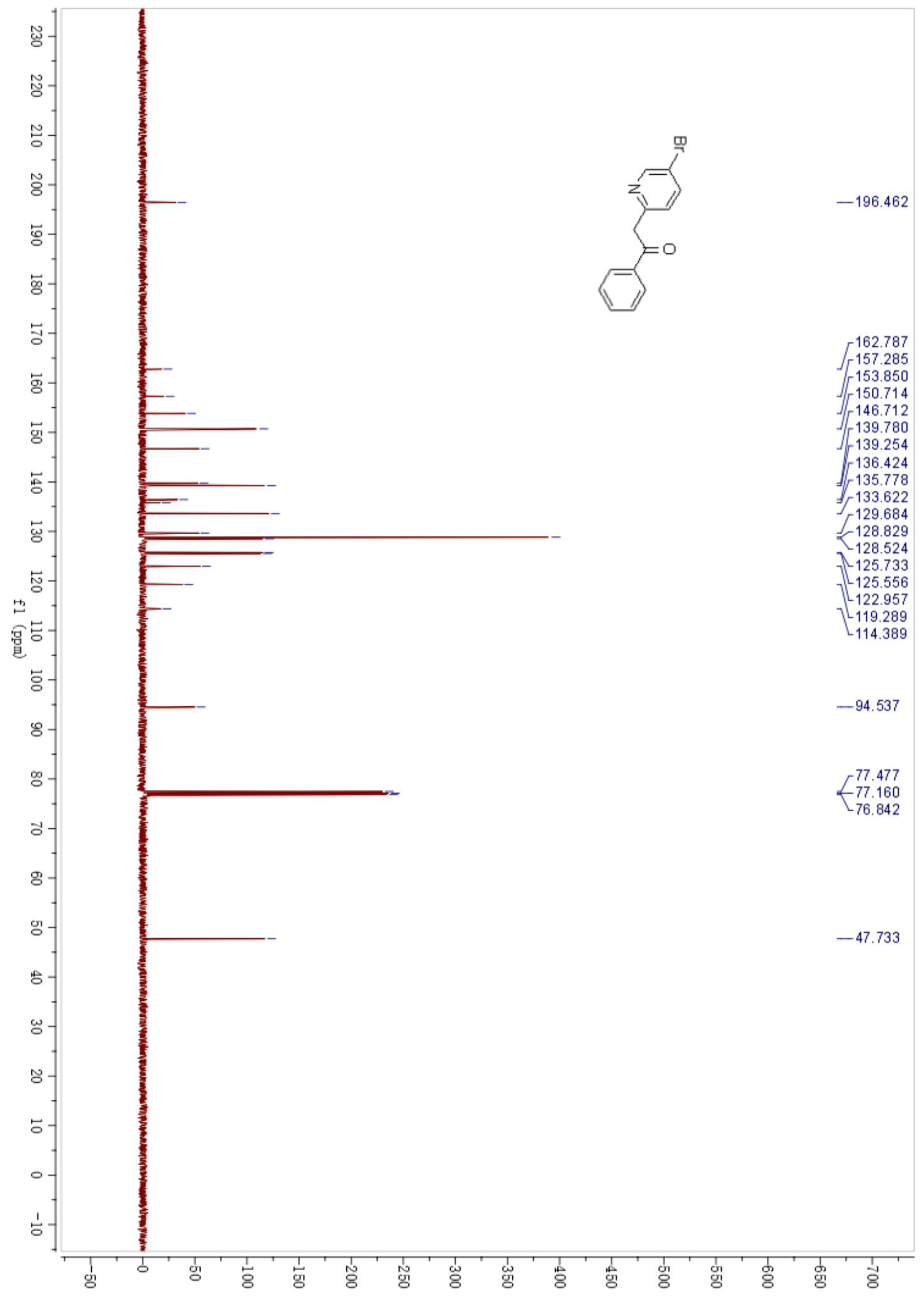


${ }^{1} \mathrm{H}$ NMR spectra of $\mathbf{1 f}$

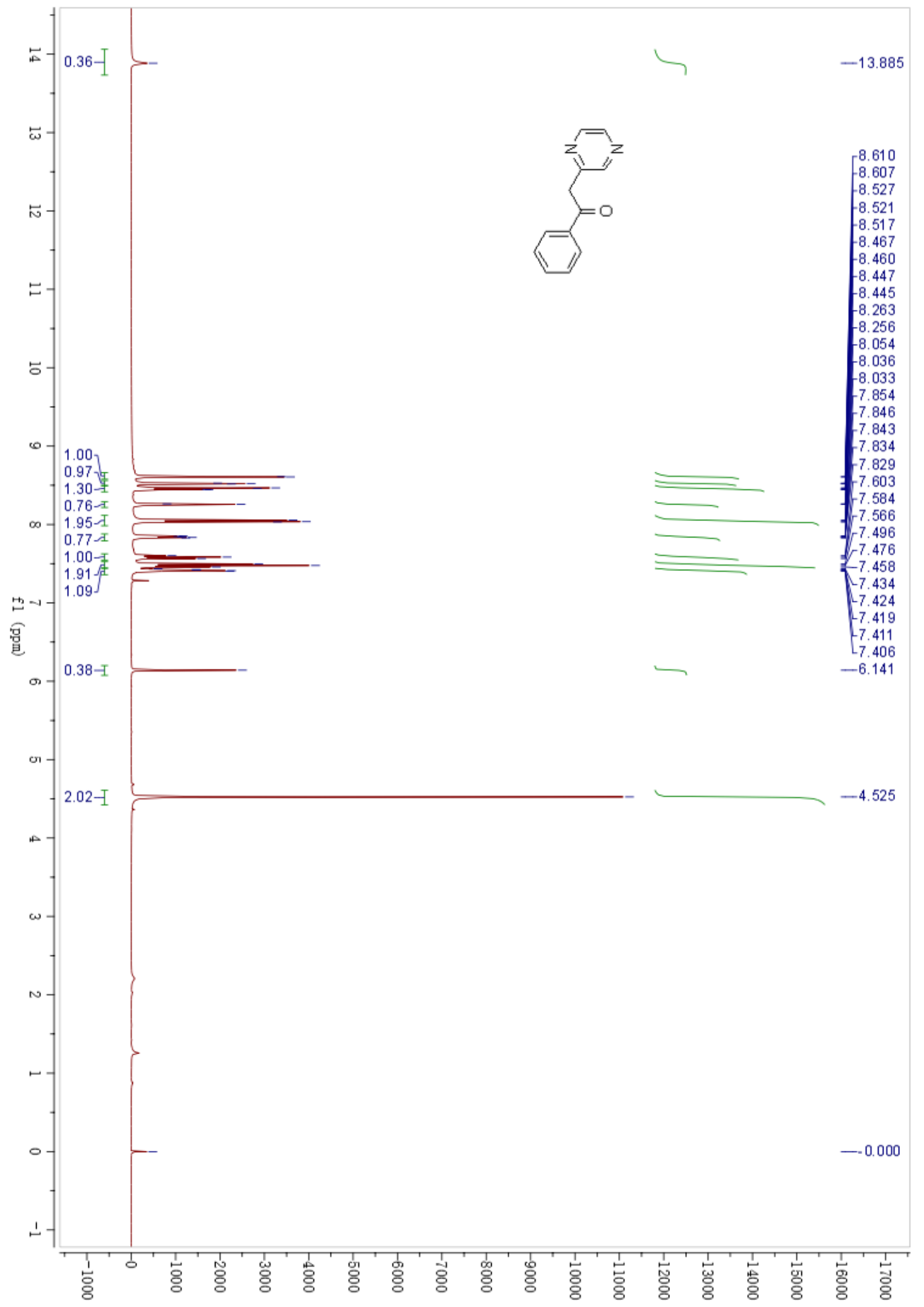


${ }^{1} \mathrm{H}$ NMR spectra of $\mathbf{1 g}$

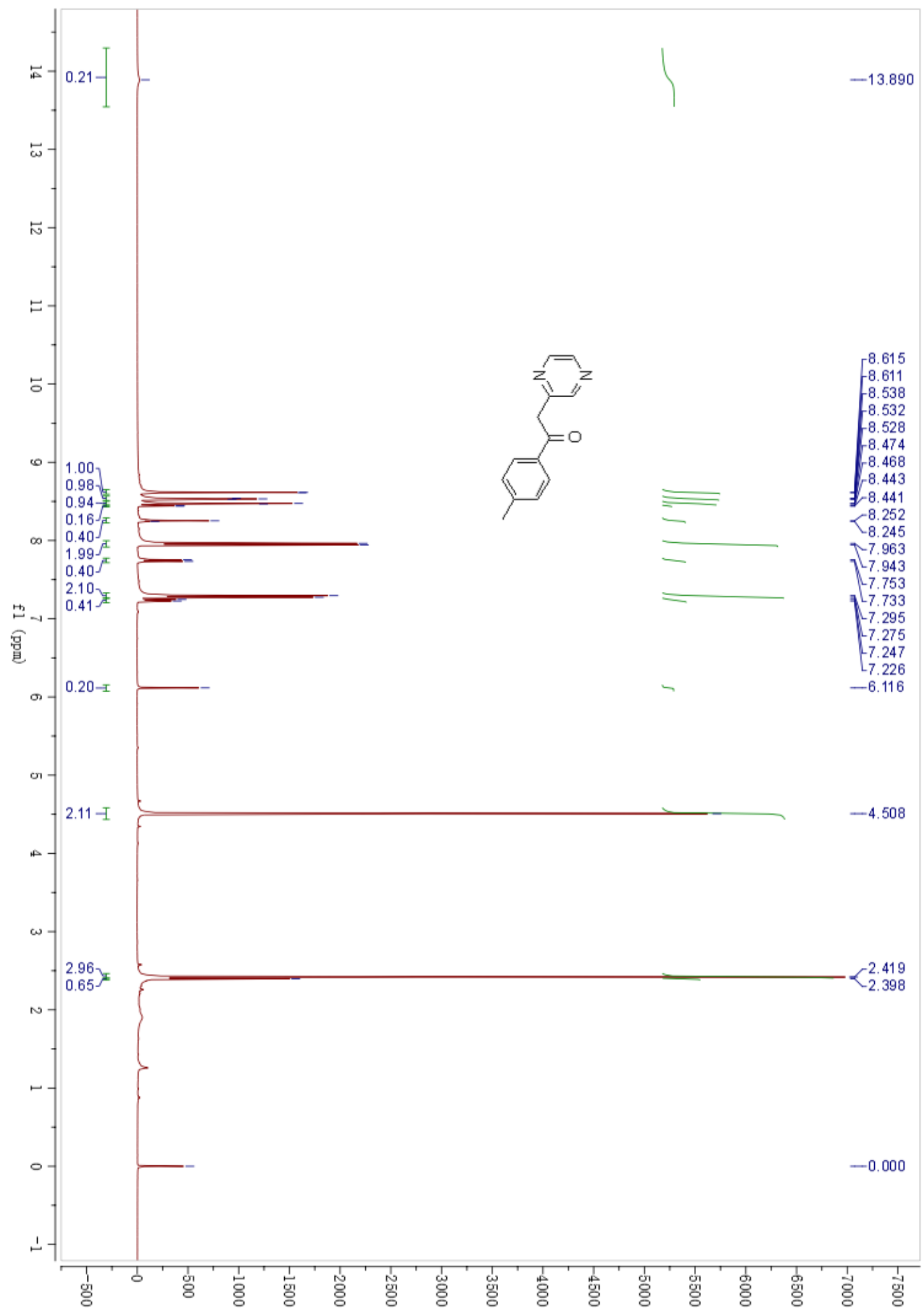


${ }^{13} \mathrm{C}$ NMR spectra of $\mathbf{1 g}$

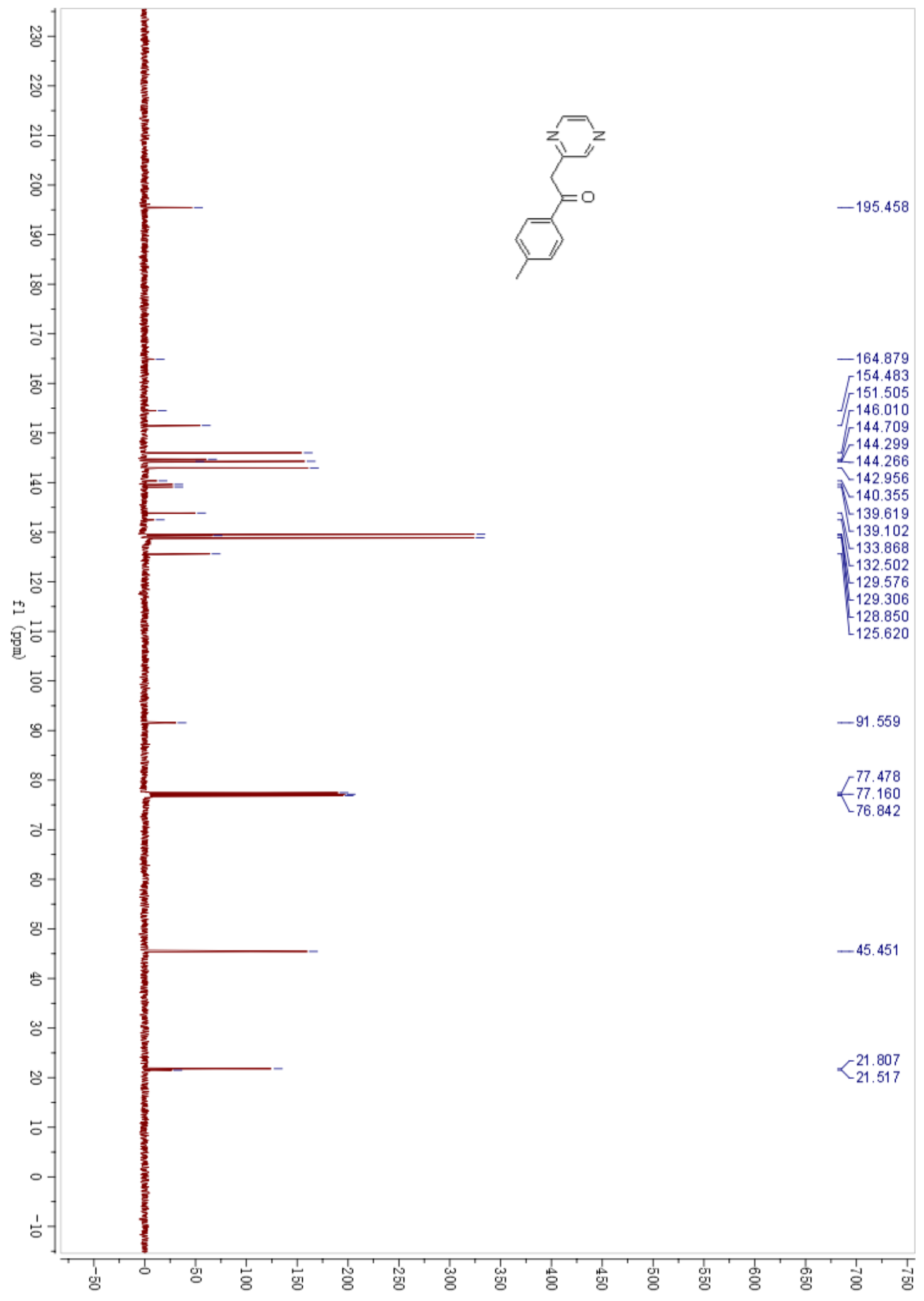


${ }^{1} \mathrm{H}$ NMR spectra of $\mathbf{1 h}$

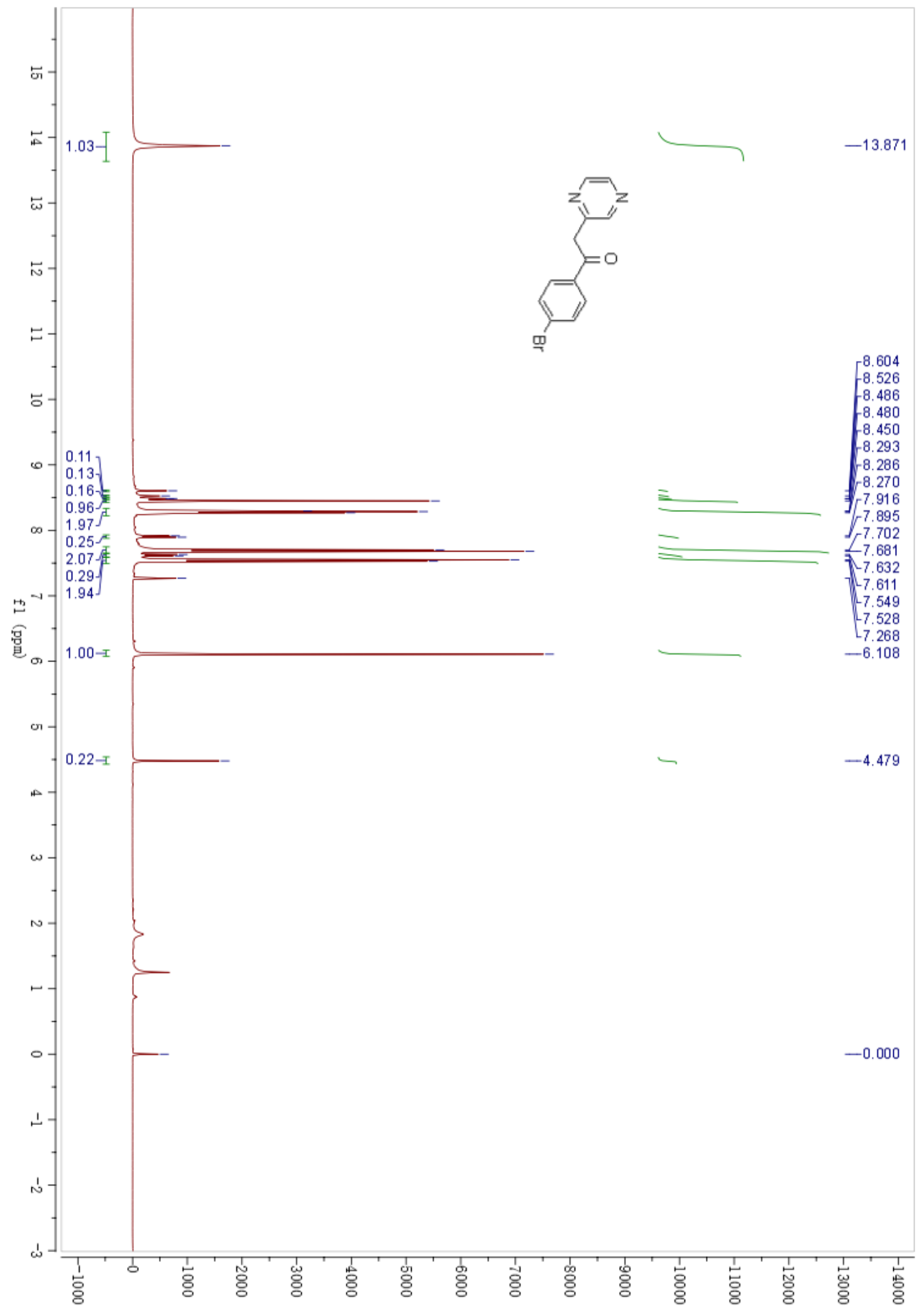


${ }^{13} \mathrm{C}$ NMR spectra of $\mathbf{1 h}$

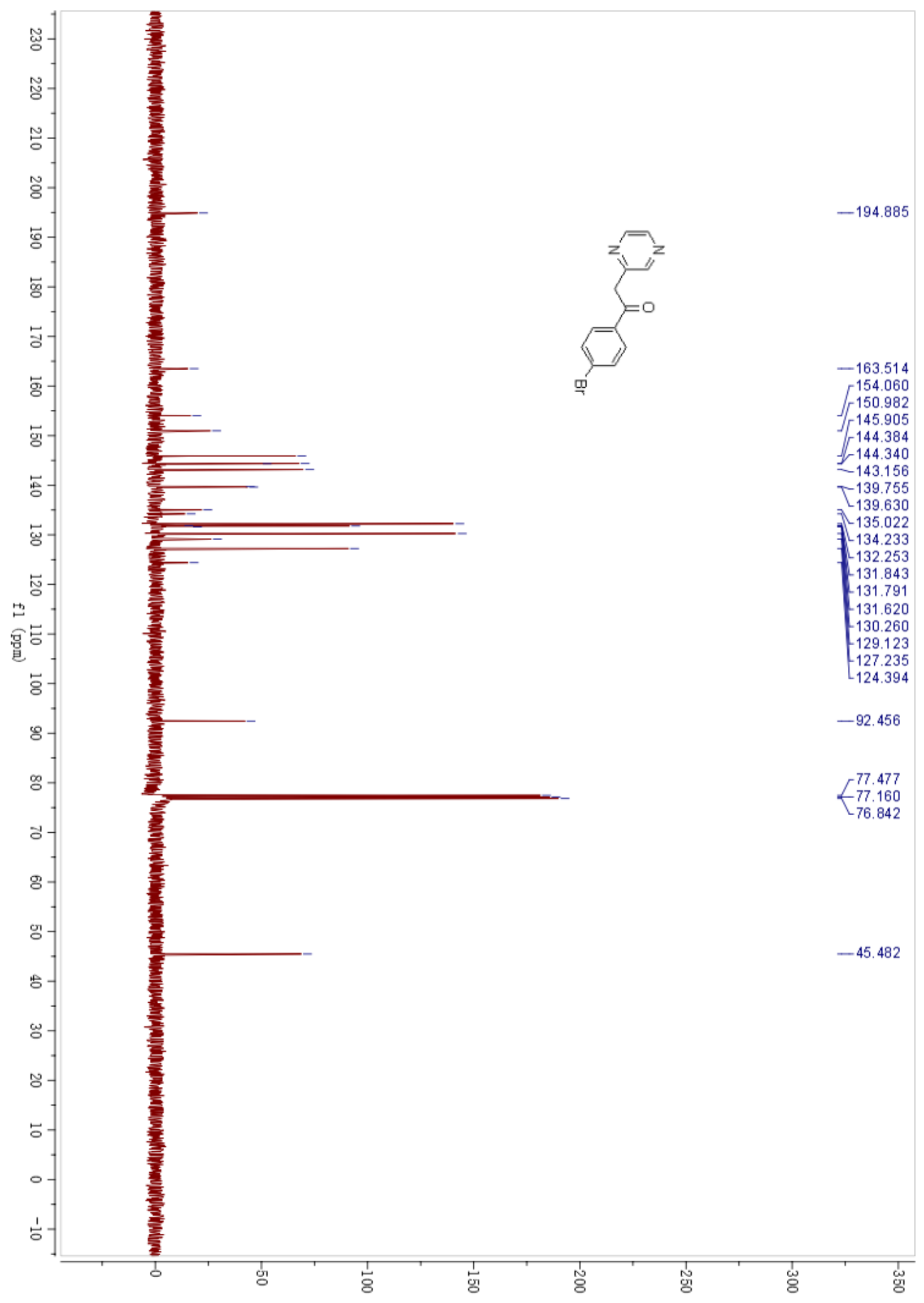


${ }^{1}$ H NMR spectra of $\mathbf{1 i}$

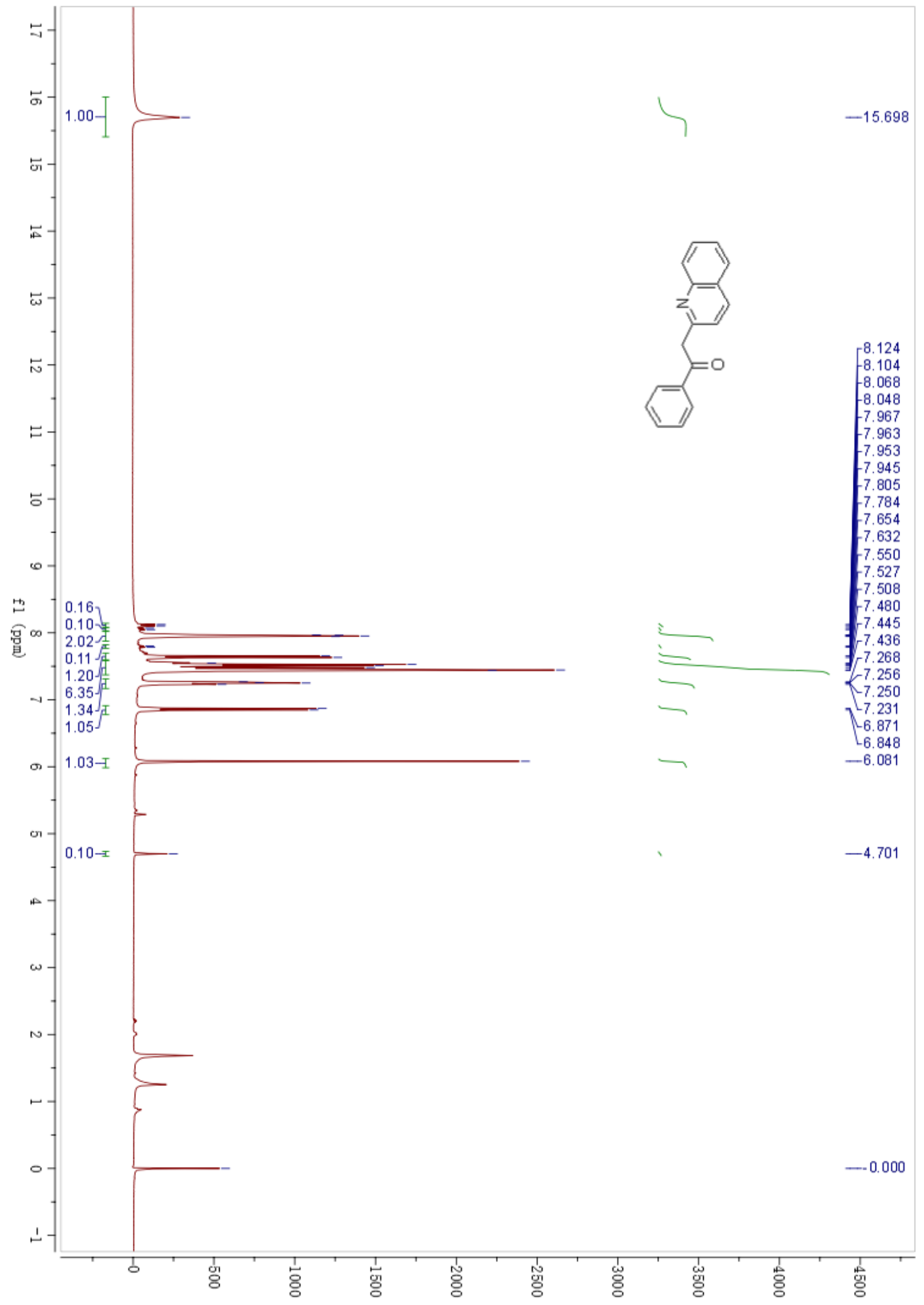


${ }^{1} \mathrm{H}$ NMR spectra of $\mathbf{1 j}$

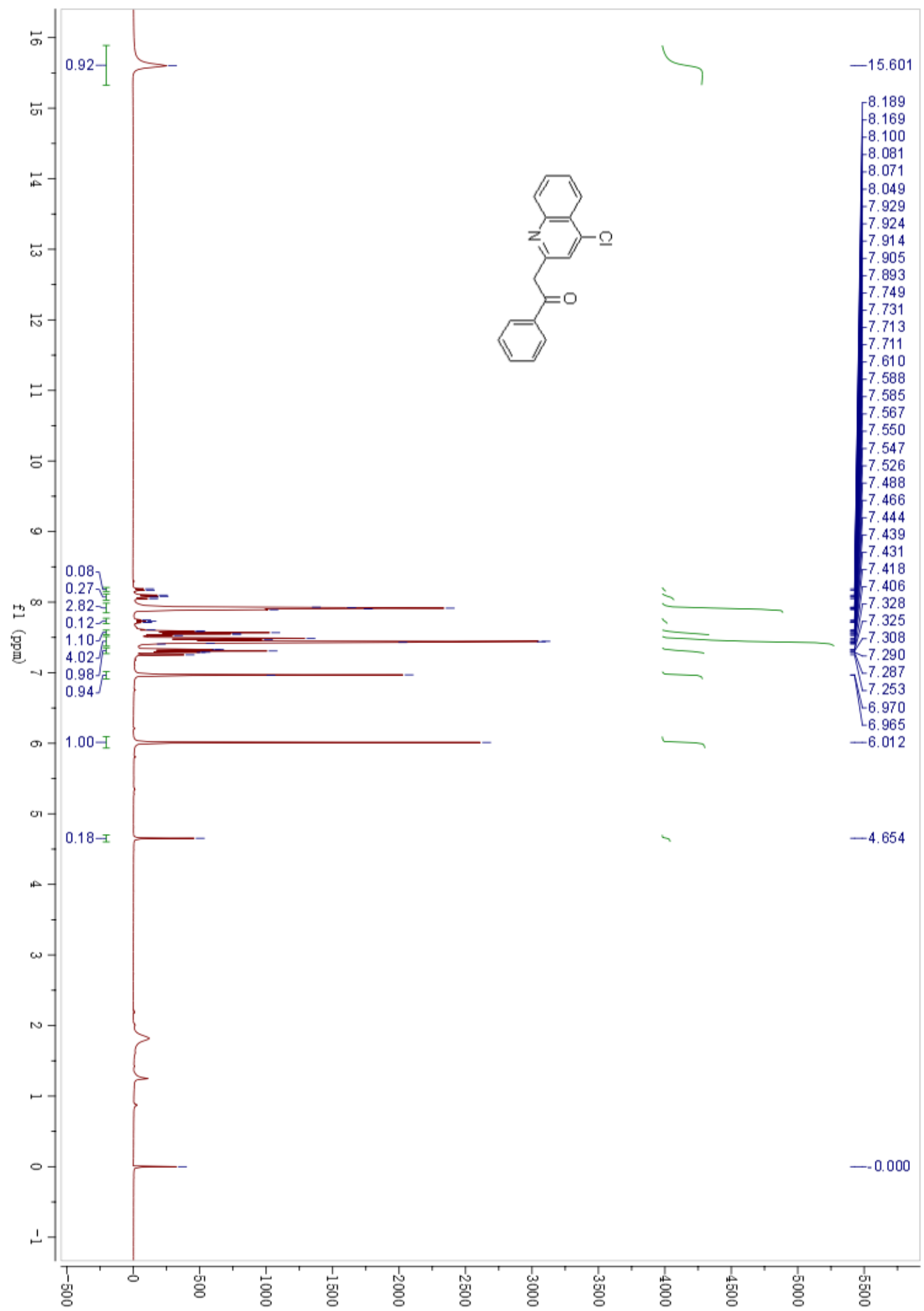


${ }^{13} \mathrm{C}$ NMR spectra of $\mathbf{1 j}$

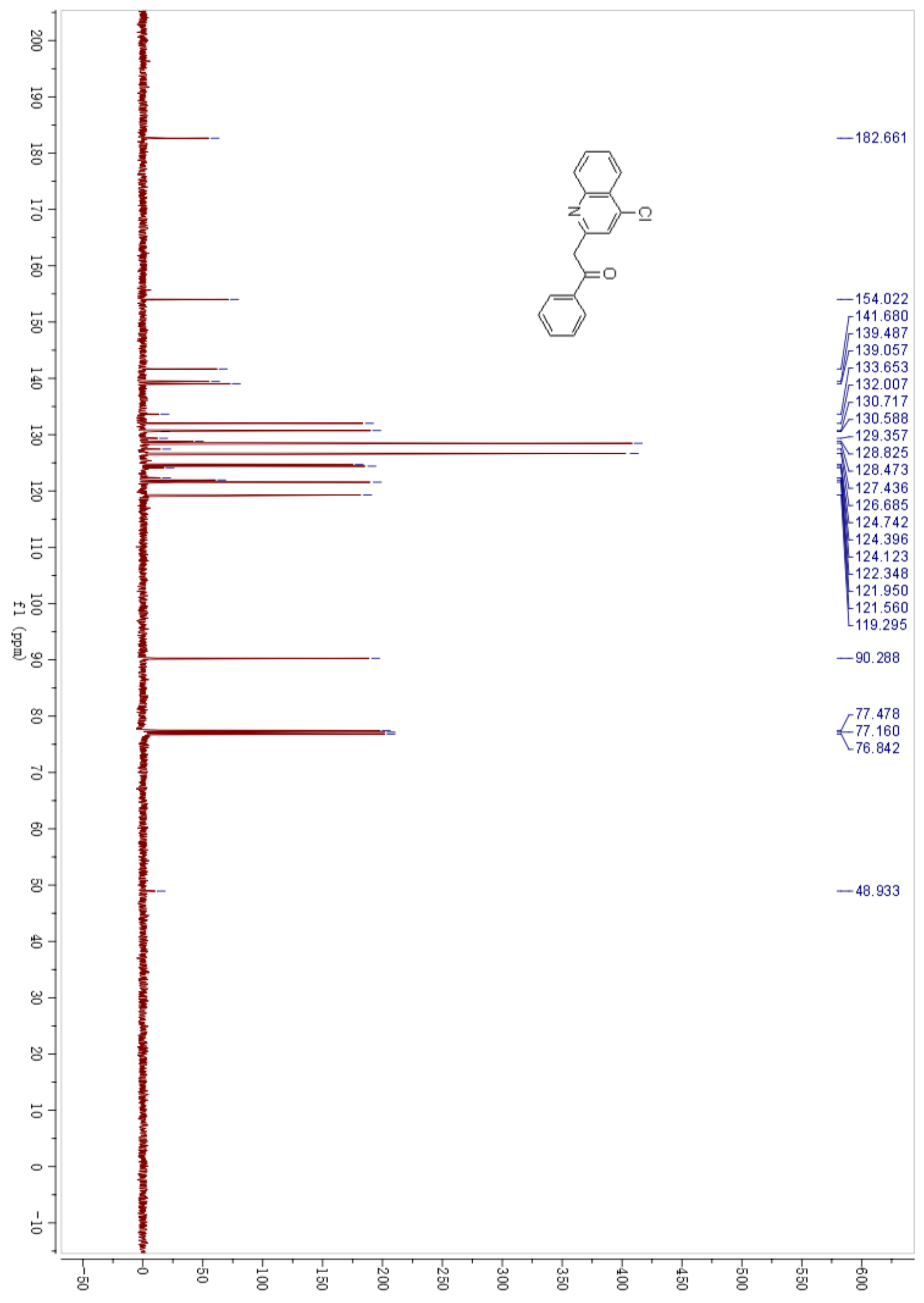


${ }^{1} \mathrm{H}$ NMR spectra of $\mathbf{1 k}$

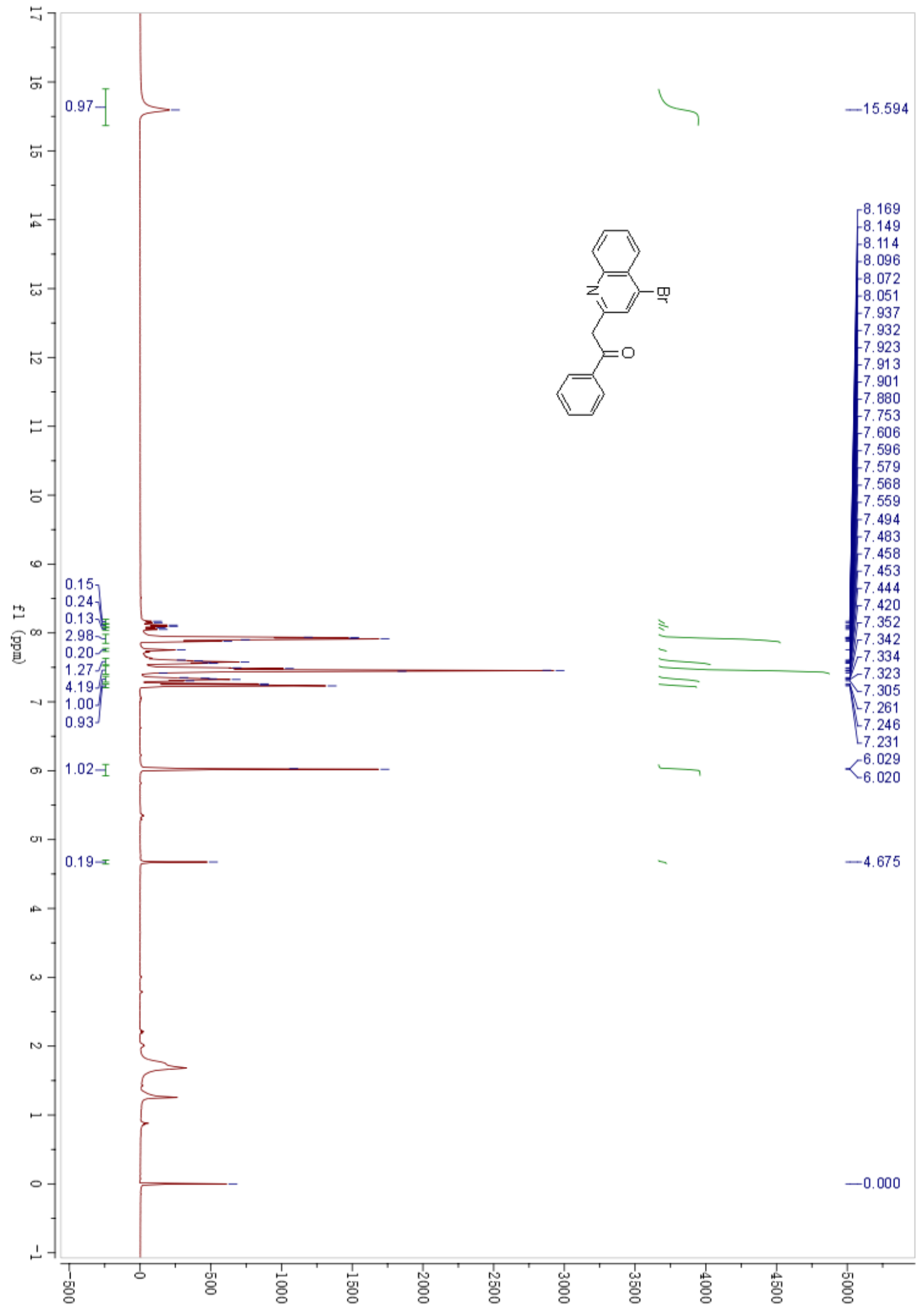


${ }^{13} \mathrm{C}$ NMR spectra of $\mathbf{1 k}$

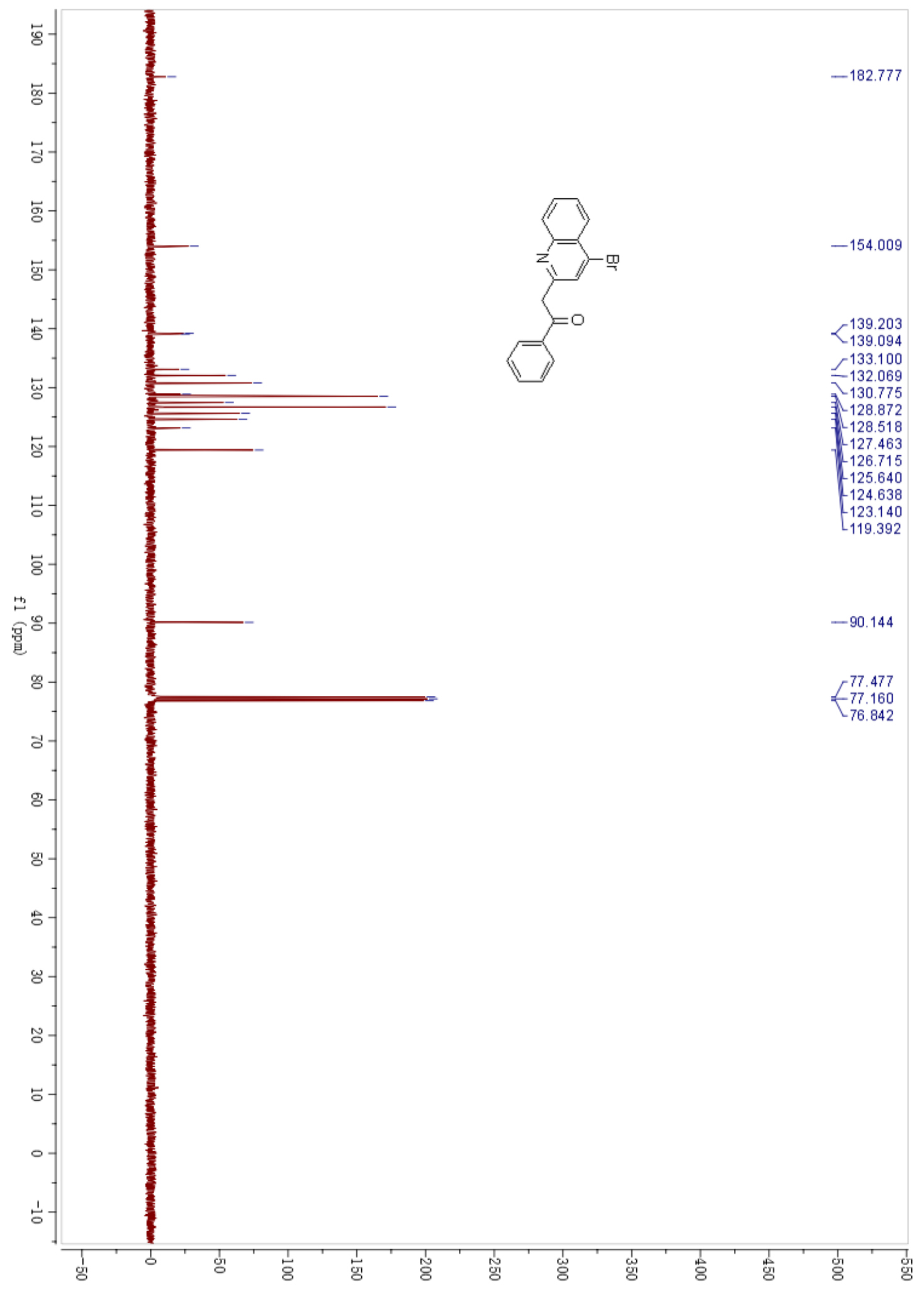


${ }^{1}$ H NMR spectra of $\mathbf{1 l}$

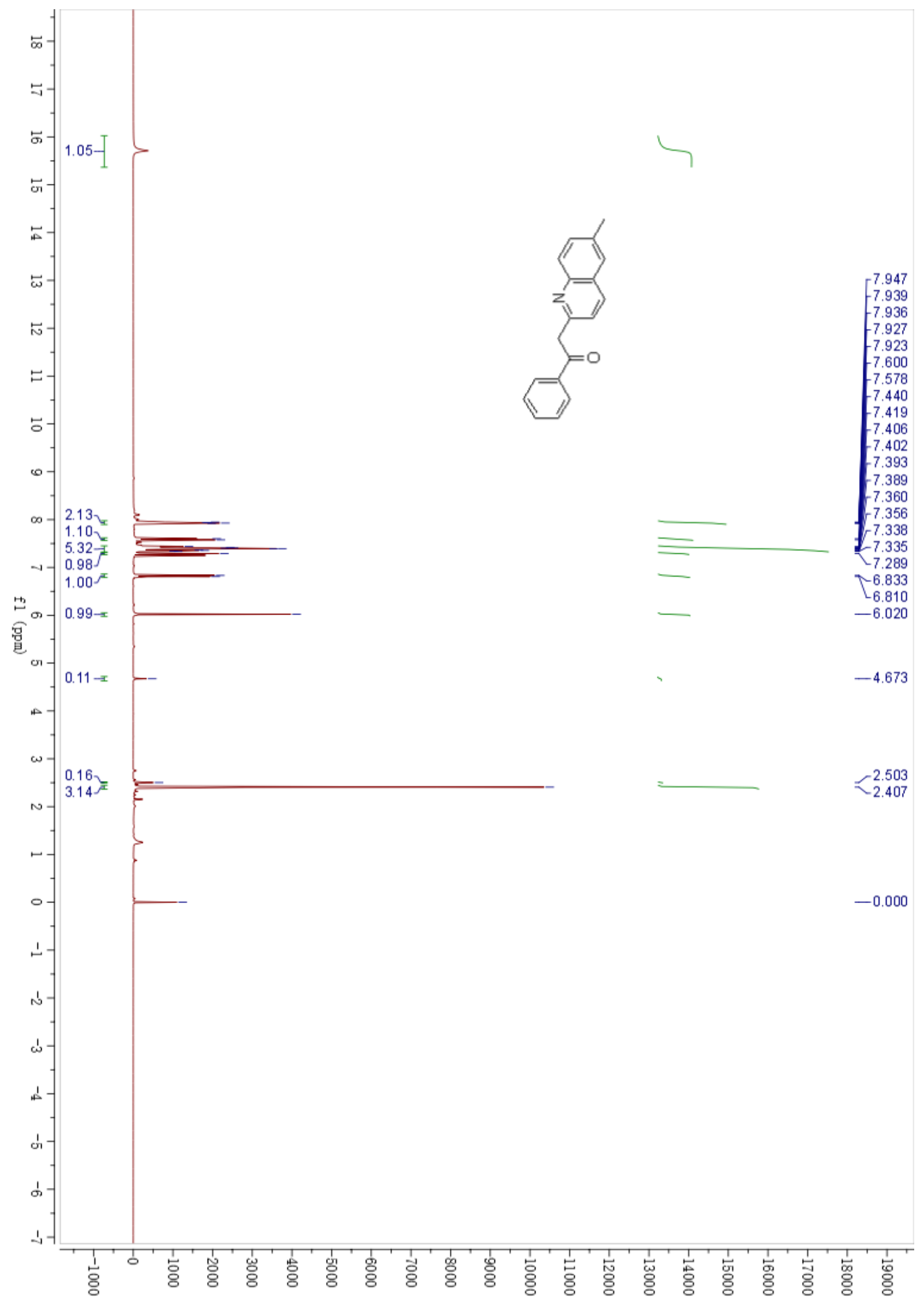


${ }^{1} \mathrm{H}$ NMR spectra of $\mathbf{1 m}$

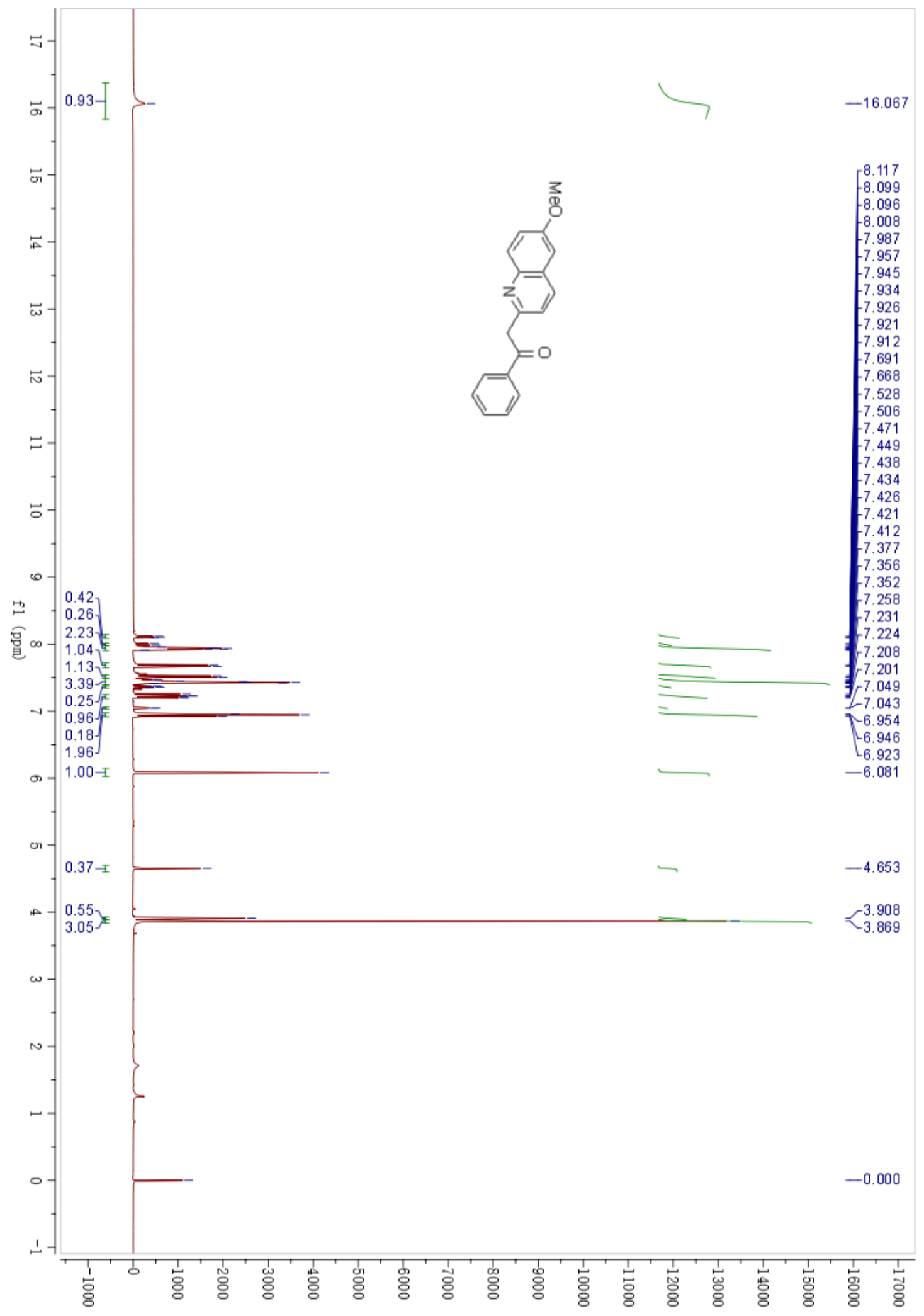


${ }^{1} \mathrm{H}$ NMR spectra of $\mathbf{1 n}$

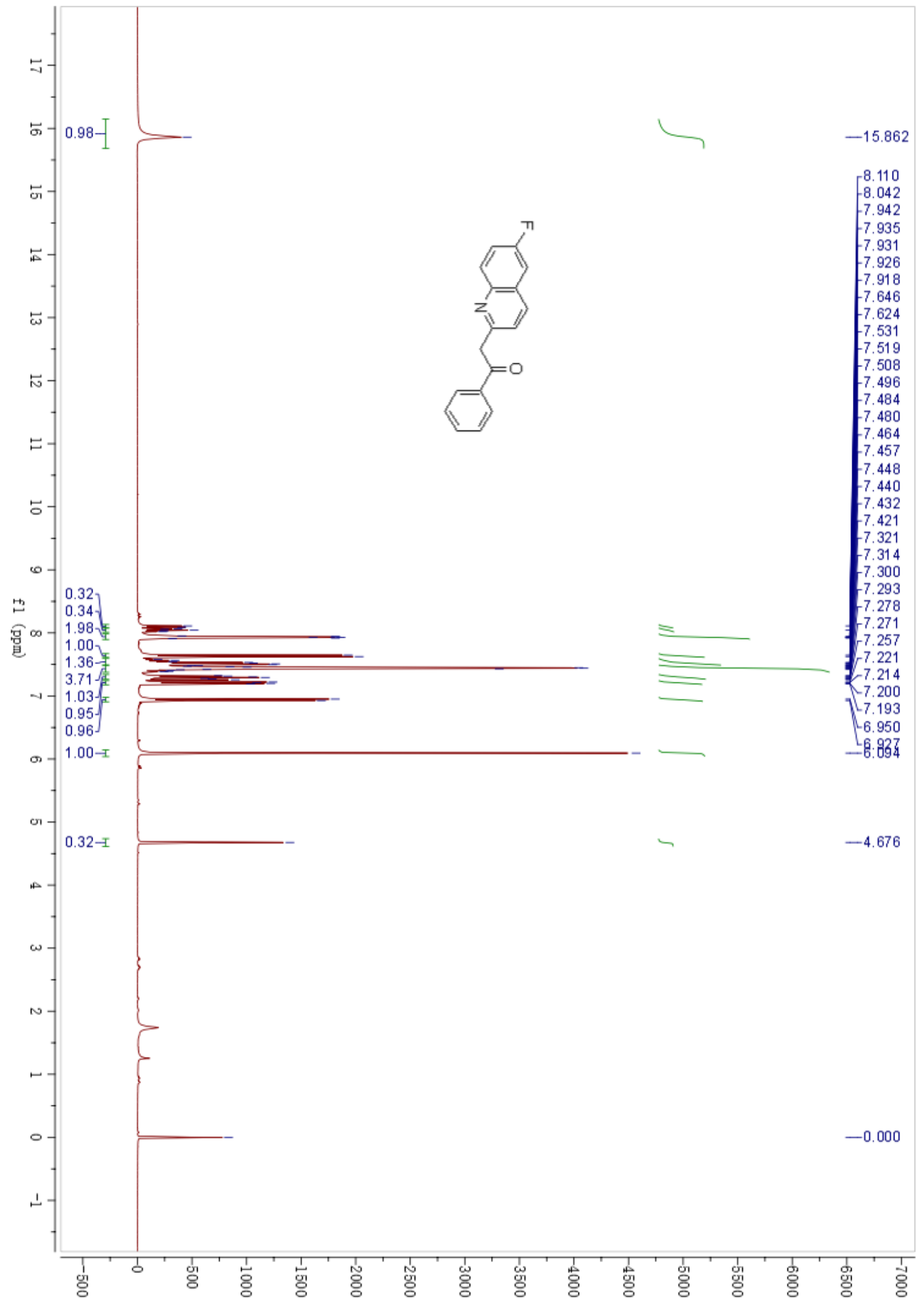


${ }^{1} \mathrm{H}$ NMR spectra of $\mathbf{1 0}$

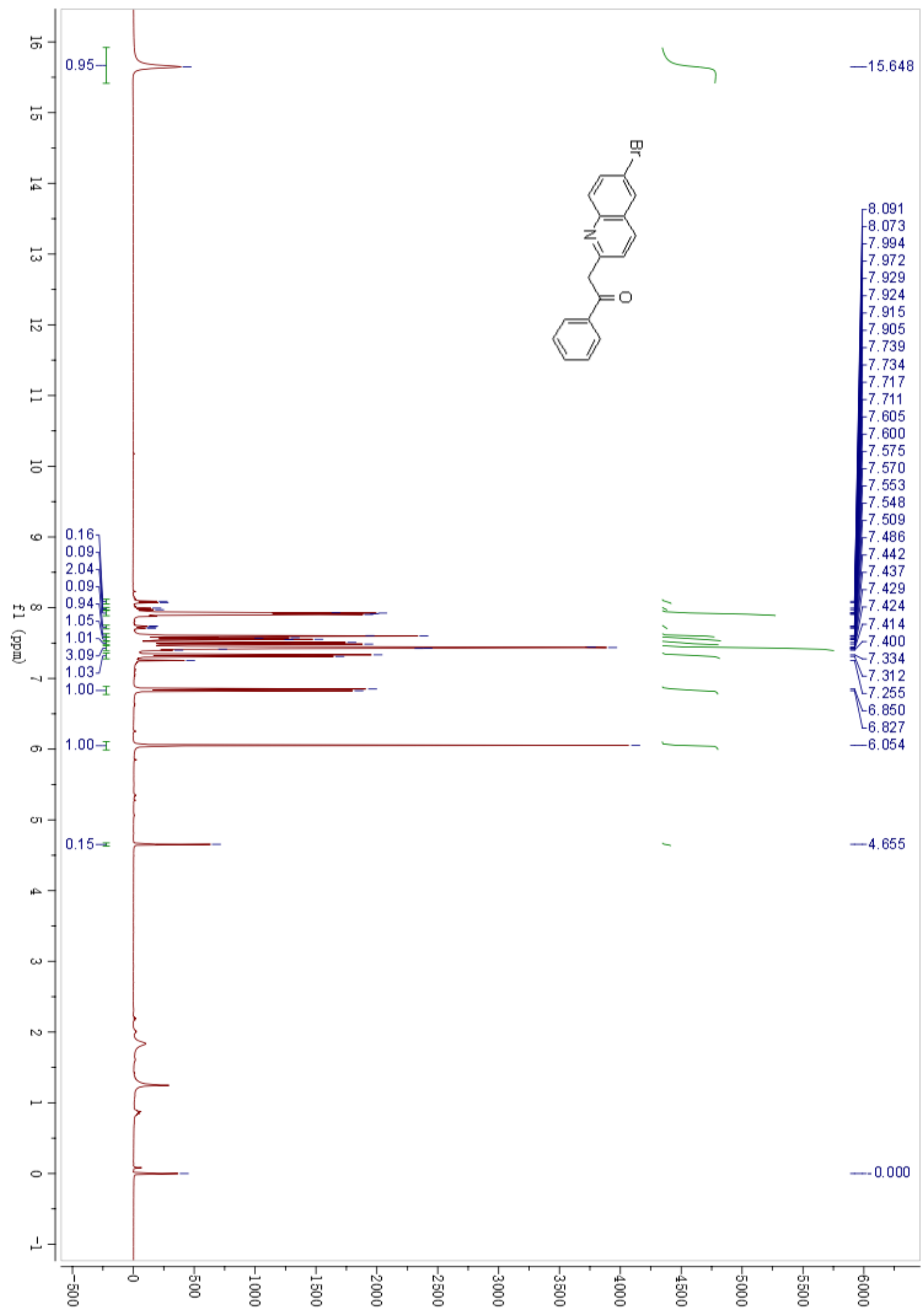


${ }^{13} \mathrm{C}$ NMR spectra of $\mathbf{1 o}$

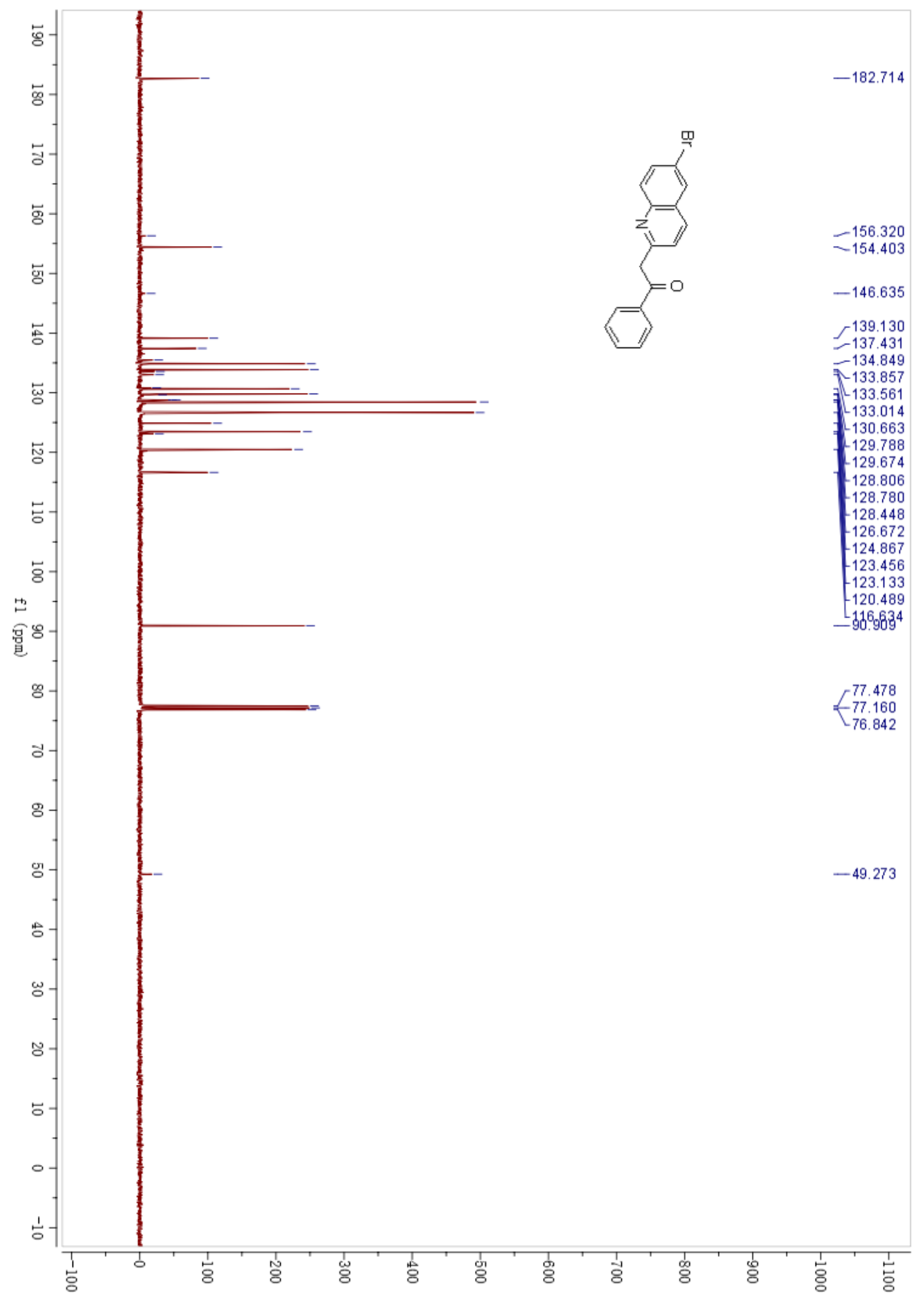


${ }^{1} \mathrm{H}$ NMR spectra of $\mathbf{1 p}$

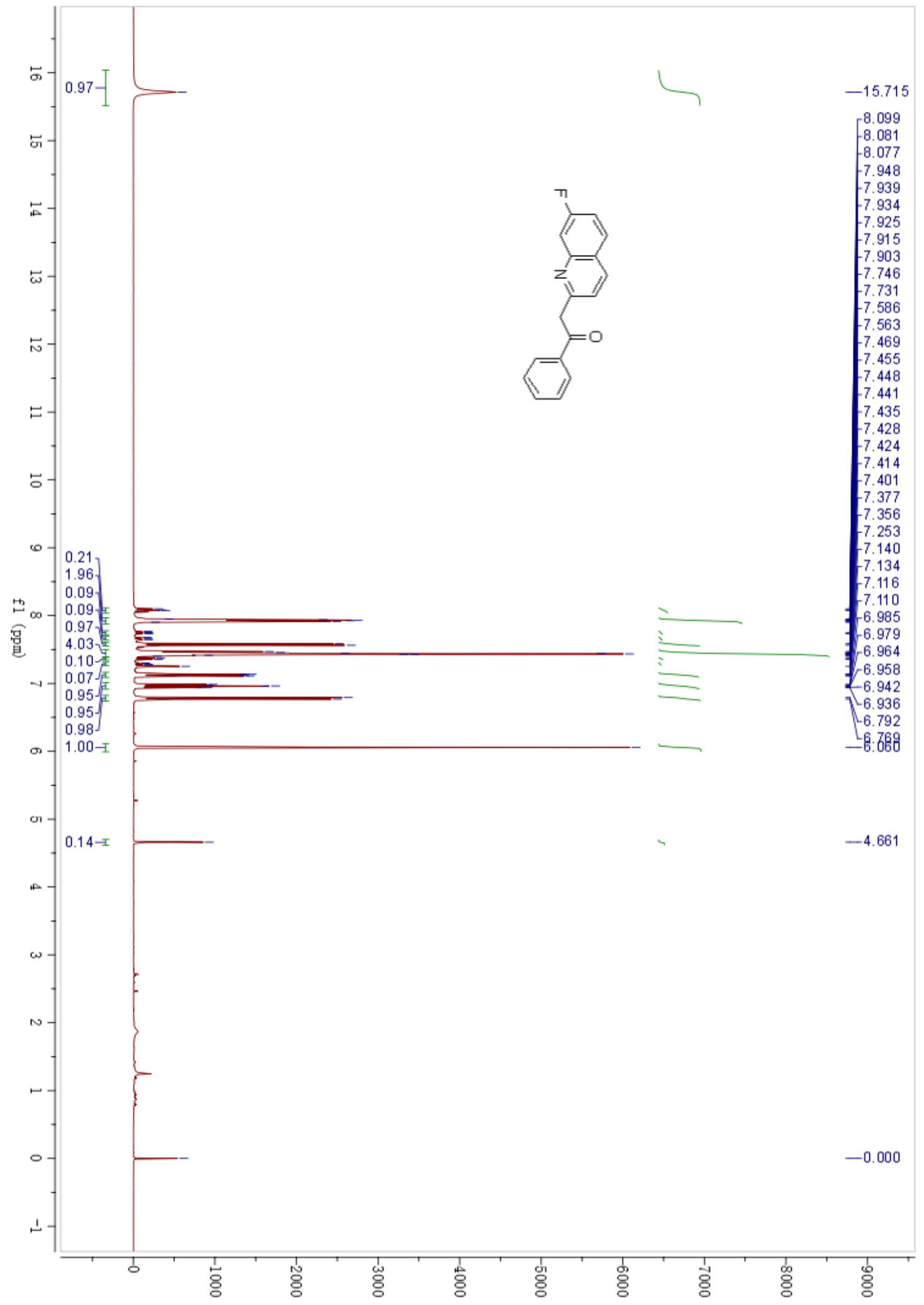


${ }^{13} \mathrm{C}$ NMR spectra of $\mathbf{1 p}$

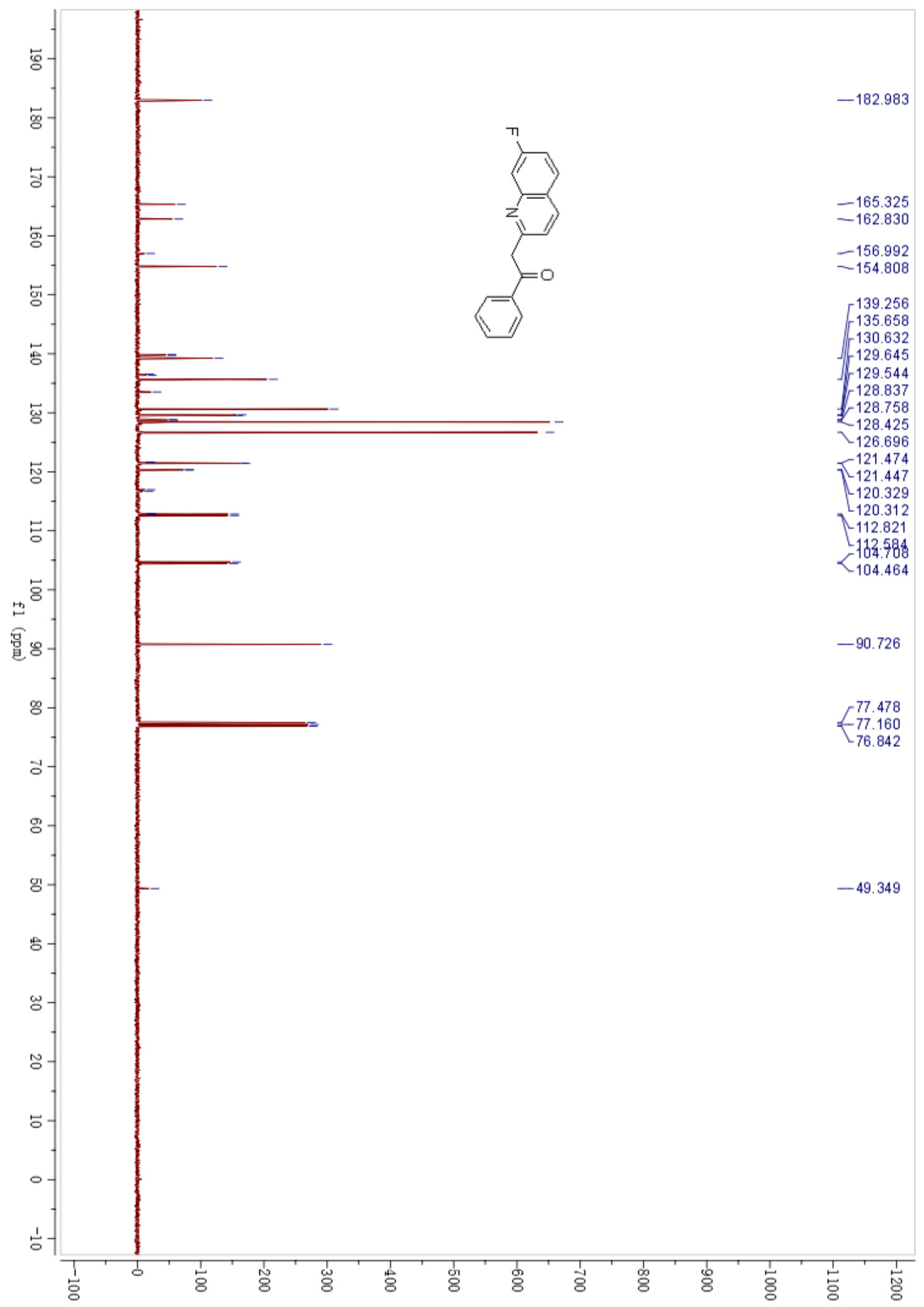


${ }^{19}$ F NMR spectra of $\mathbf{1 p}$

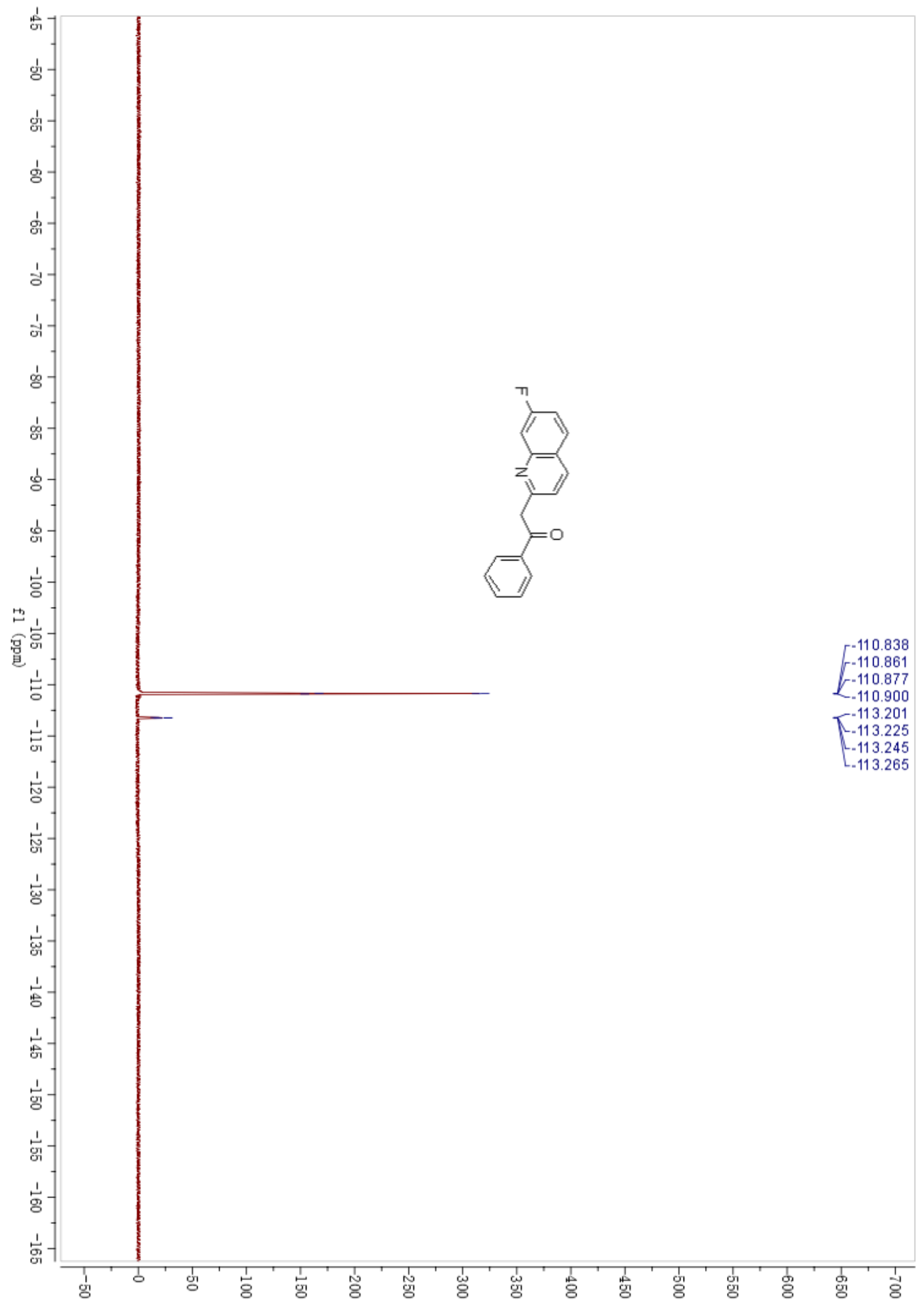


${ }^{1} \mathrm{H}$ NMR spectra of $\mathbf{1 q}$

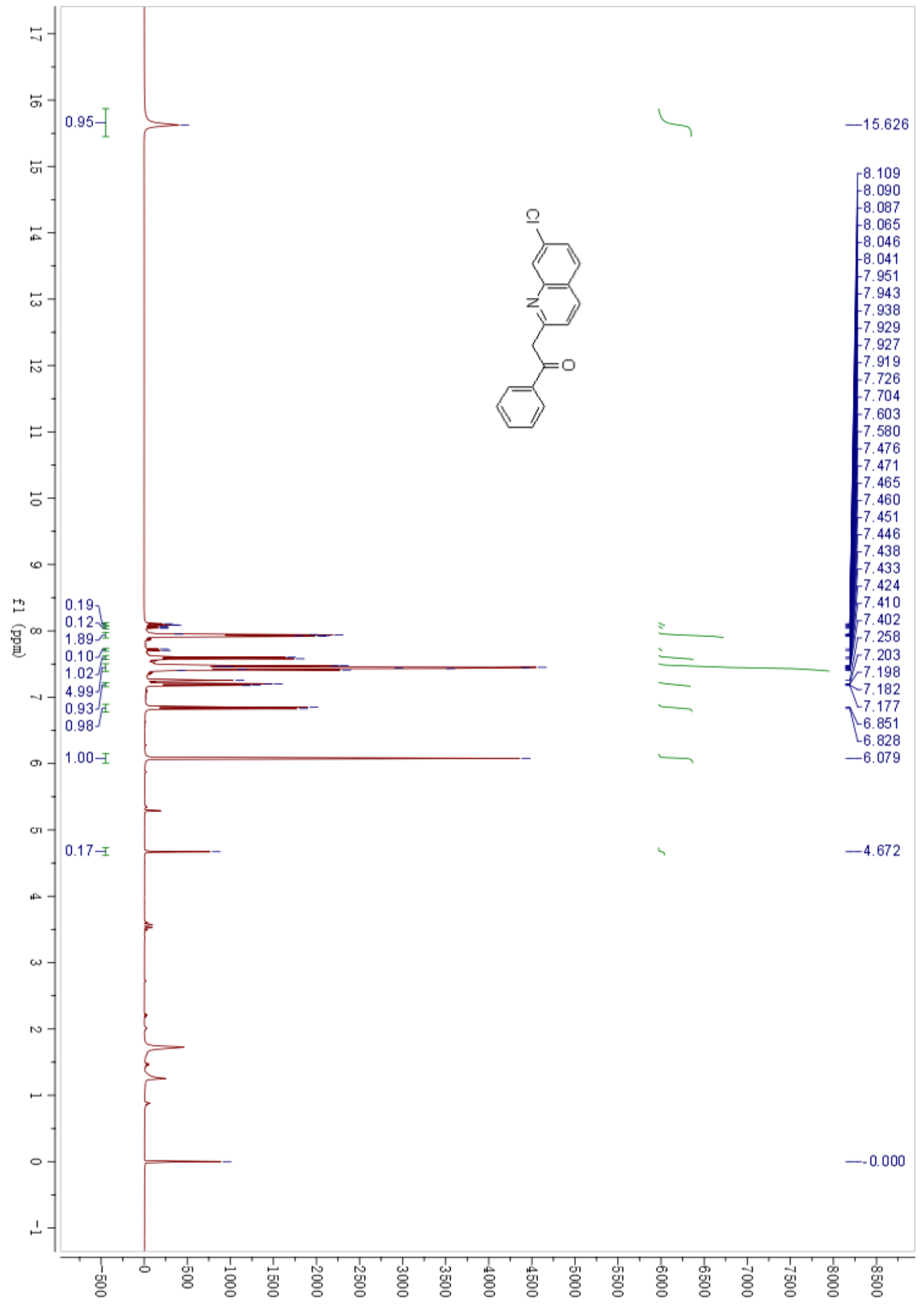


${ }^{13} \mathrm{C}$ NMR spectra of $\mathbf{1 q}$

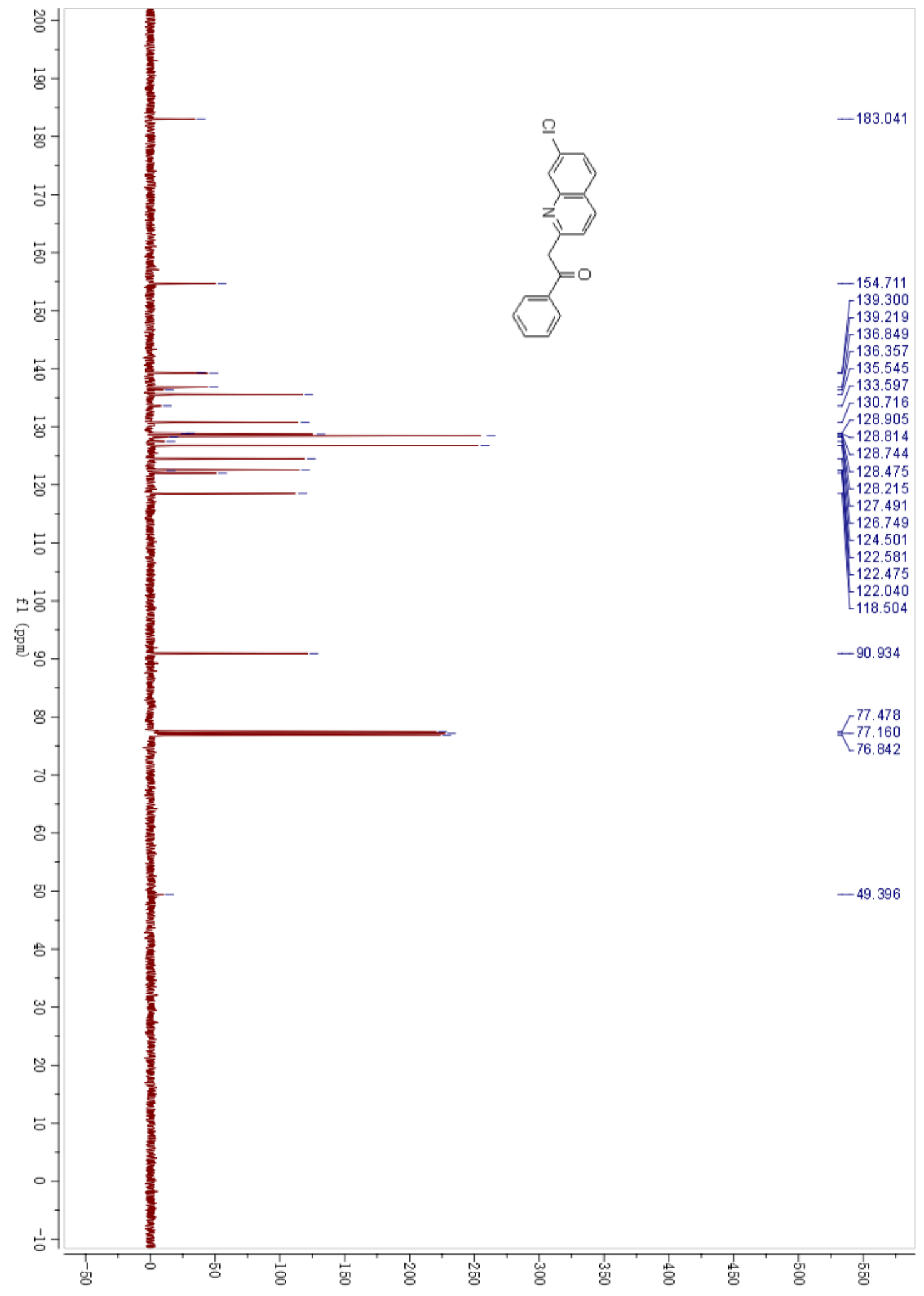


${ }^{1} \mathrm{H}$ NMR spectra of $\mathbf{2 a}$

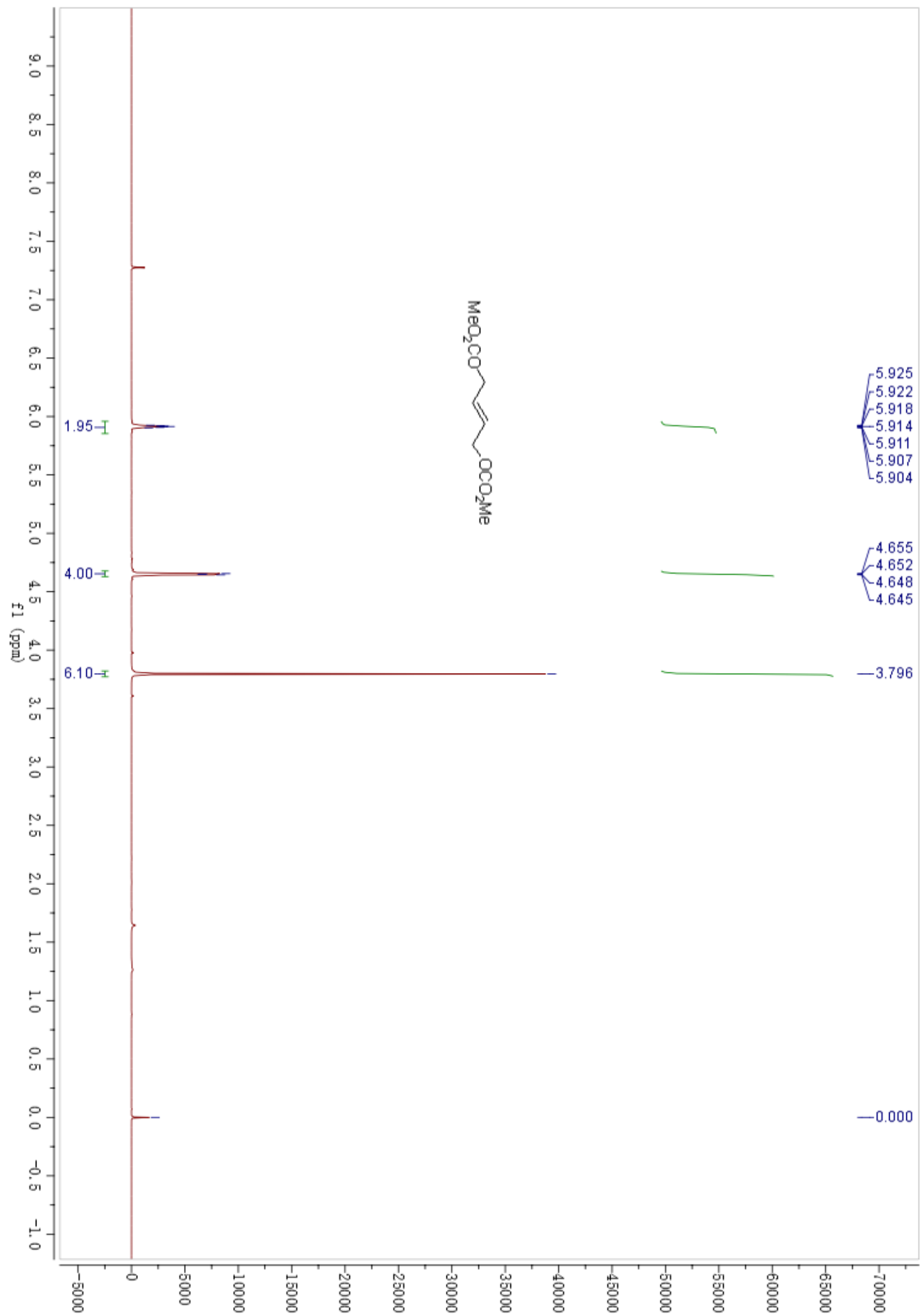


${ }^{1} \mathrm{H}$ NMR spectra of $\mathbf{2 b}$

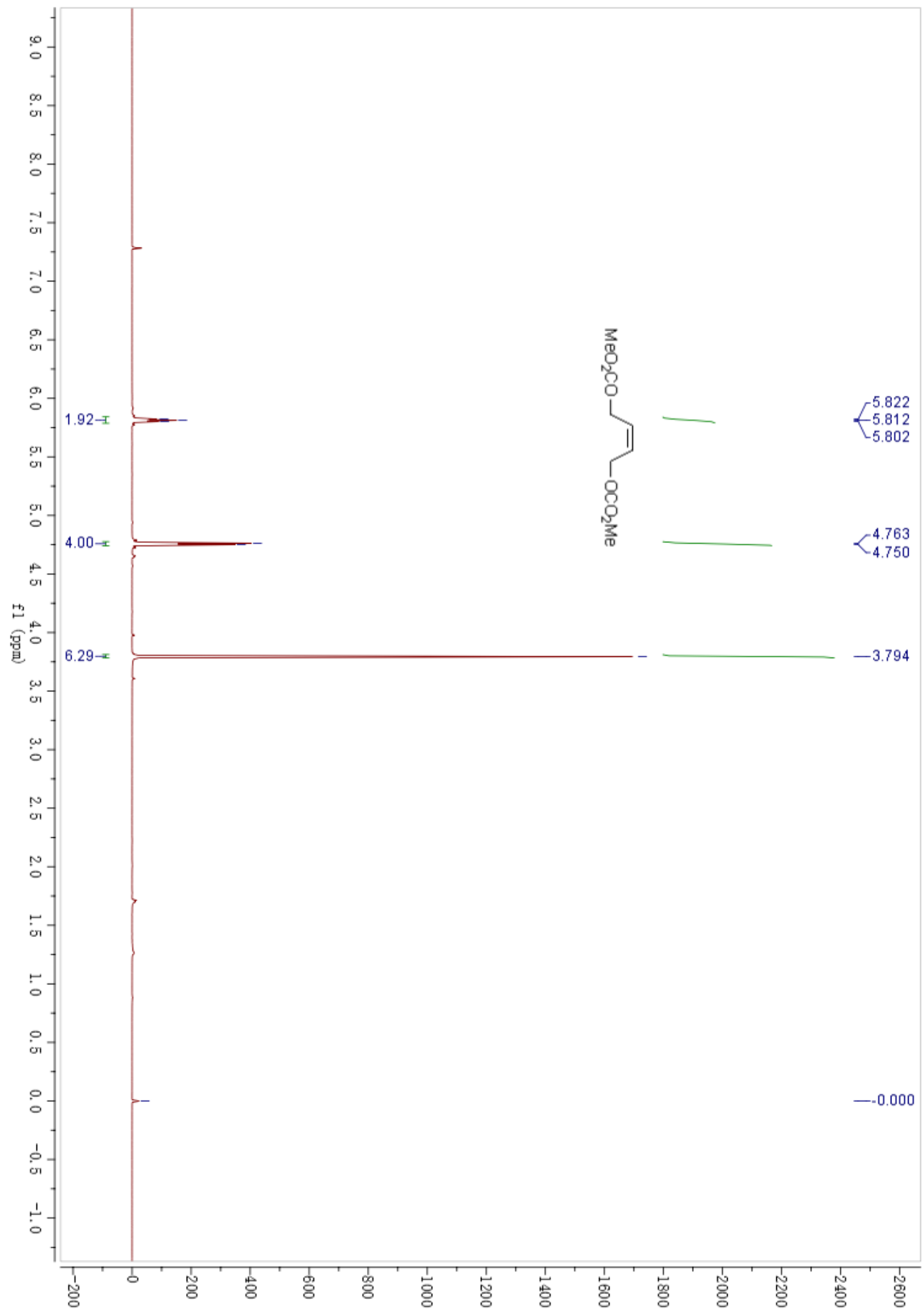


${ }^{1} \mathrm{H}$ NMR spectra of $\mathbf{2 c}$

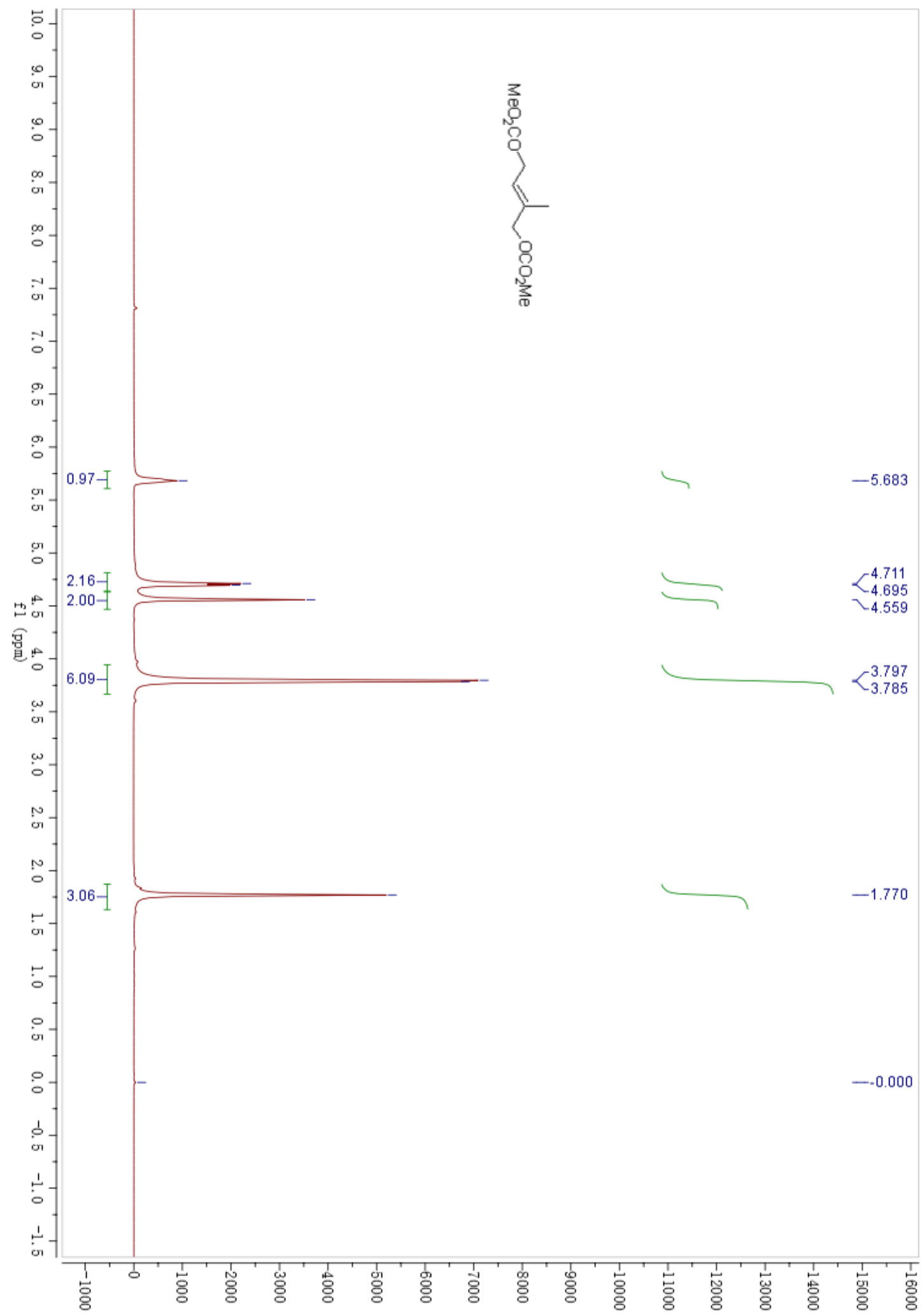


${ }^{13} \mathrm{C}$ NMR spectra of $\mathbf{2 c}$

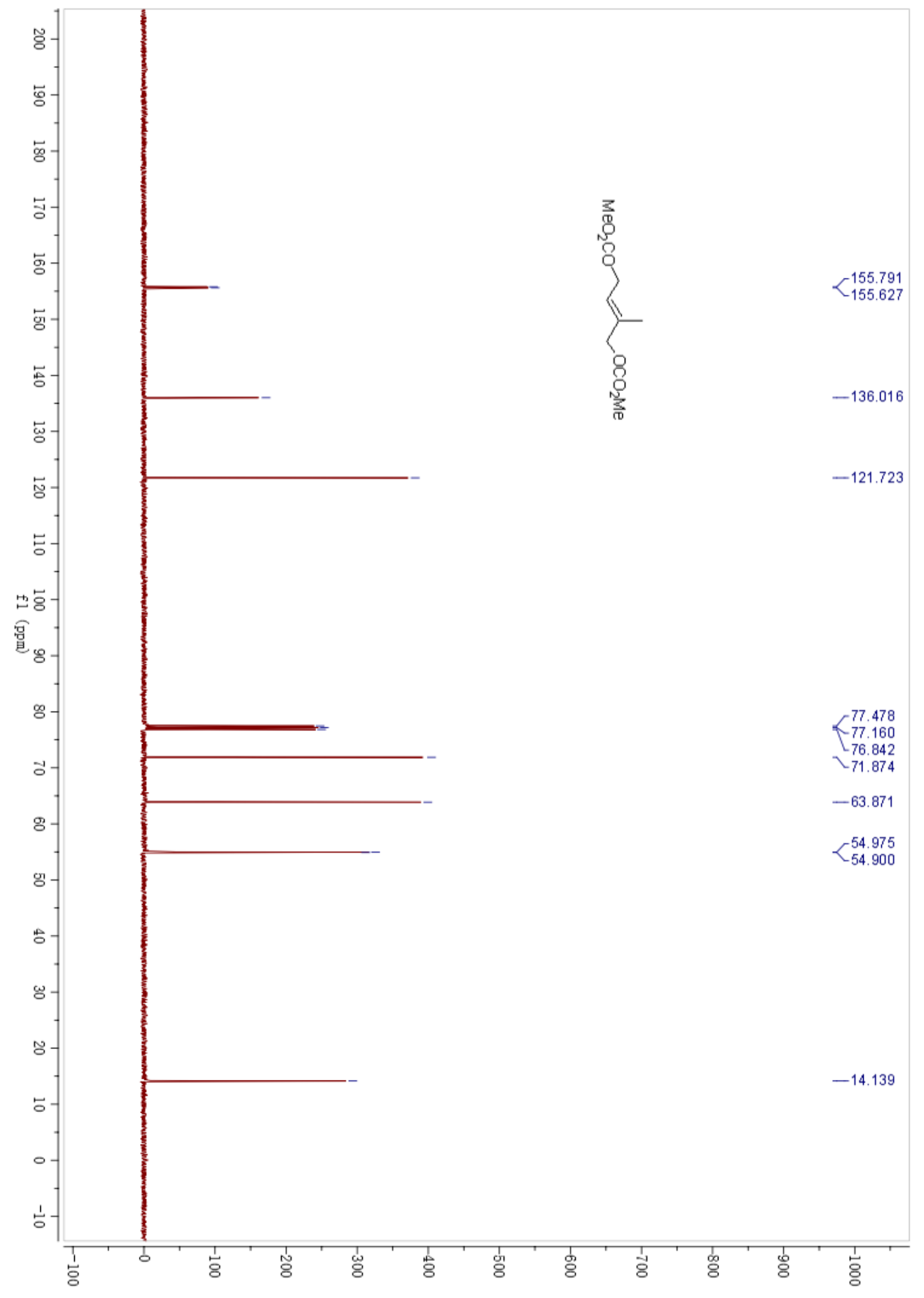


${ }^{1} \mathrm{H}$ NMR spectra of $\mathbf{3 a}$

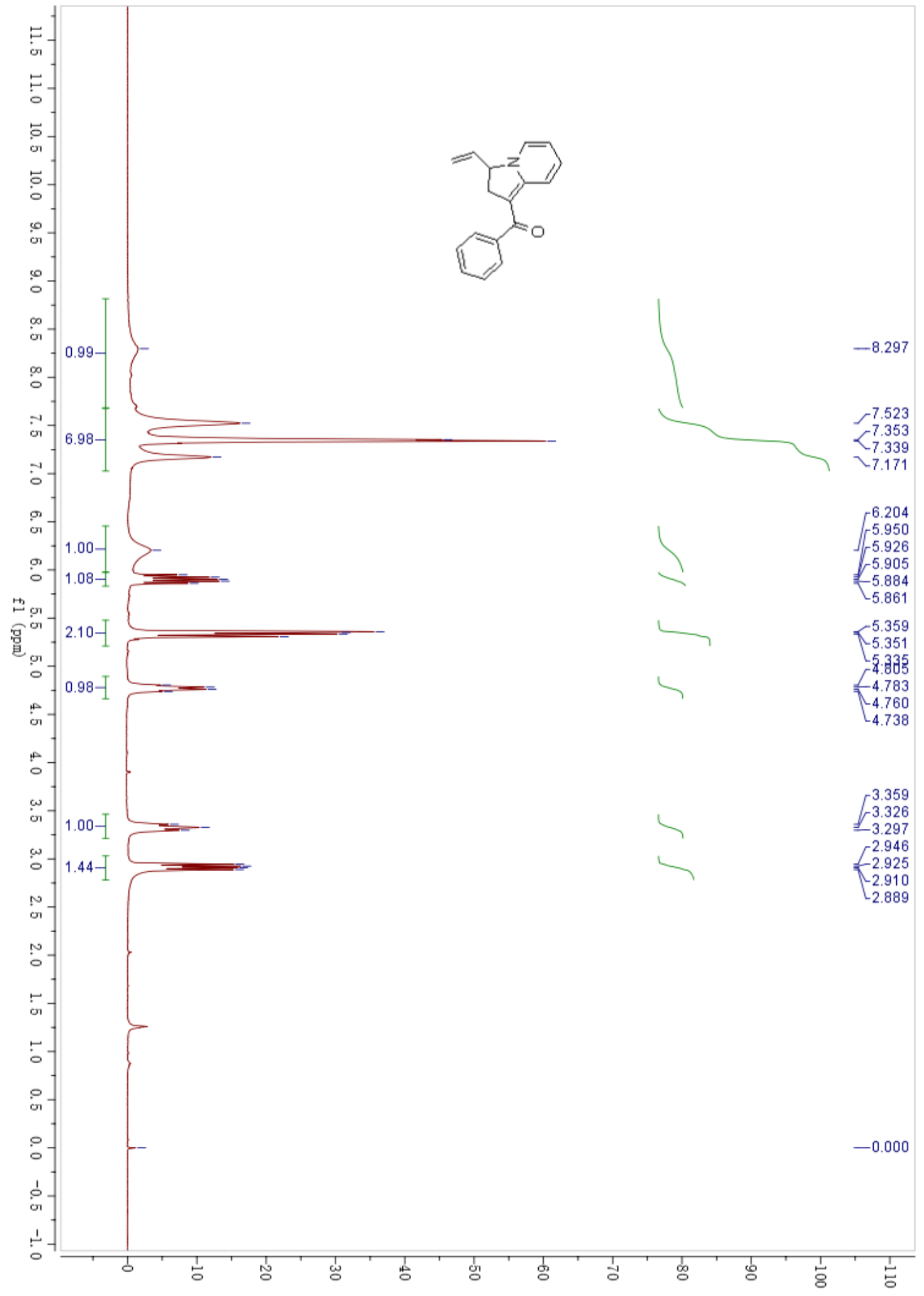


${ }^{1} \mathrm{H}$ NMR spectra of $\mathbf{3 b}$

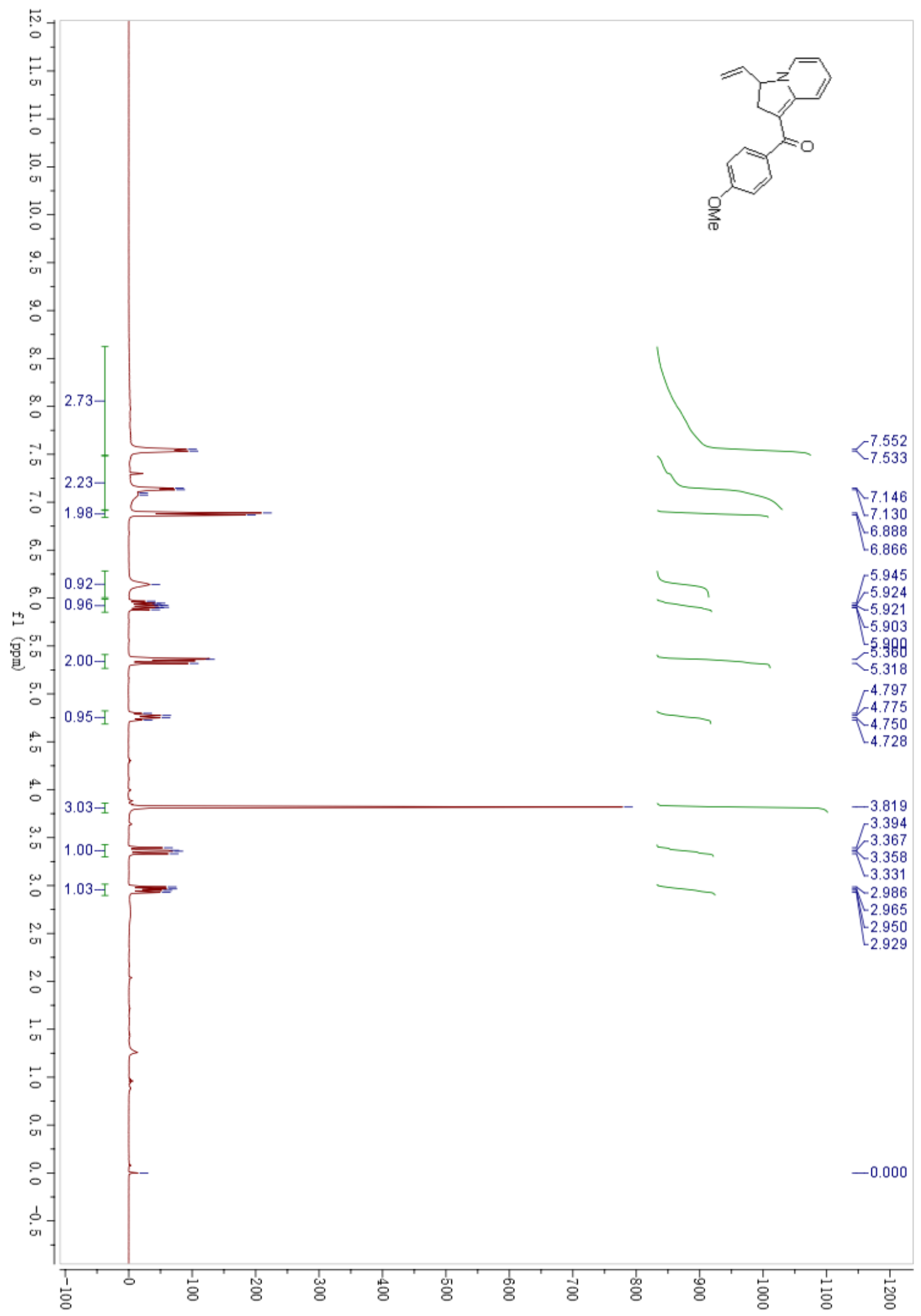


${ }^{13} \mathrm{C}$ NMR spectra of $\mathbf{3 b}$

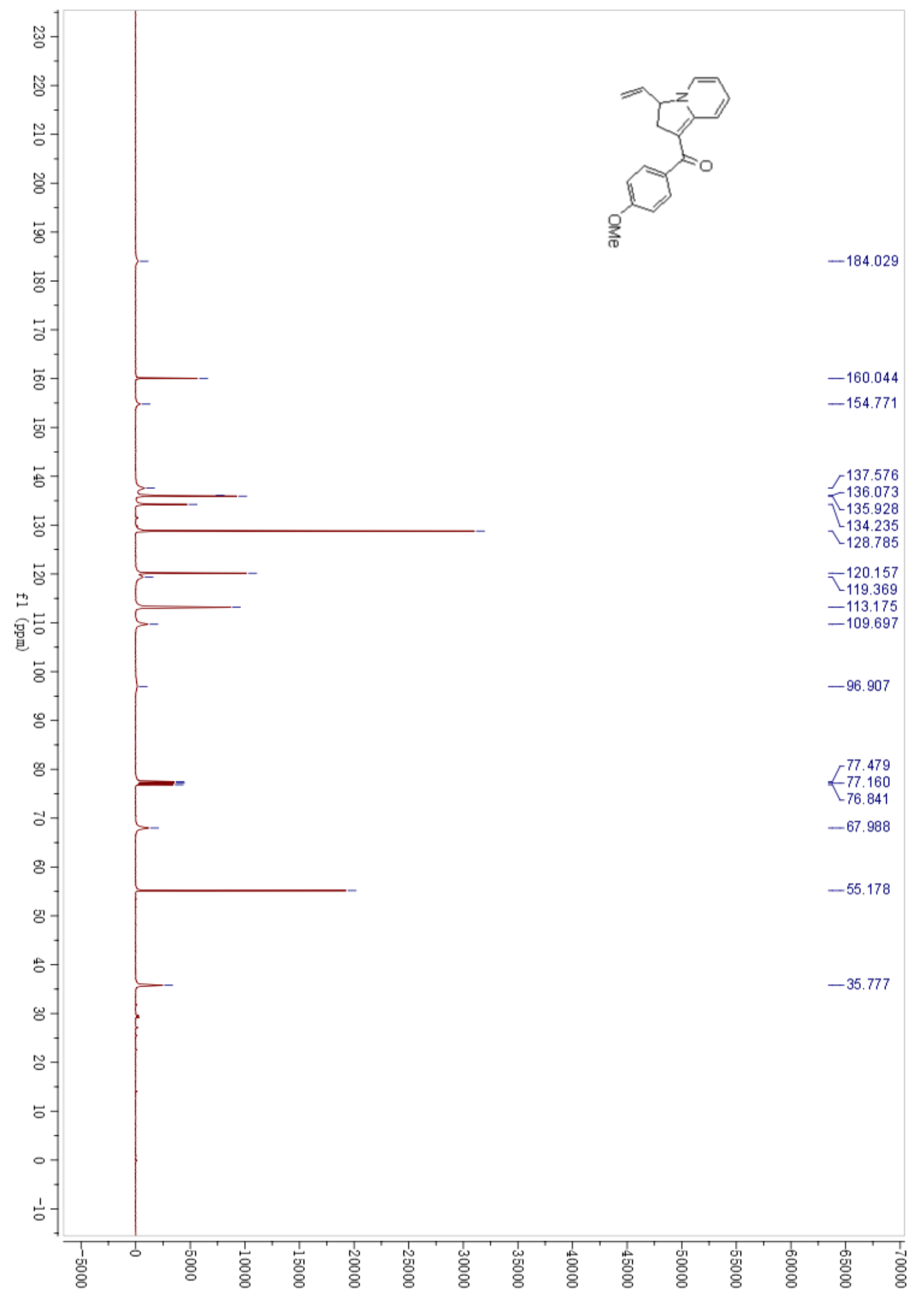


${ }^{1} \mathrm{H}$ NMR spectra of $\mathbf{3 c}$

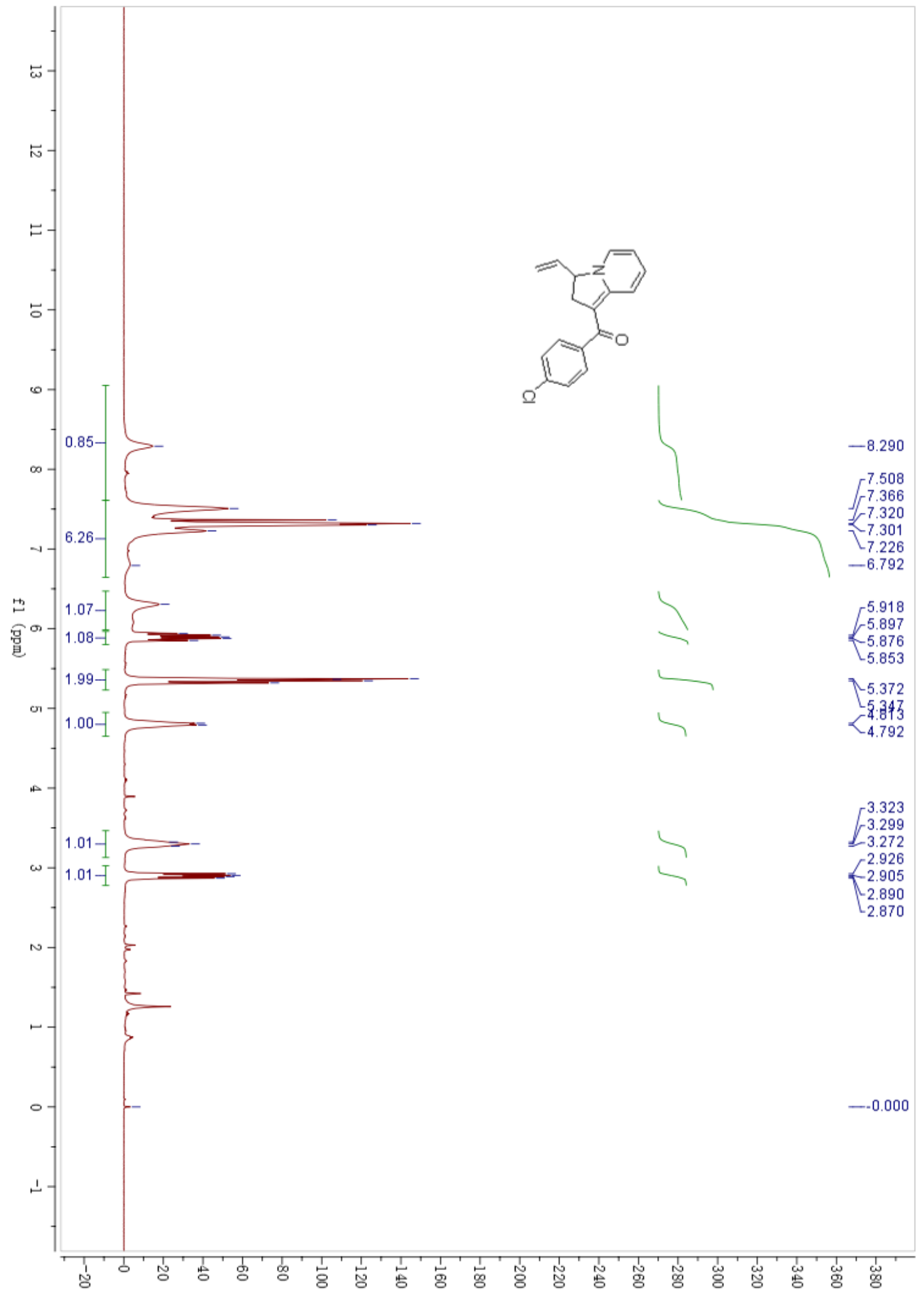


${ }^{13} \mathrm{C}$ NMR spectra of $\mathbf{3 c}$

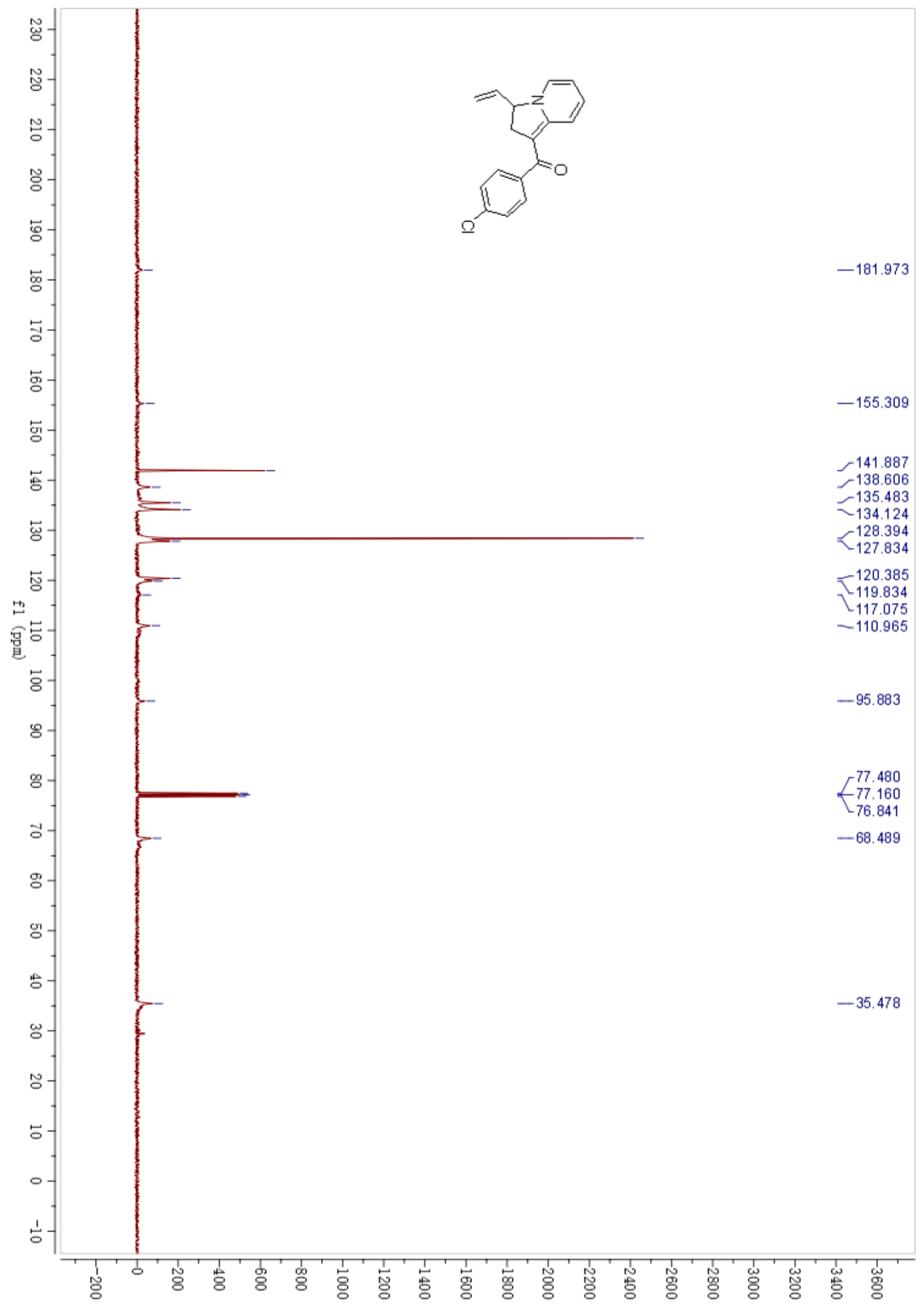


${ }^{1} \mathrm{H}$ NMR spectra of $\mathbf{3 d}$

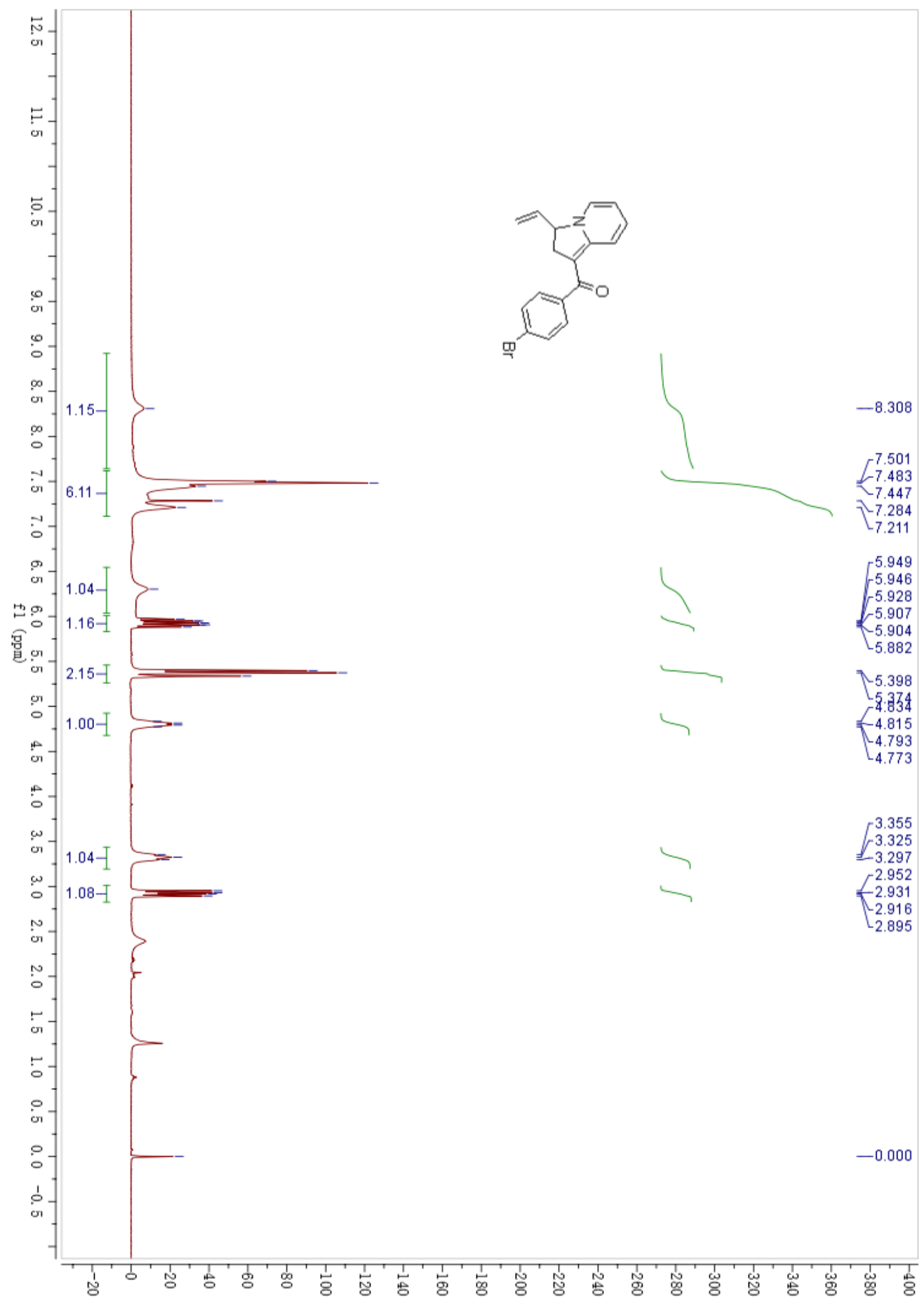


${ }^{1} \mathrm{H}$ NMR spectra of $\mathbf{3 e}$

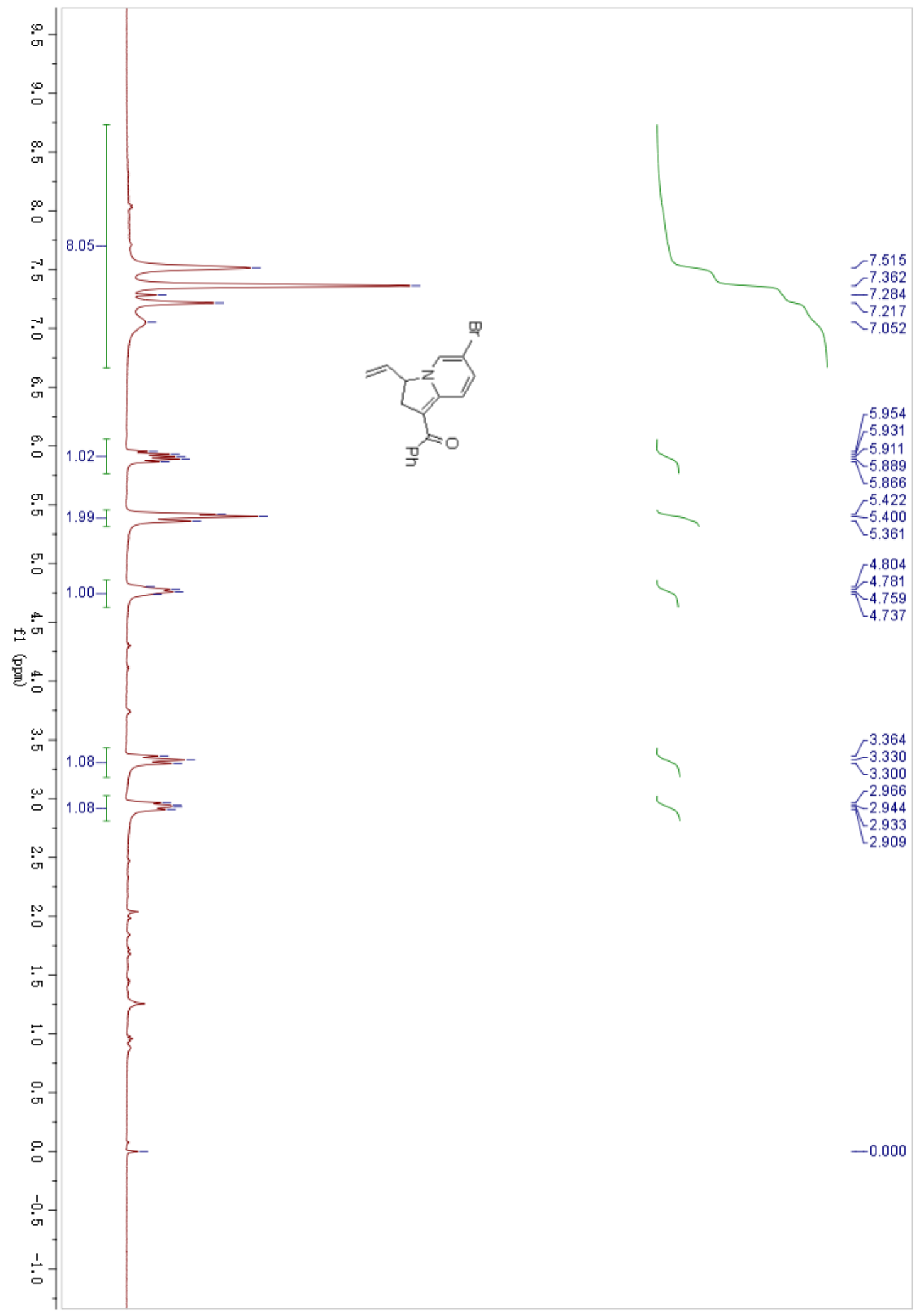


${ }^{13} \mathrm{C}$ NMR spectra of $\mathbf{3 e}$

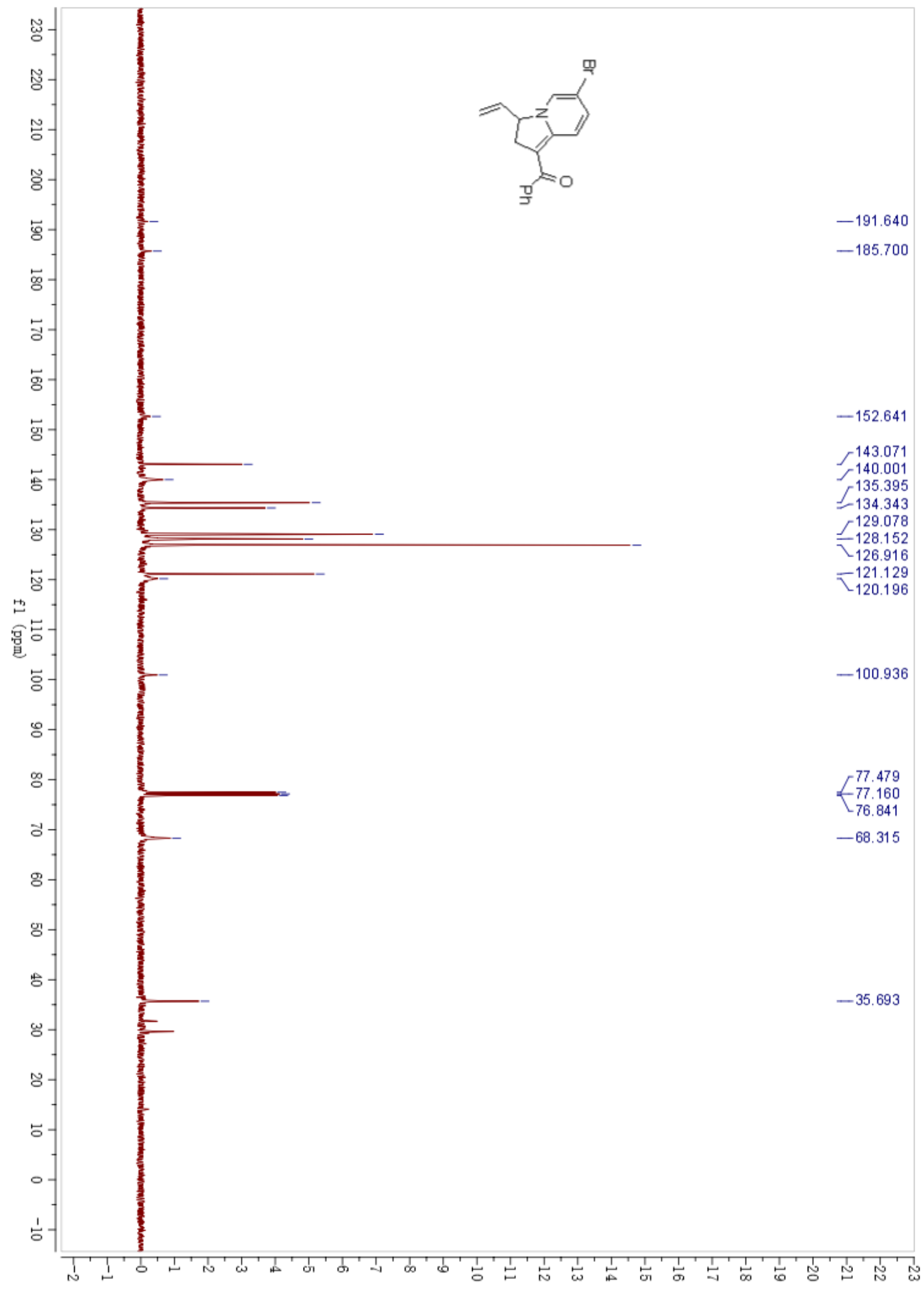


${ }^{1} \mathrm{H}$ NMR spectra of $\mathbf{3 f}$

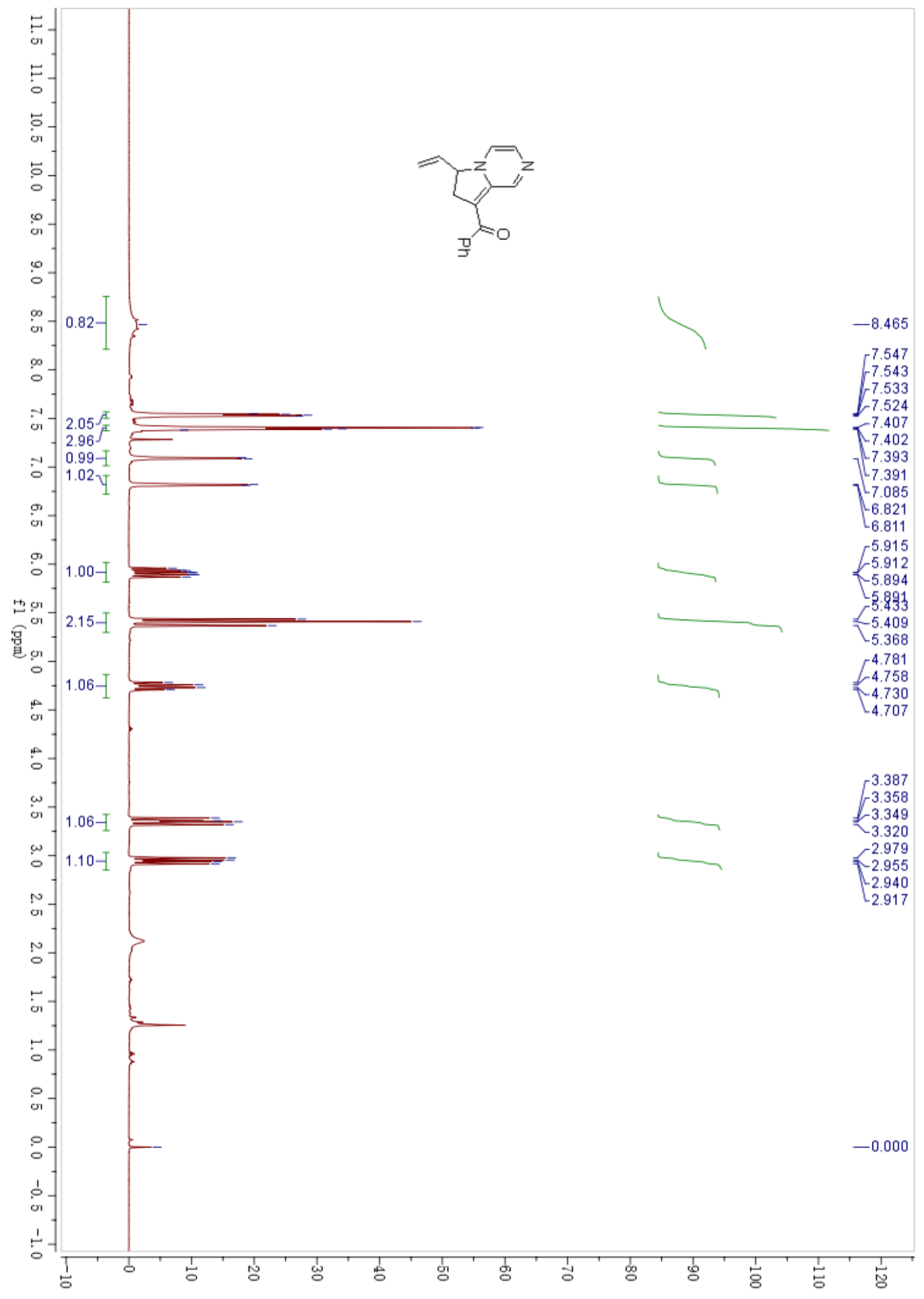


${ }^{1} \mathrm{H}$ NMR spectra of $\mathbf{3 g}$

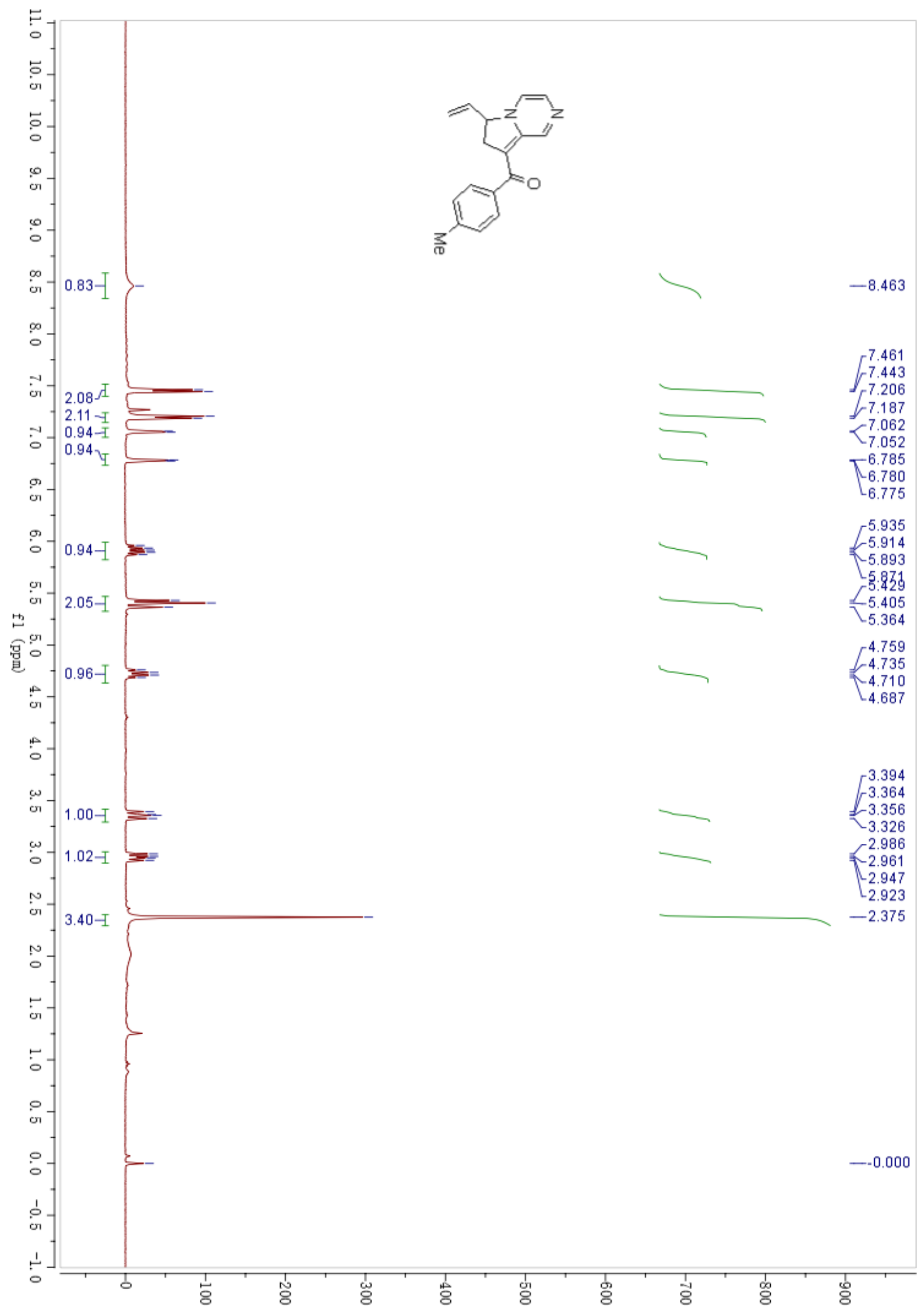


${ }^{1} \mathrm{H}$ NMR spectra of $\mathbf{3 h}$

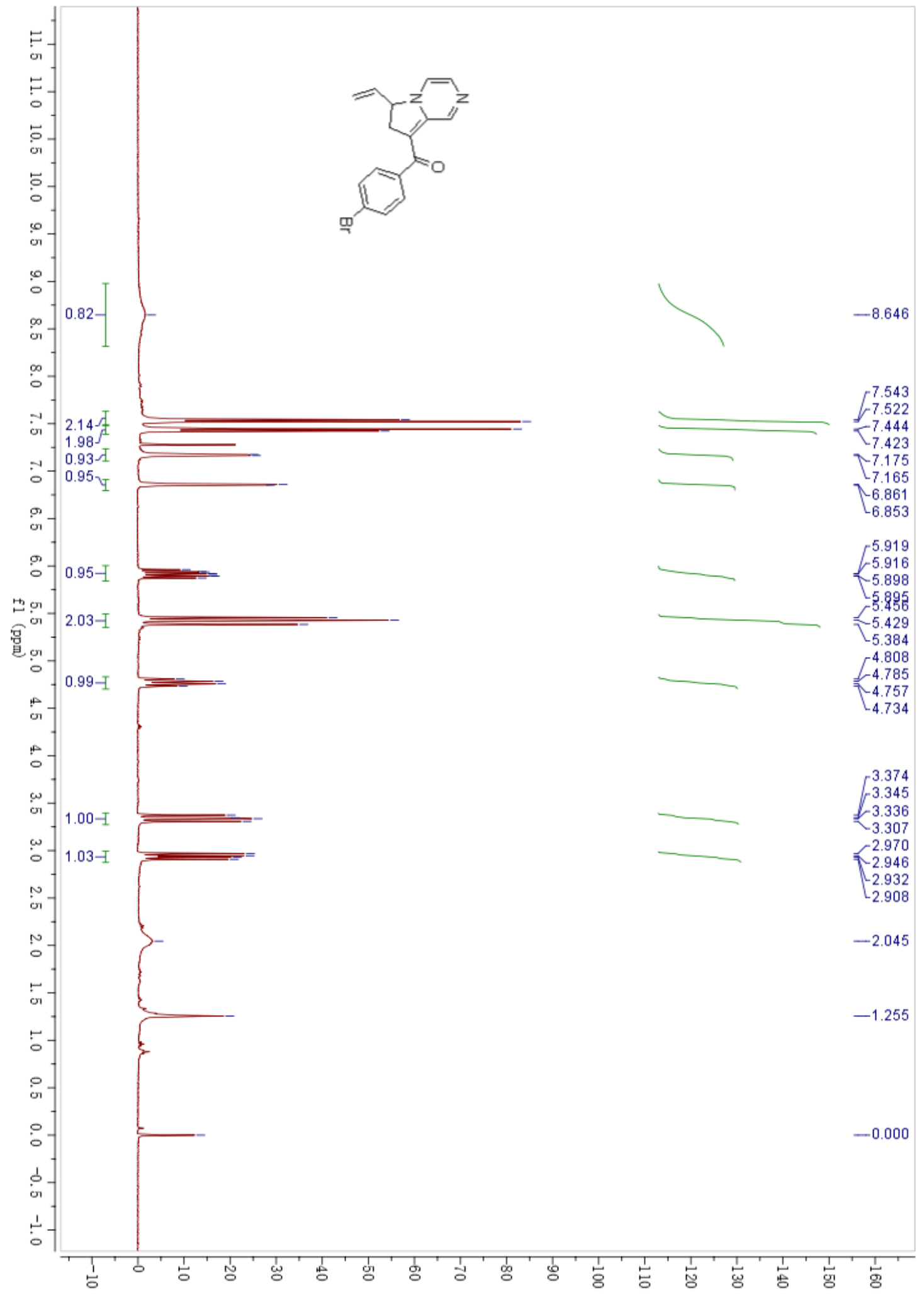


${ }^{1} \mathrm{H}$ NMR spectra of $\mathbf{3} \mathbf{h}$ '

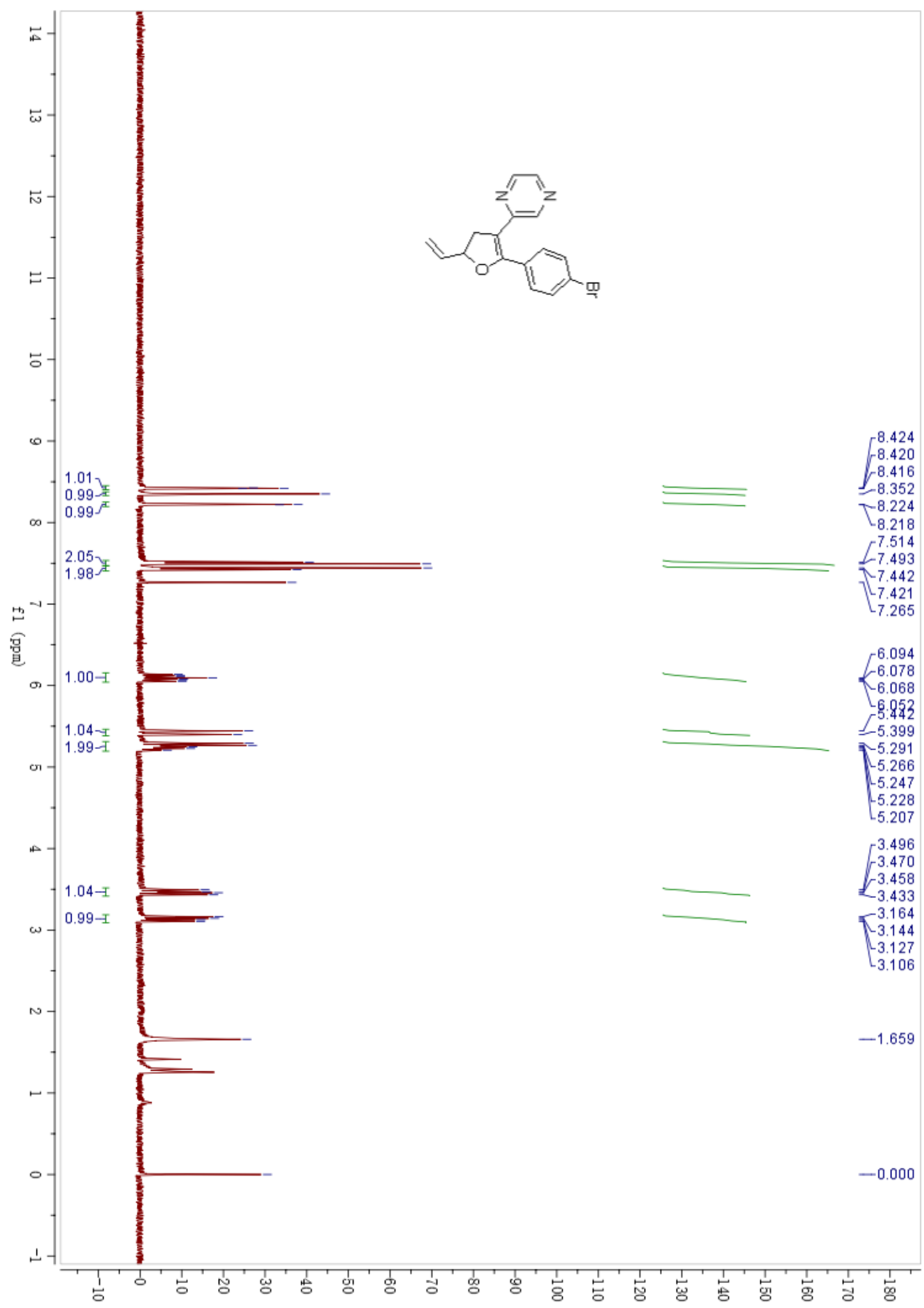


${ }^{13} \mathrm{C}$ NMR spectra of $\mathbf{3 h}$ '

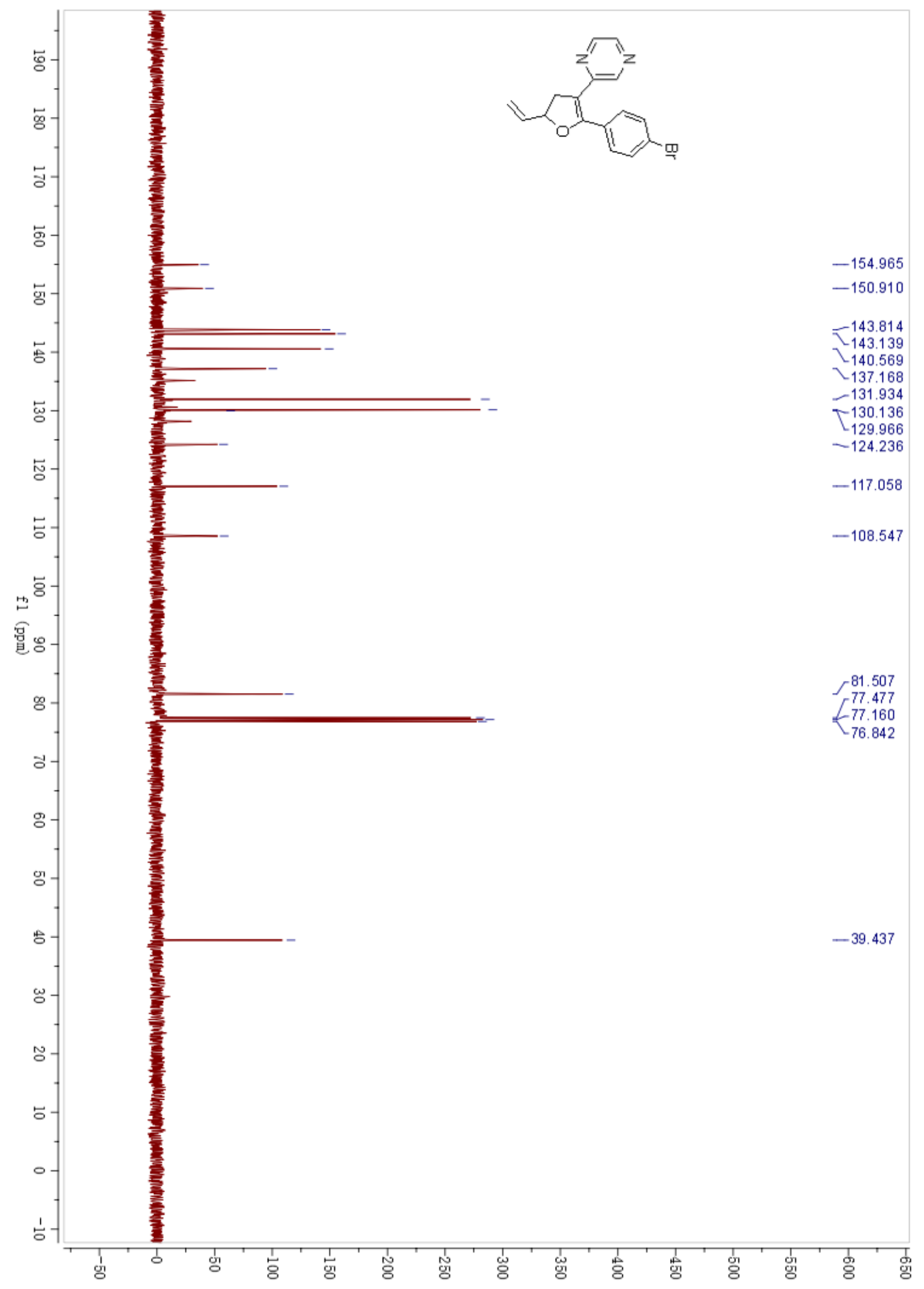


${ }^{1}$ H NMR spectra of $\mathbf{3 i}$

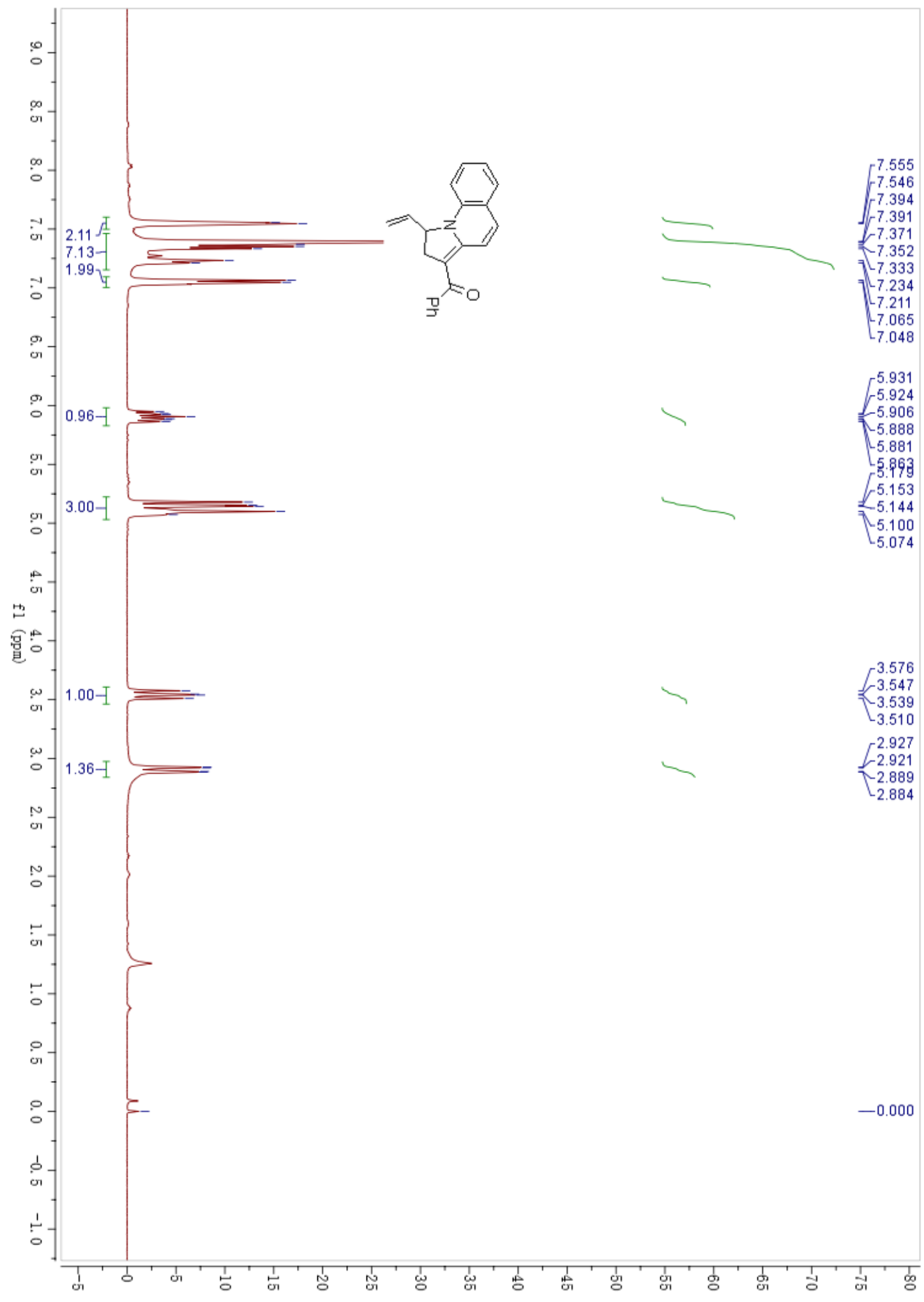


${ }^{1}$ H NMR spectra of $\mathbf{3 j}$

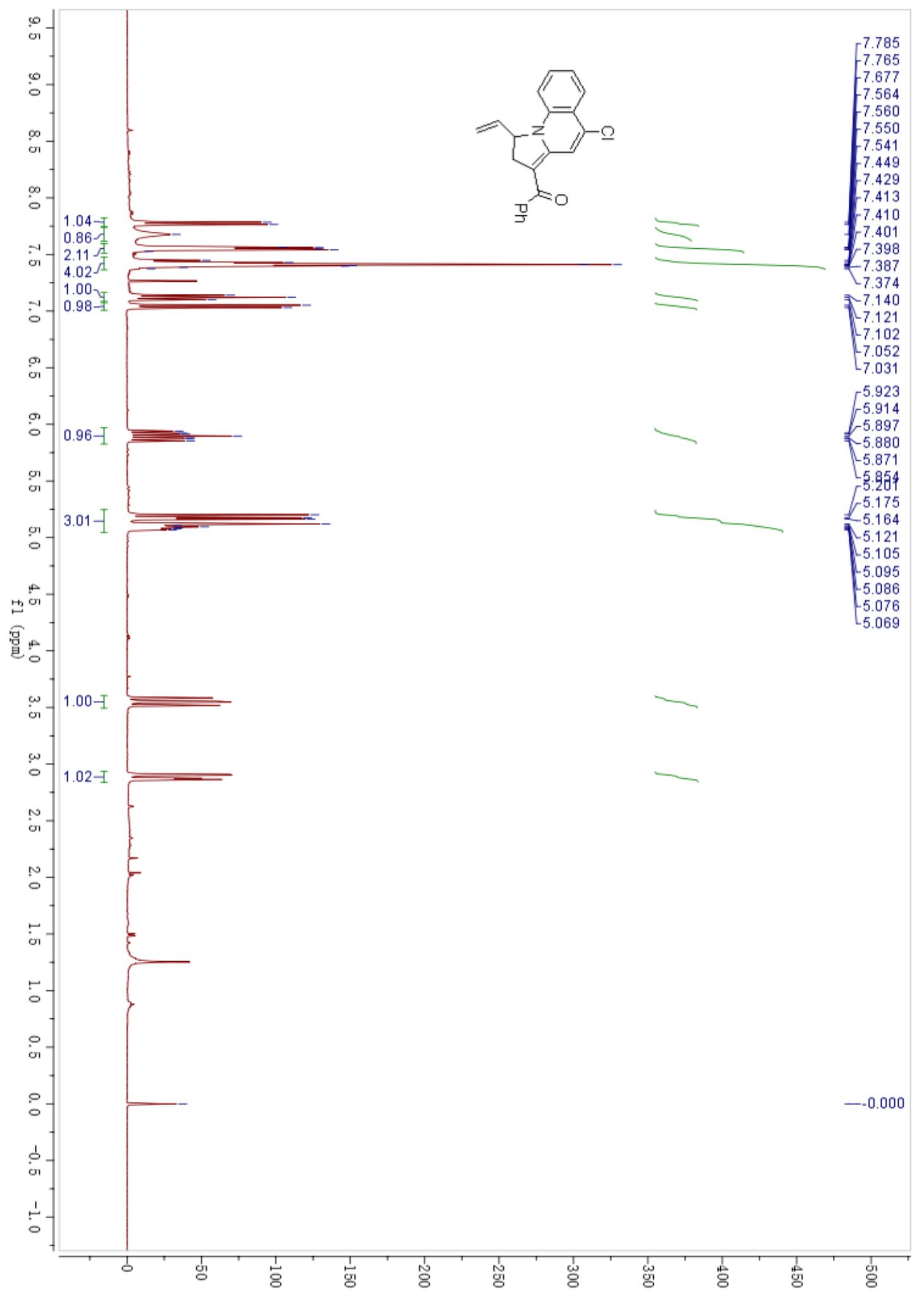


${ }^{13} \mathrm{C}$ NMR spectra of $\mathbf{3 j}$

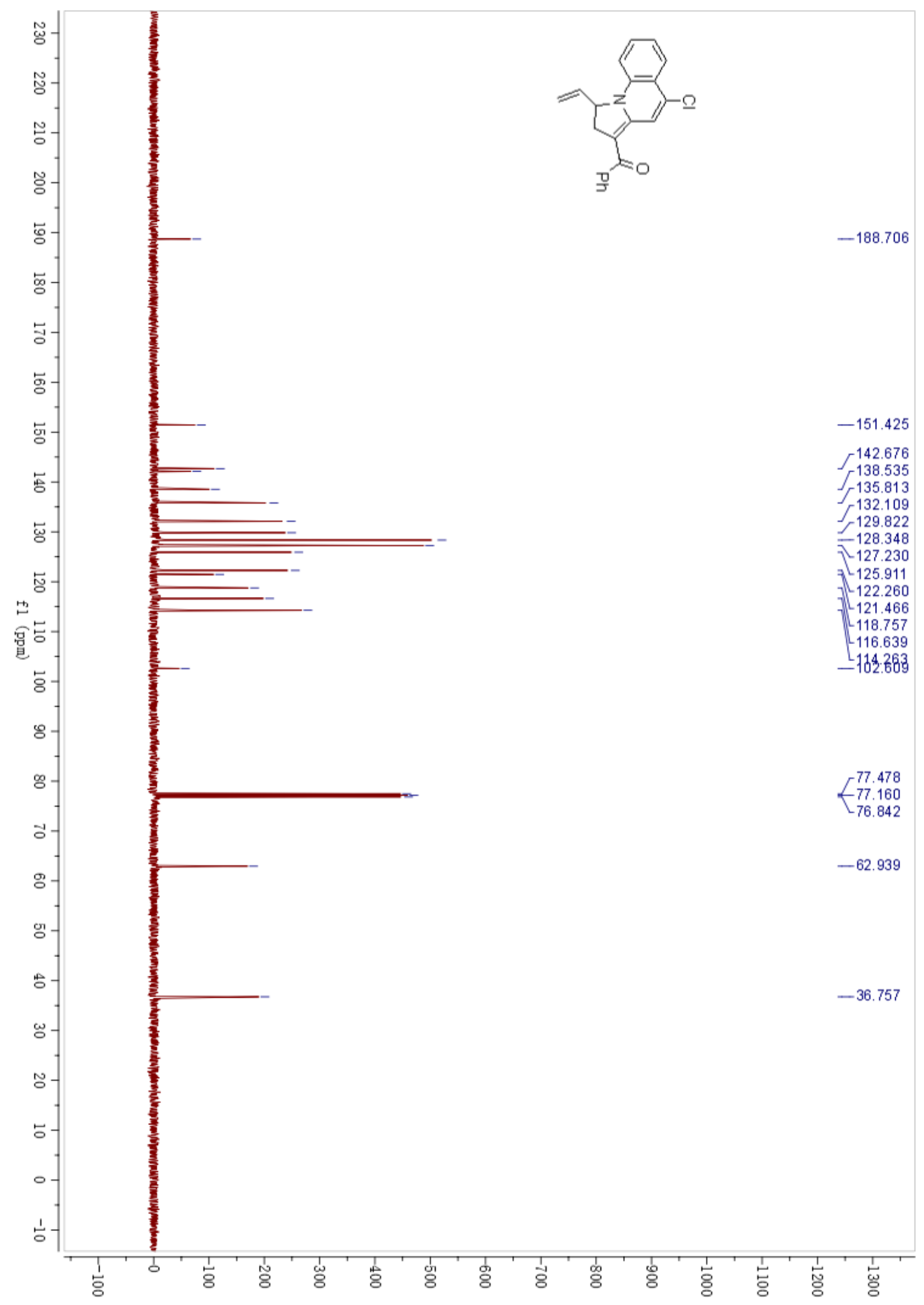


${ }^{1} \mathrm{H}$ NMR spectra of $\mathbf{3 k}$

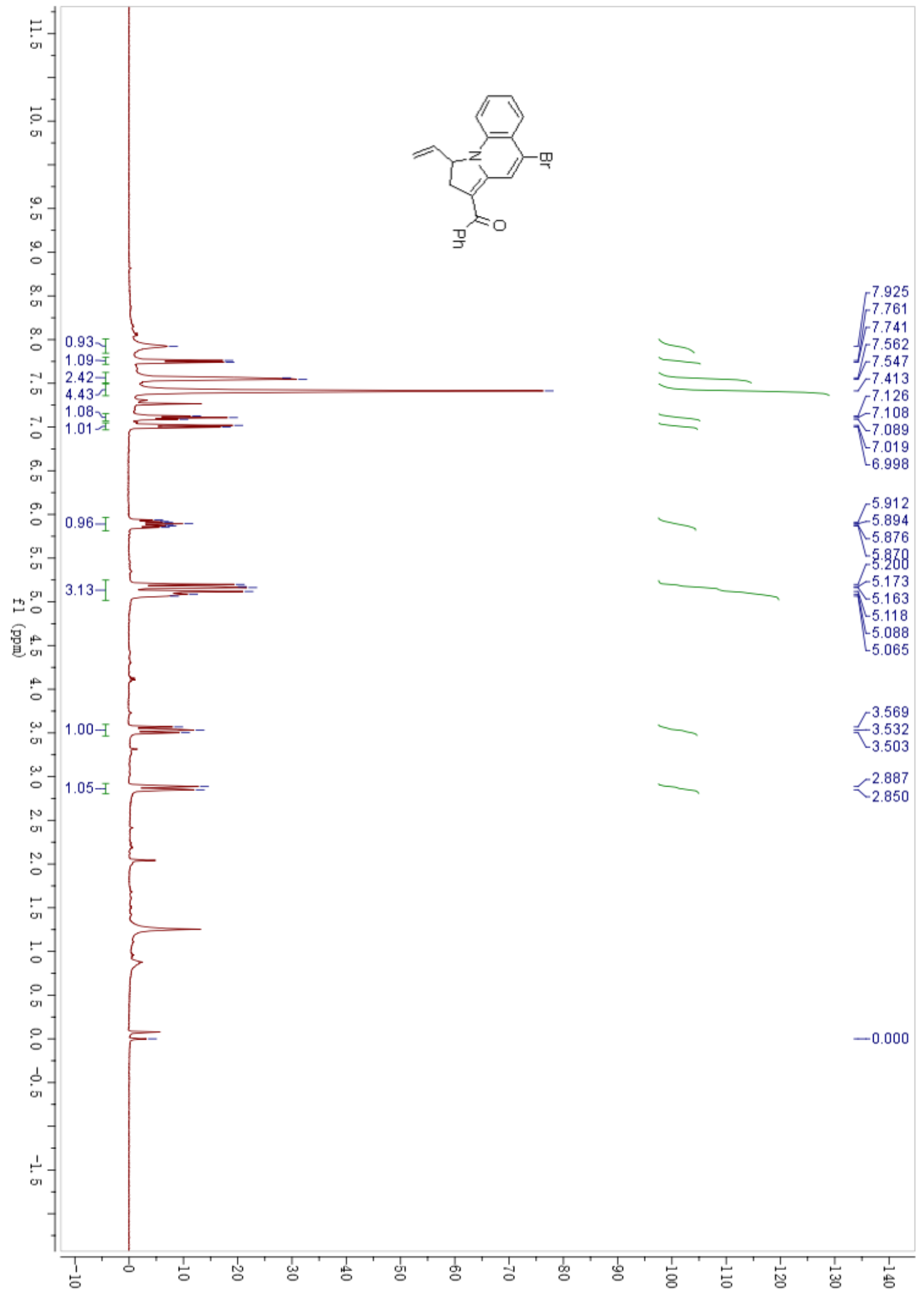


${ }^{13} \mathrm{C}$ NMR spectra of $\mathbf{3 k}$

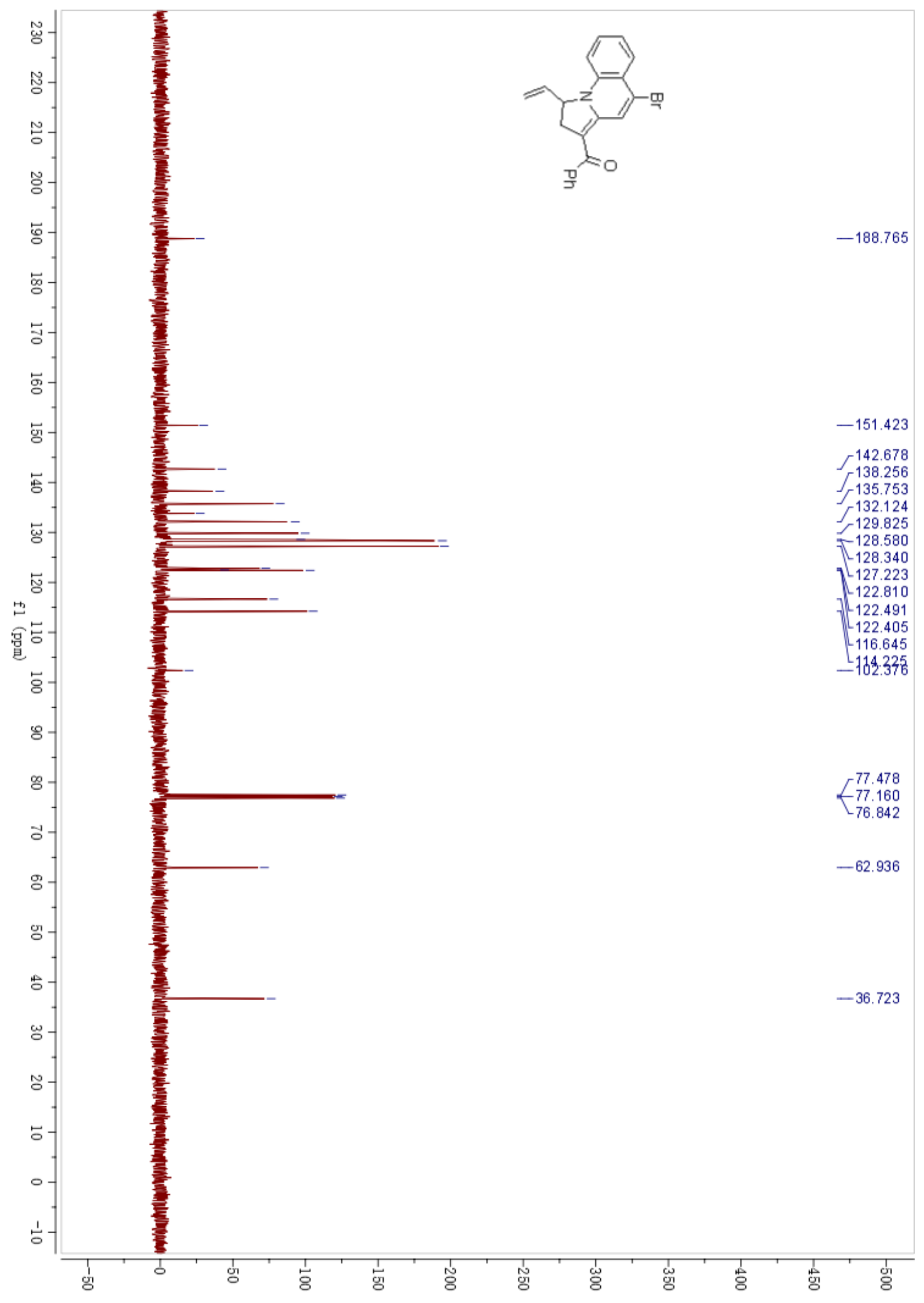


${ }^{1}$ H NMR spectra of 31

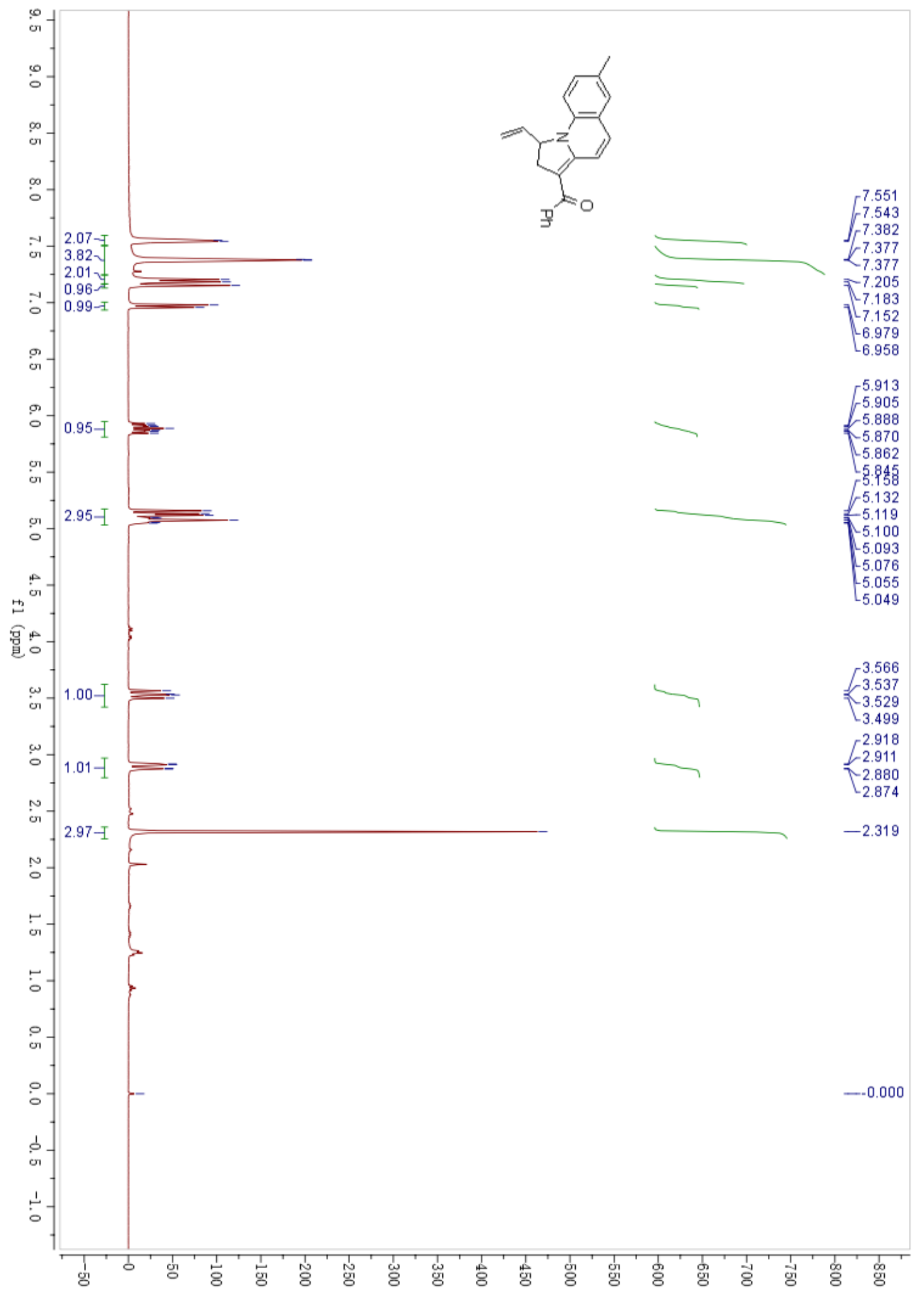


${ }^{13} \mathrm{C}$ NMR spectra of $\mathbf{3 l}$

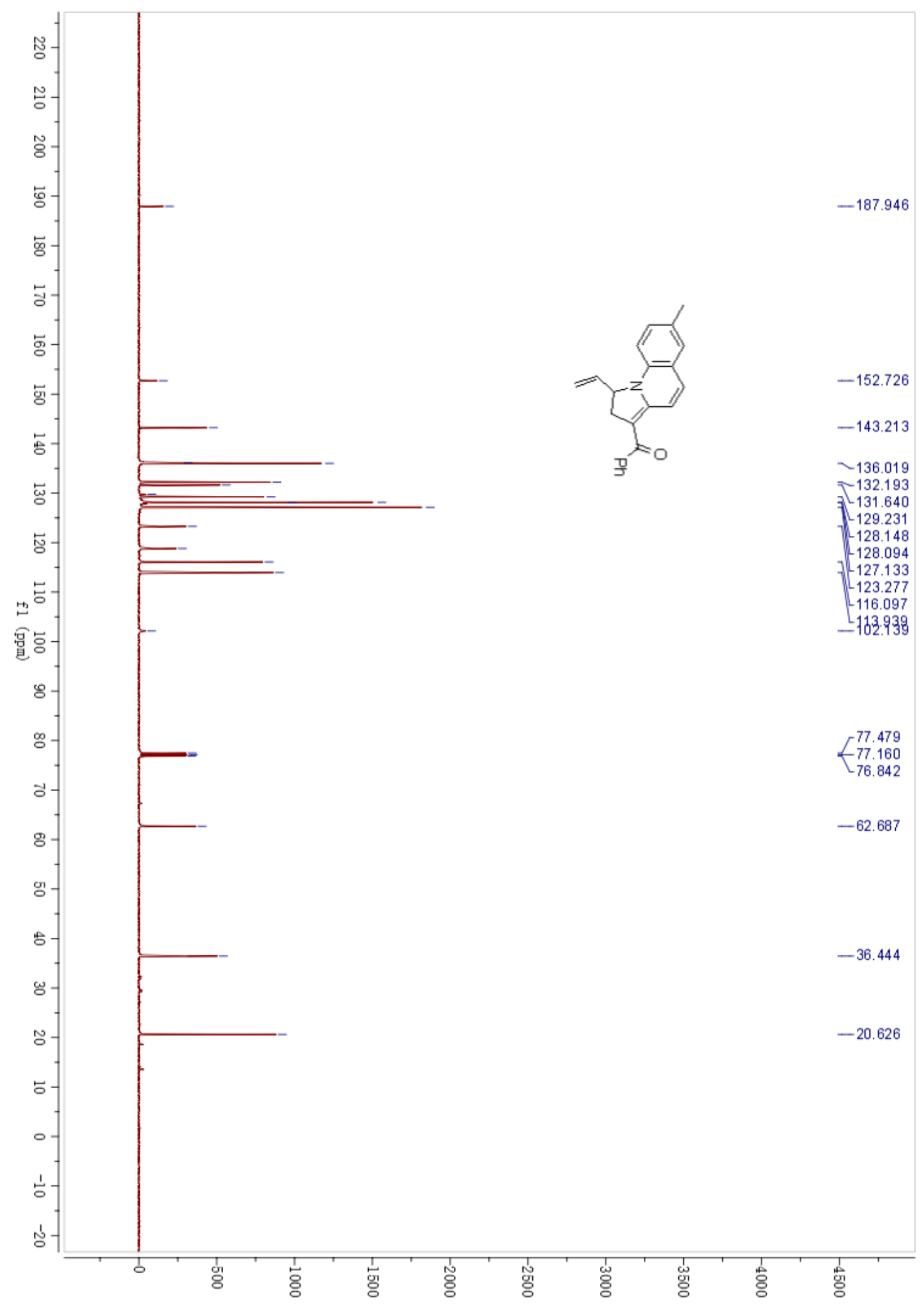


${ }^{1} \mathrm{H}$ NMR spectra of $\mathbf{3 m}$

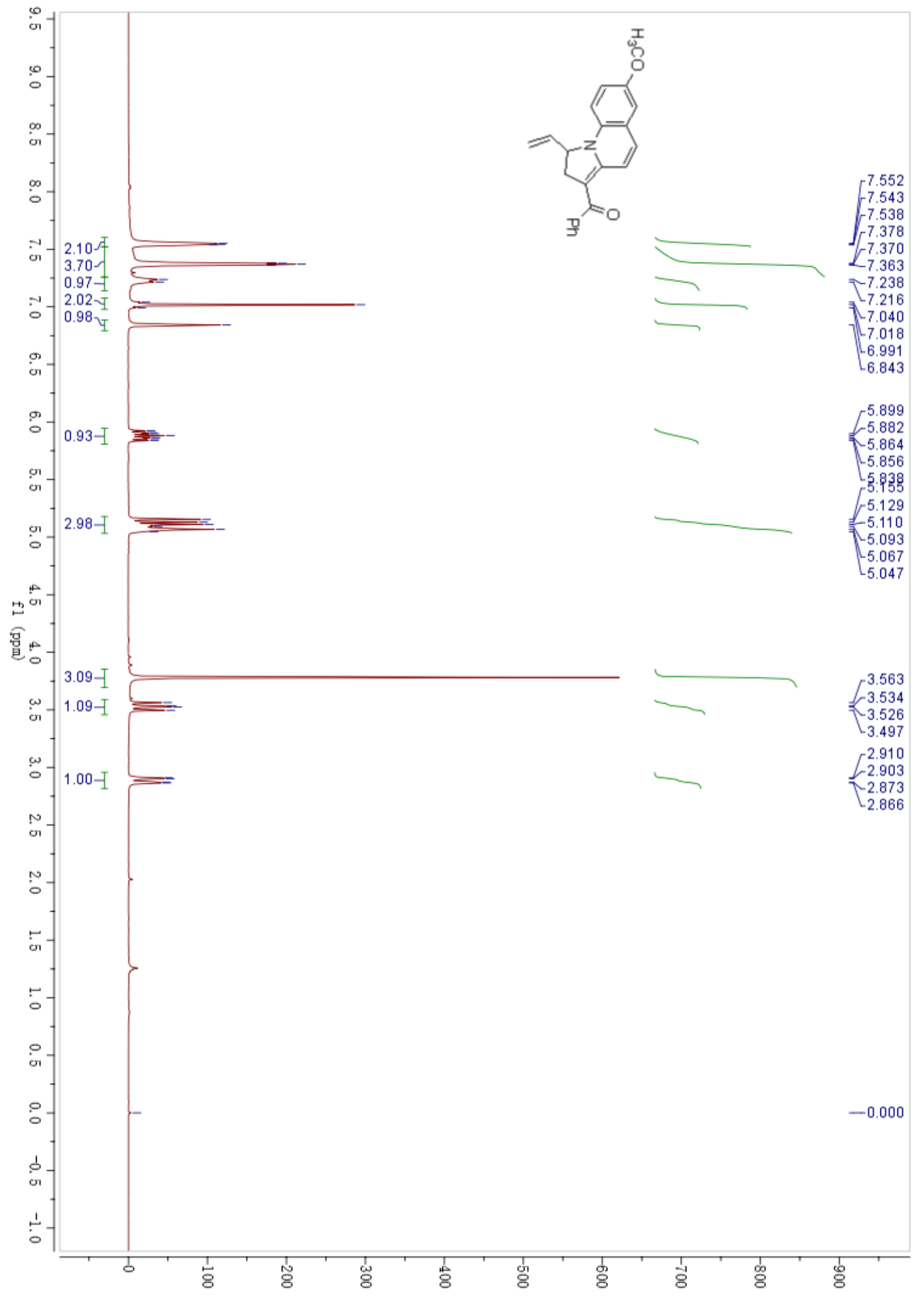


${ }^{13} \mathrm{C}$ NMR spectra of $\mathbf{3 m}$

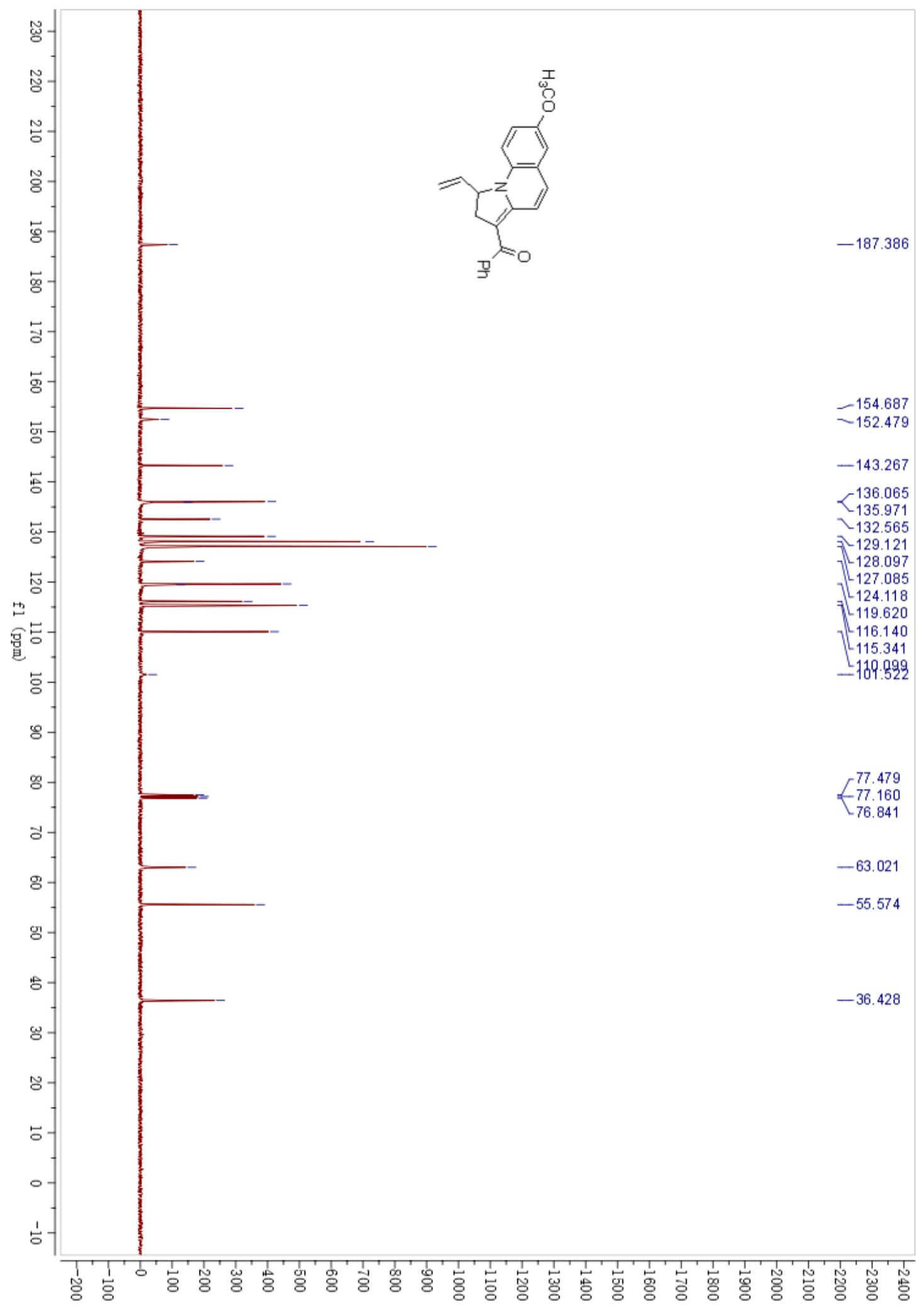


${ }^{1} \mathrm{H}$ NMR spectra of $\mathbf{3 n}$

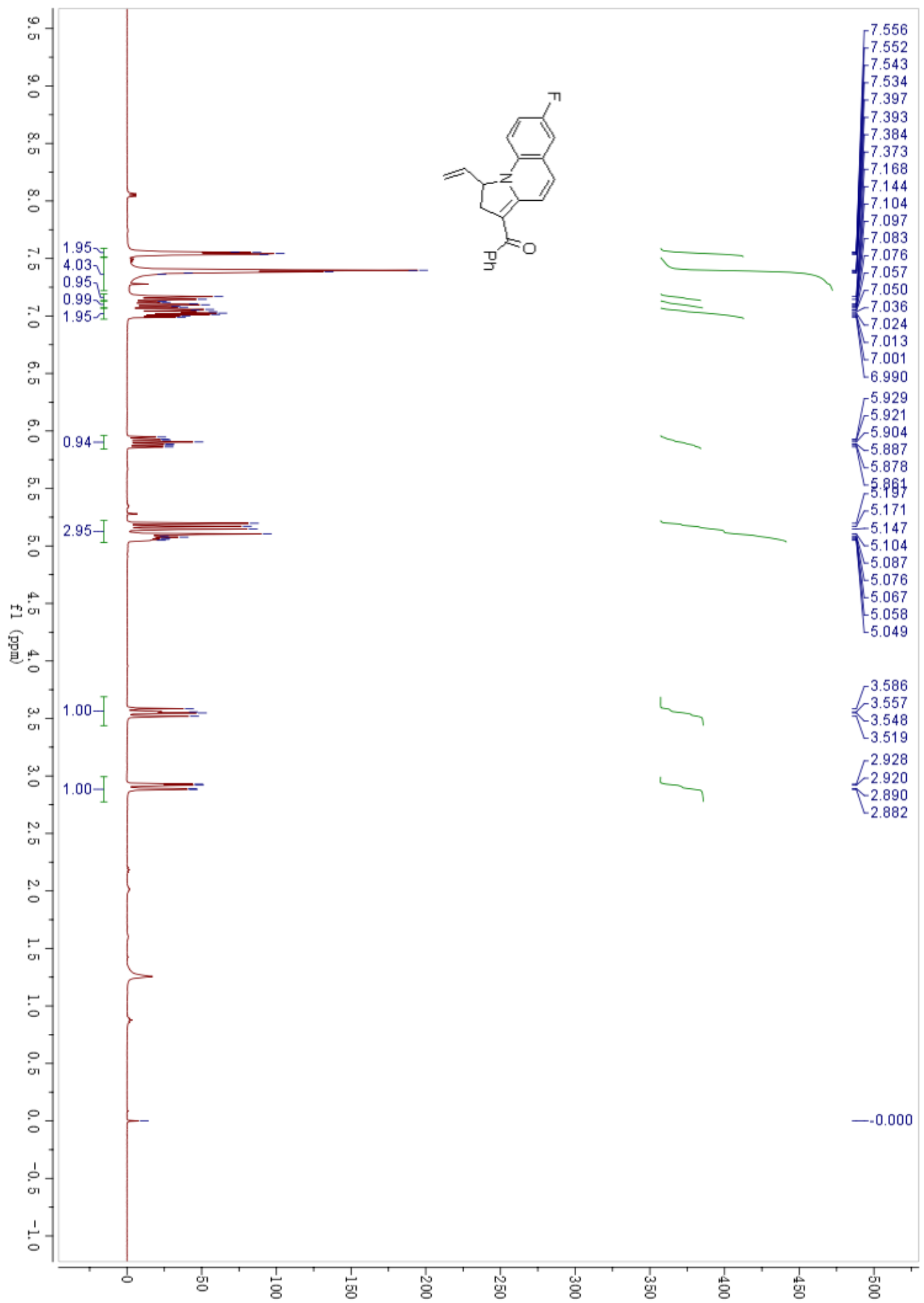


${ }^{13} \mathrm{C}$ NMR spectra of $\mathbf{3 n}$

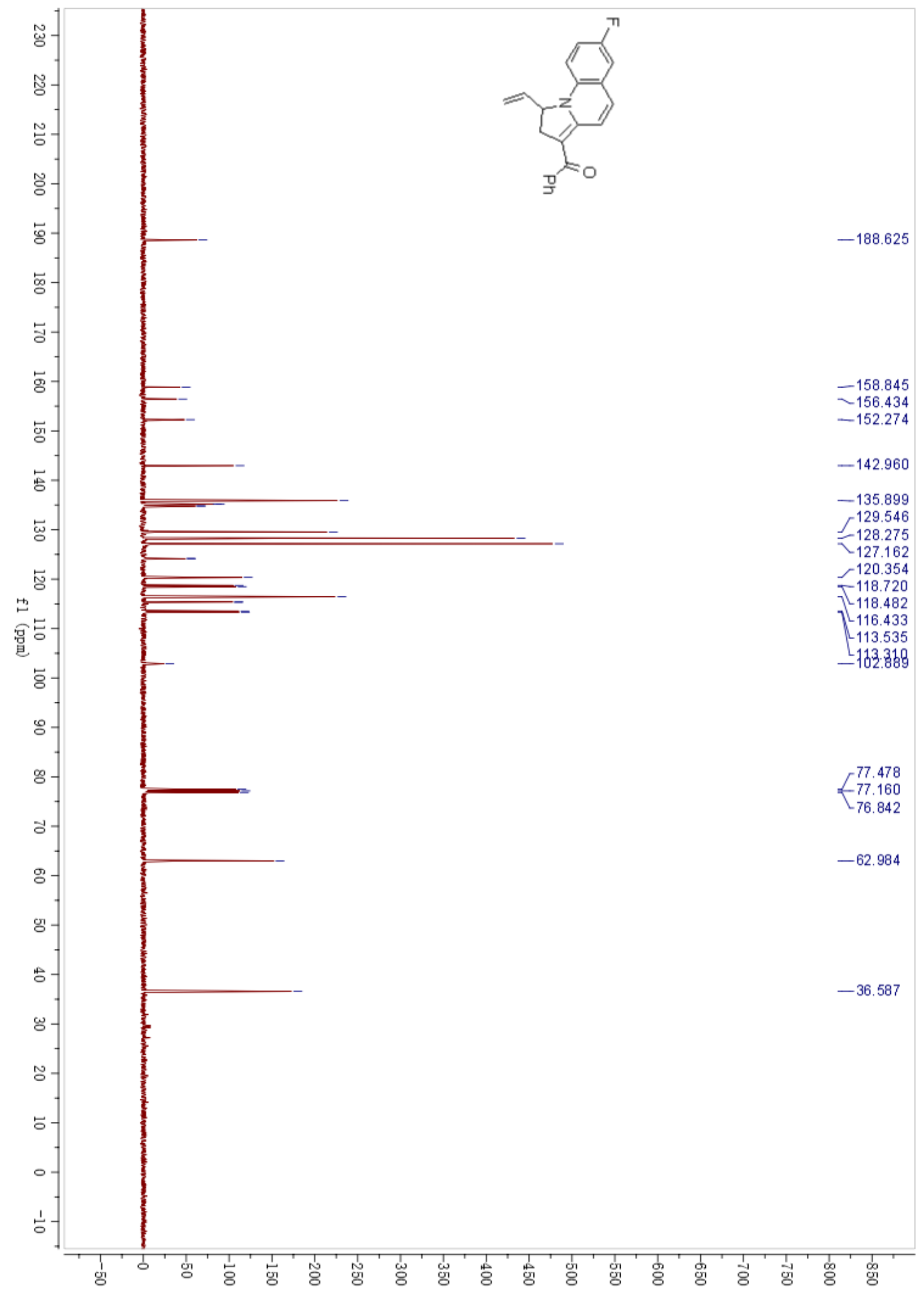


${ }^{19}$ F NMR spectra of $\mathbf{3 n}$

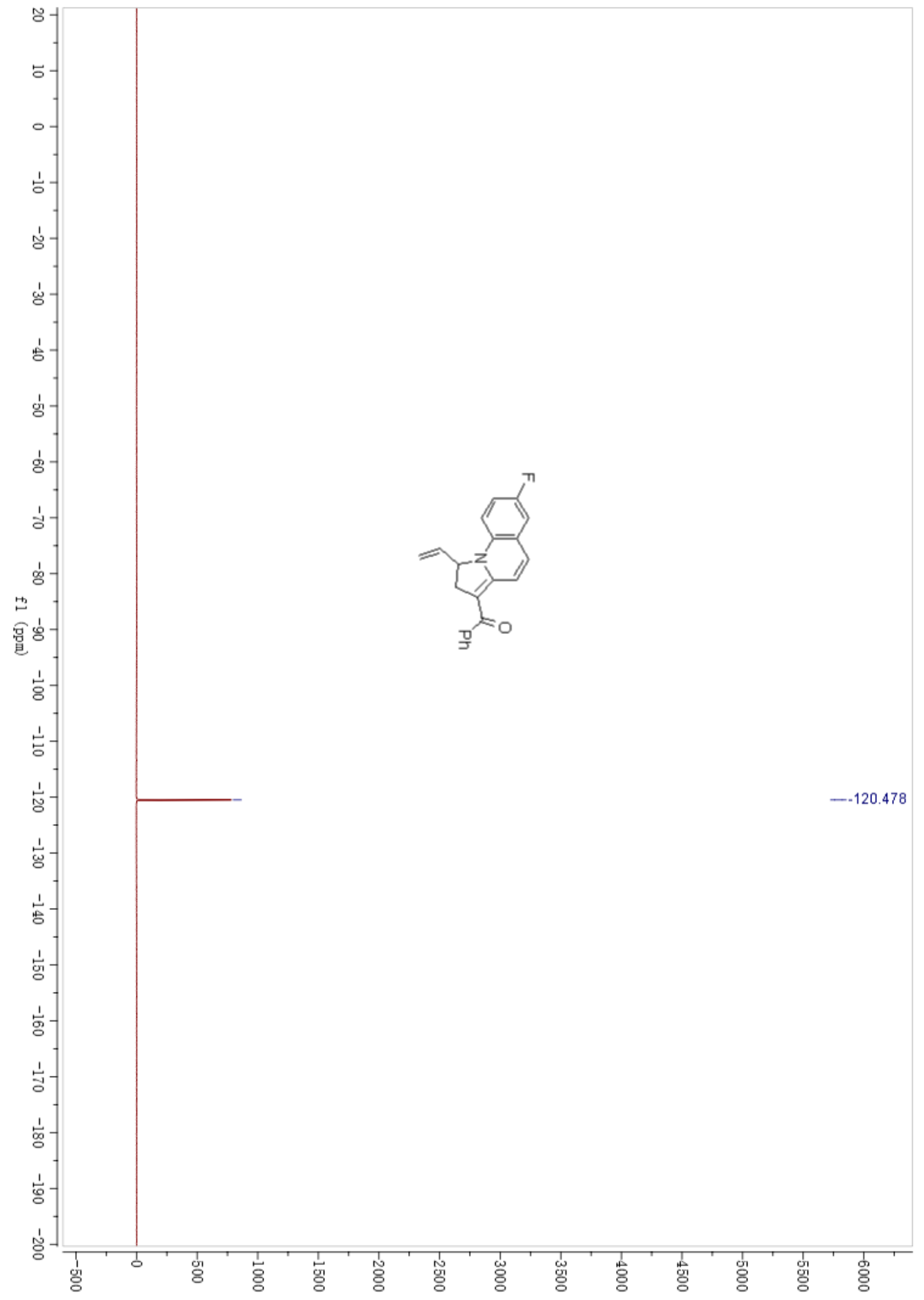


${ }^{1} \mathrm{H}$ NMR spectra of $\mathbf{3 o}$

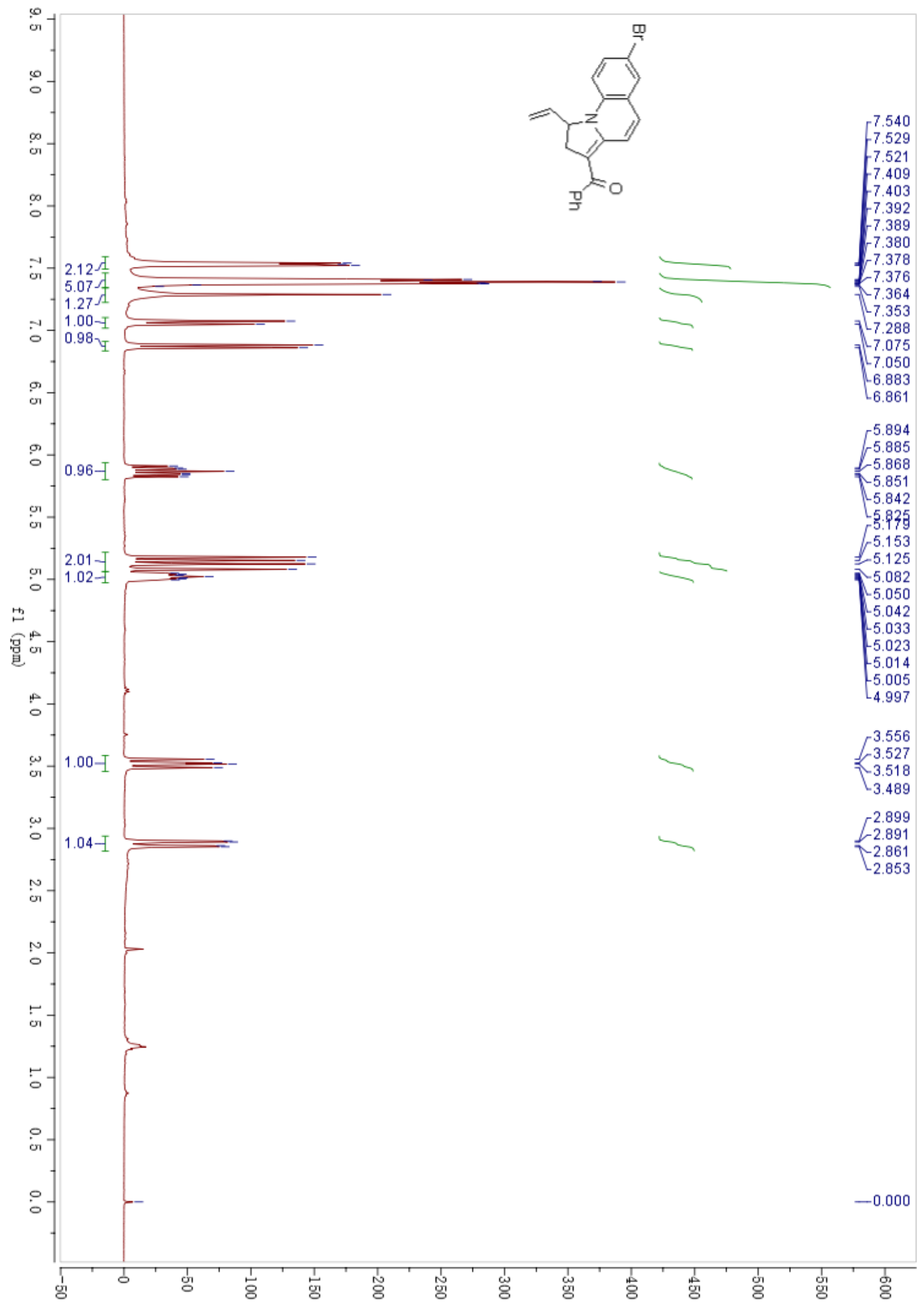


${ }^{13} \mathrm{C}$ NMR spectra of $\mathbf{3 o}$

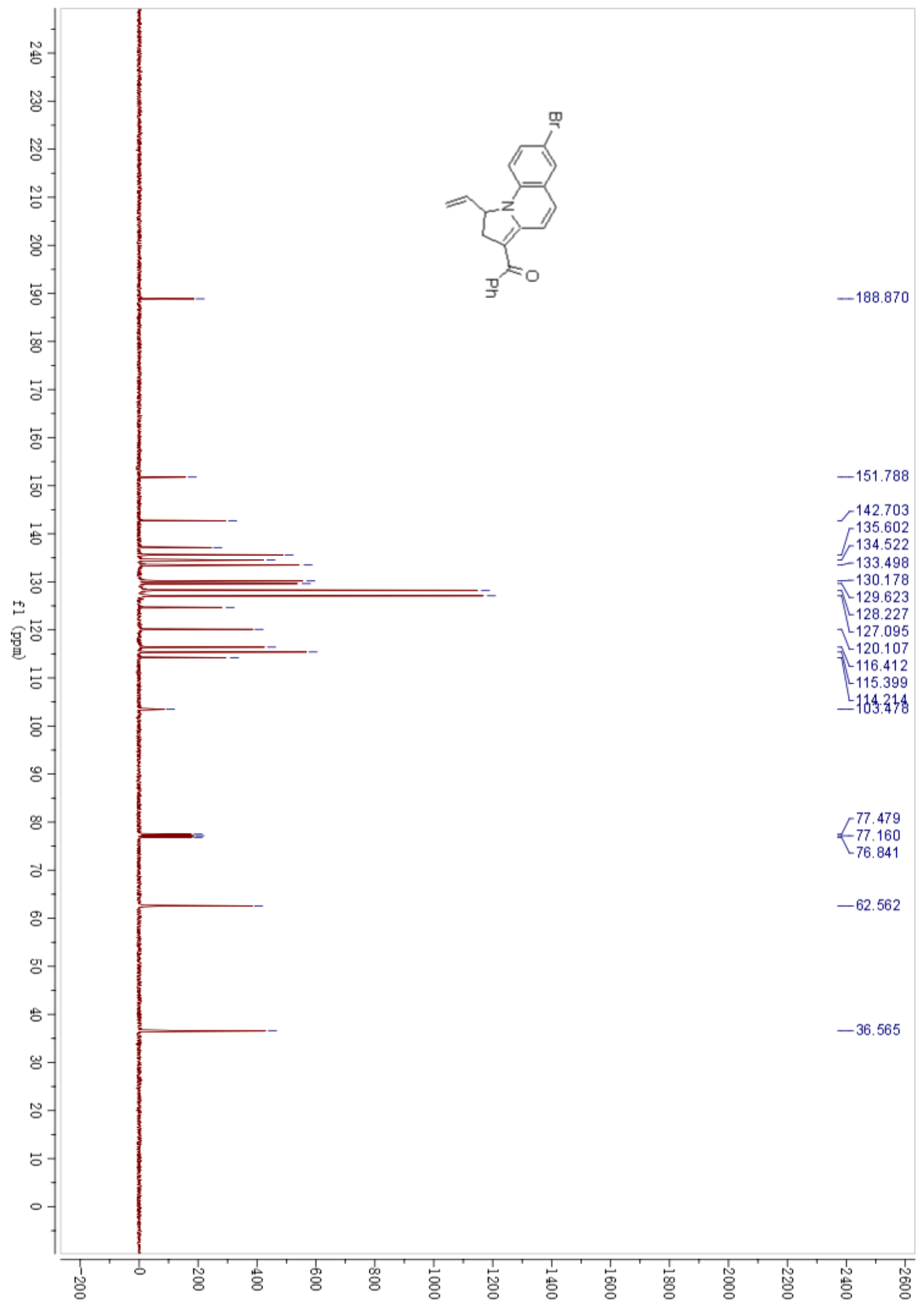


${ }^{1} \mathrm{H}$ NMR spectra of $\mathbf{3 p}$

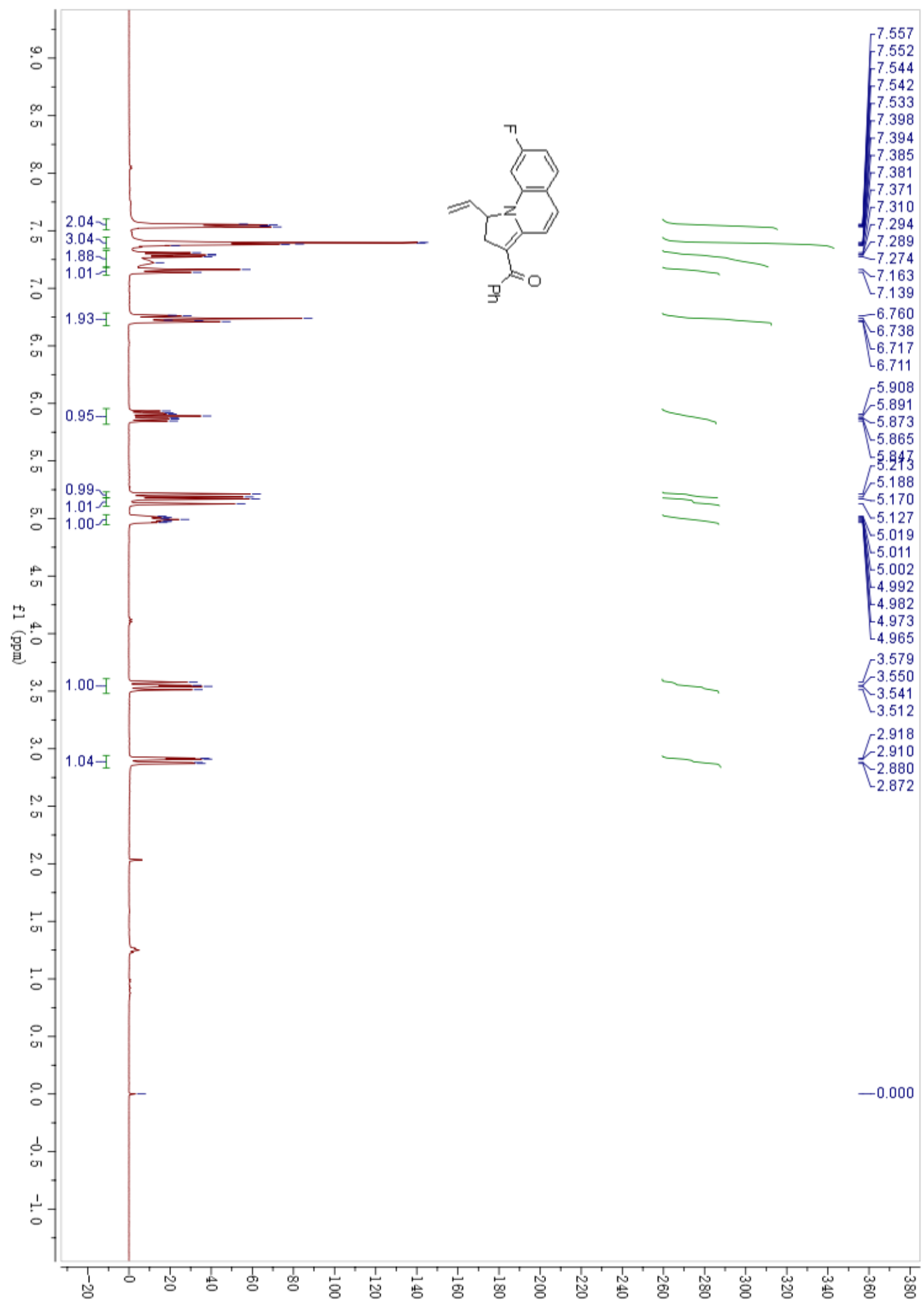


${ }^{13} \mathrm{C}$ NMR spectra of $\mathbf{3 p}$

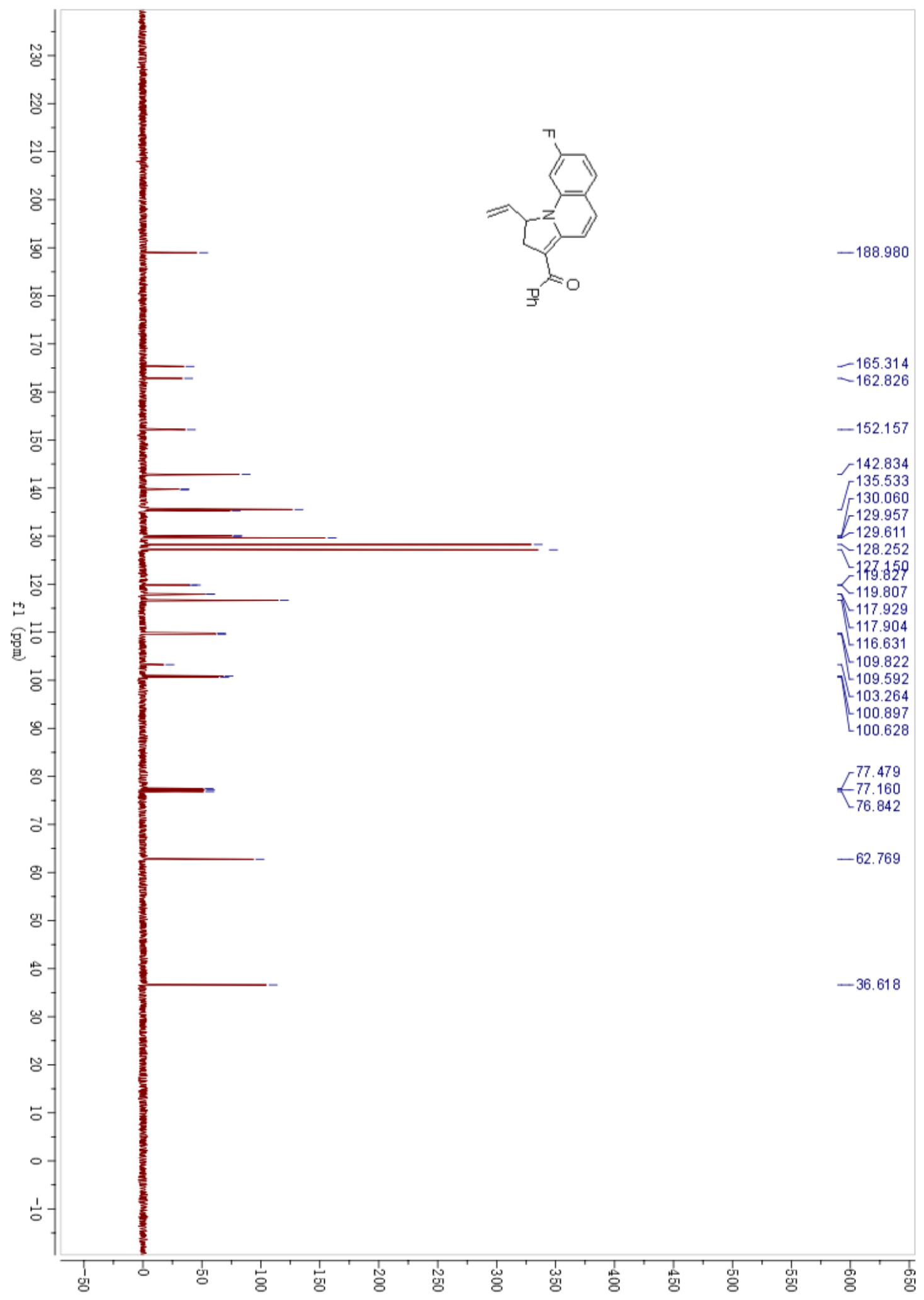


${ }^{19}$ F NMR spectra of $\mathbf{3 p}$

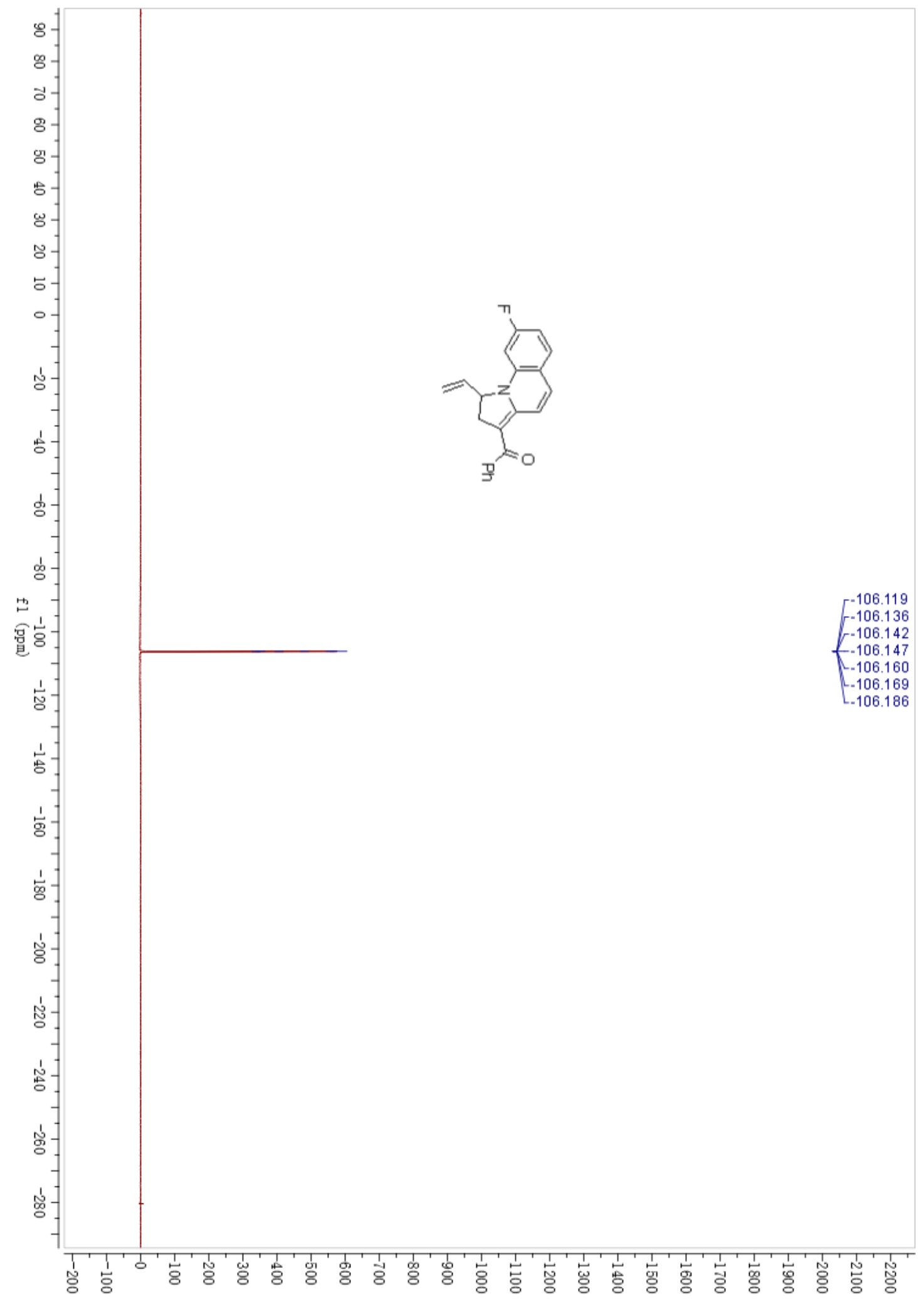


${ }^{1} \mathrm{H}$ NMR spectra of $\mathbf{3 q}$

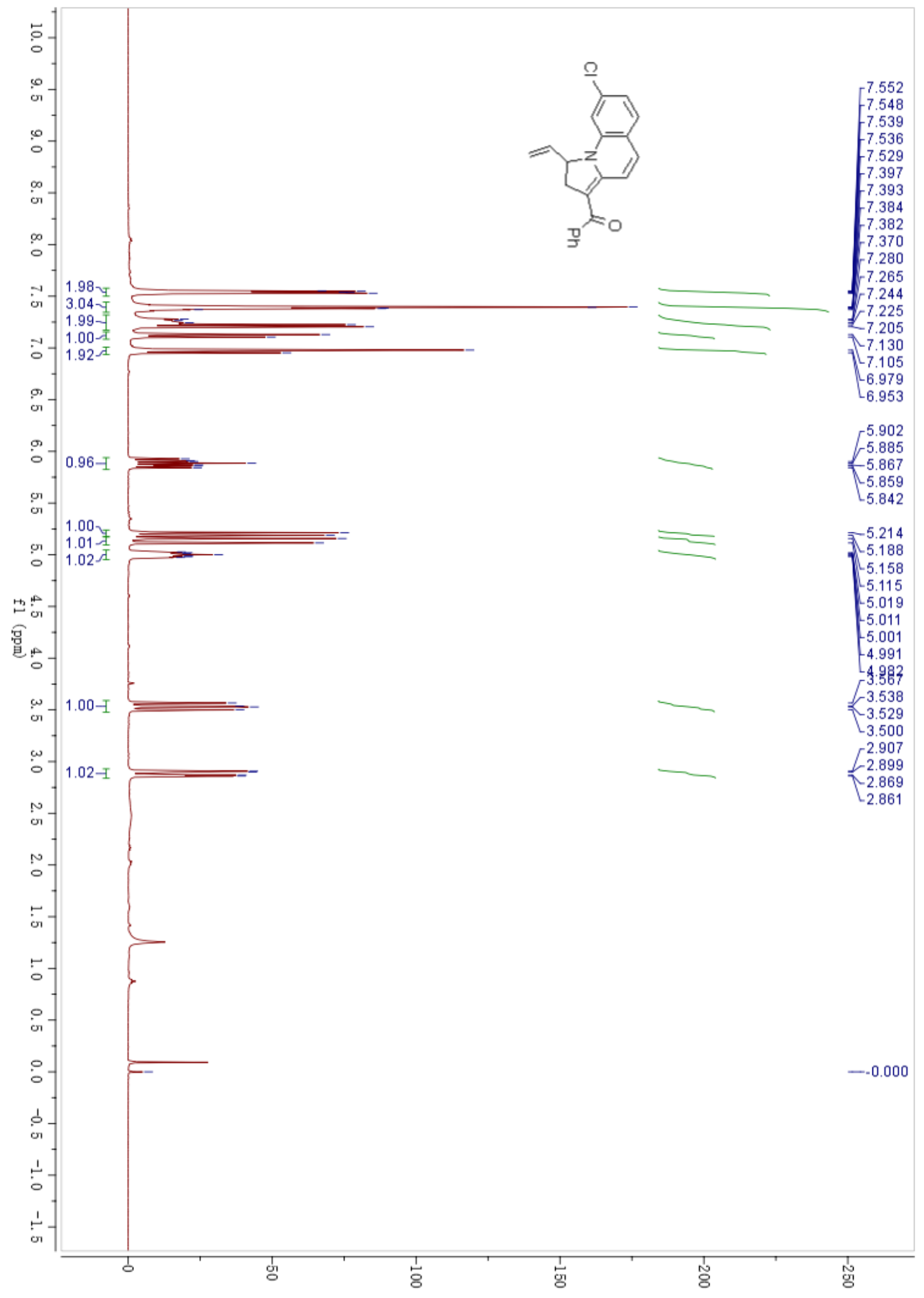


${ }^{13} \mathrm{C}$ NMR spectra of $\mathbf{3 q}$

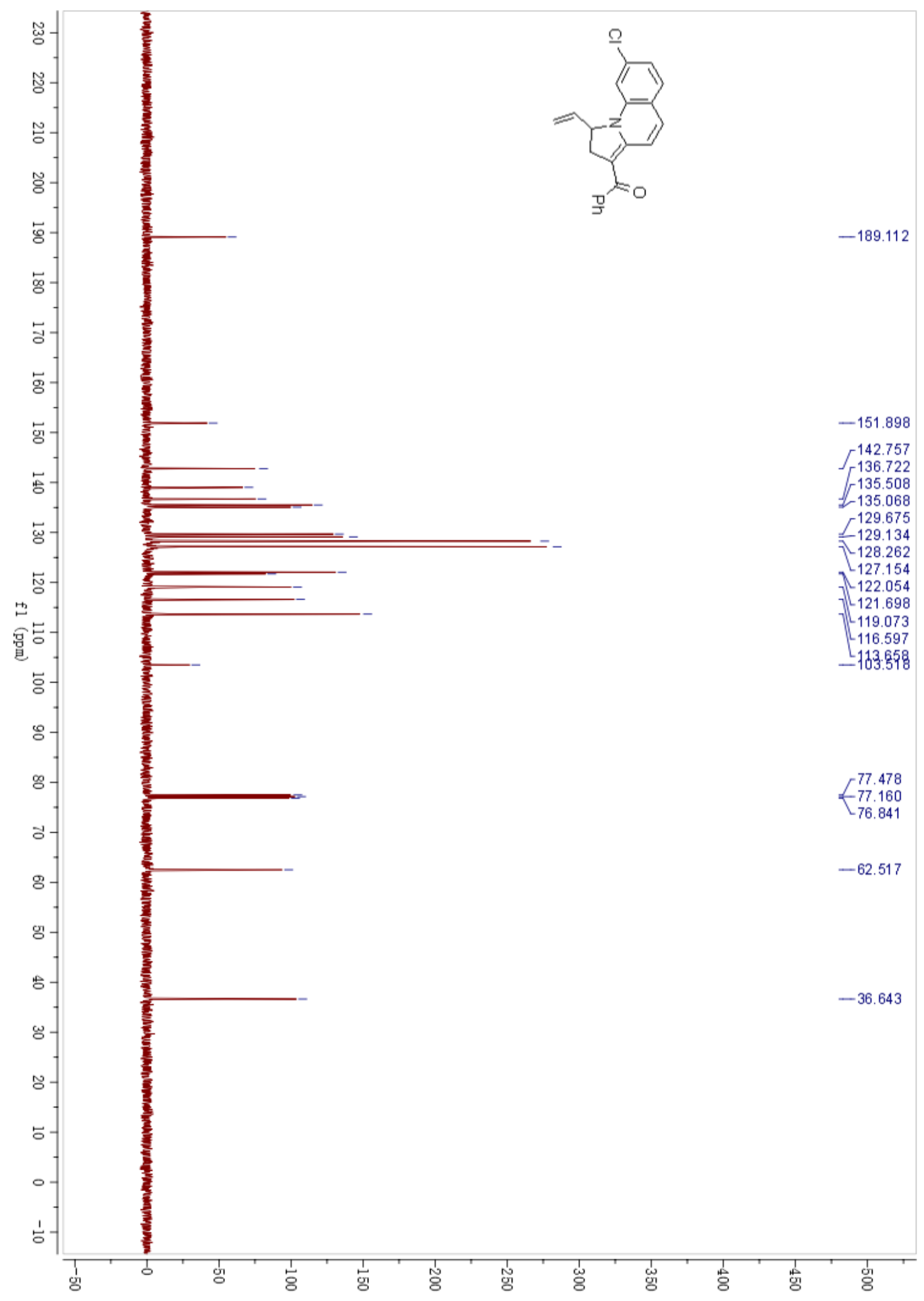


${ }^{1} \mathrm{H}$ NMR spectra of $\mathbf{3 r}$

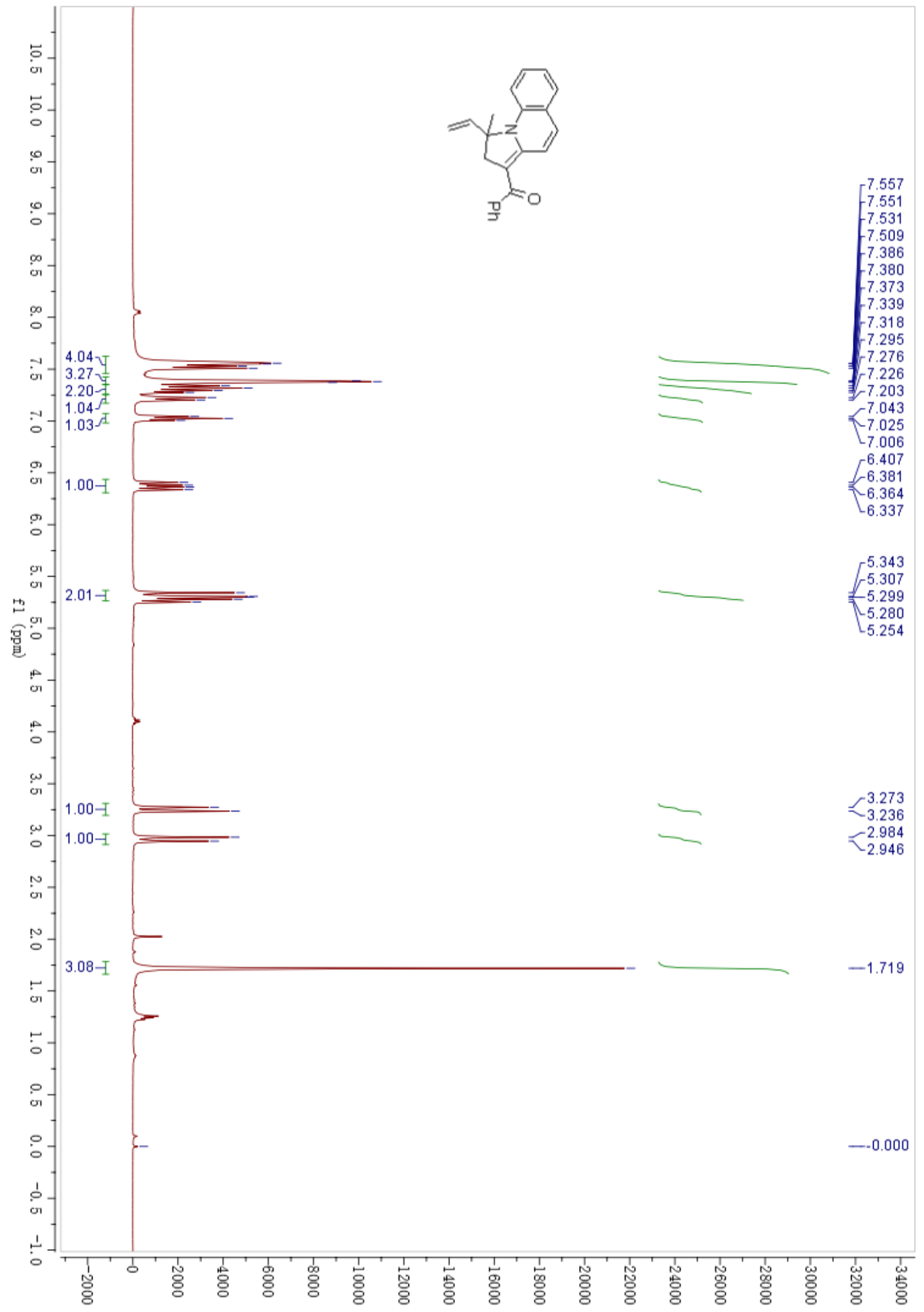


${ }^{13} \mathrm{C}$ NMR spectra of $\mathbf{3 r}$

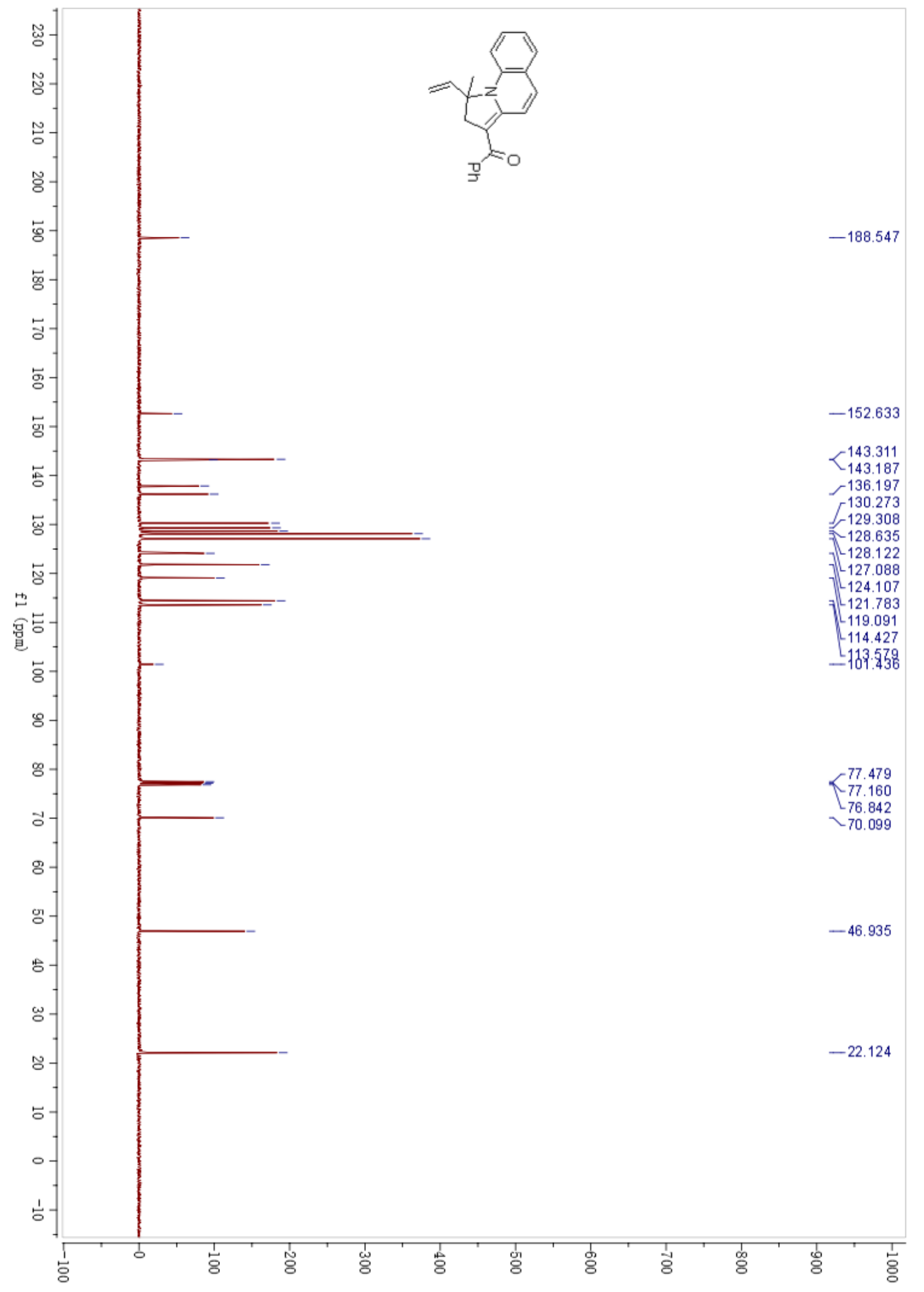


${ }^{1} \mathrm{H}$ NMR spectra of $\mathbf{3 s}$

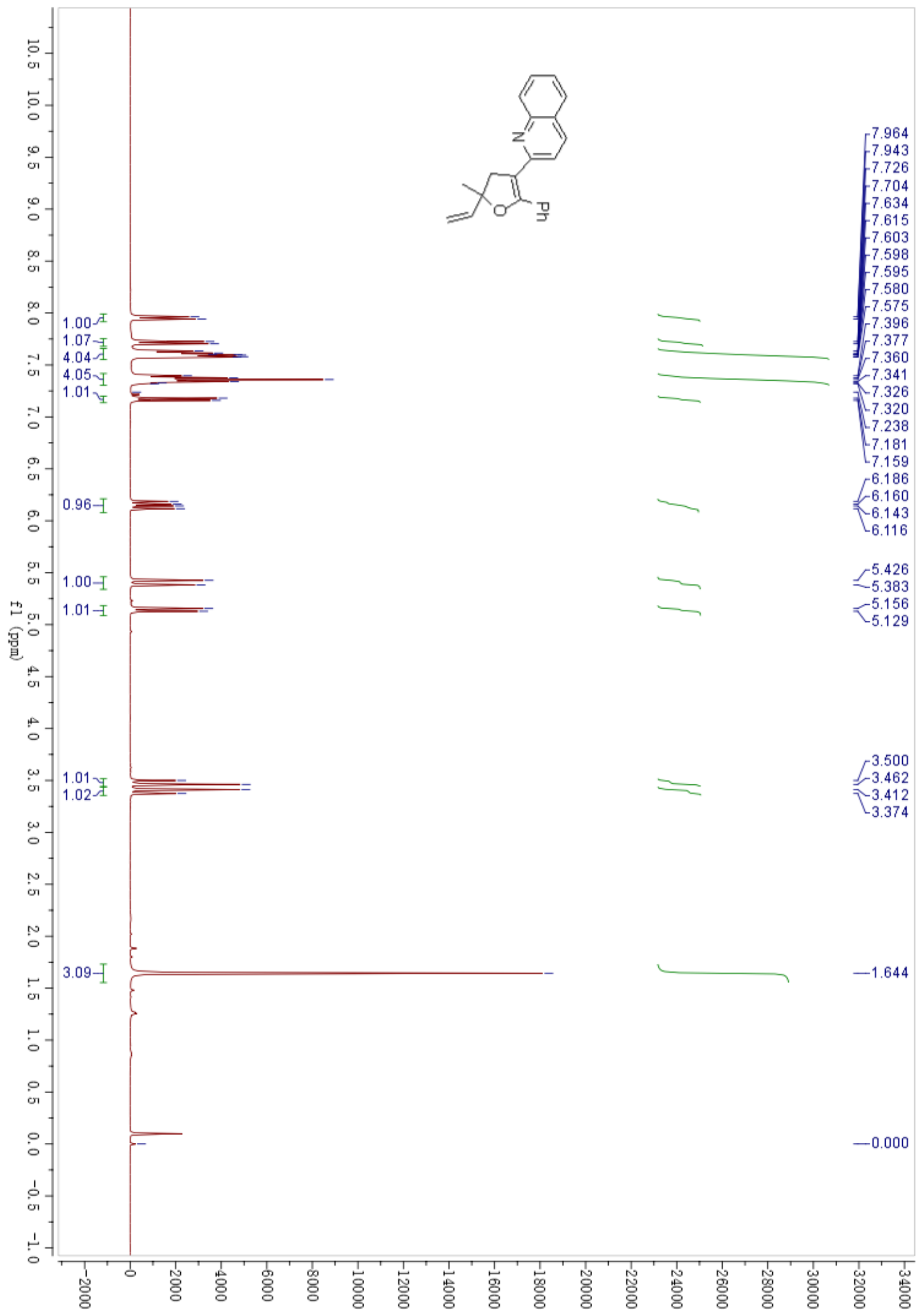


${ }^{13} \mathrm{C}$ NMR spectra of $\mathbf{3 s}$

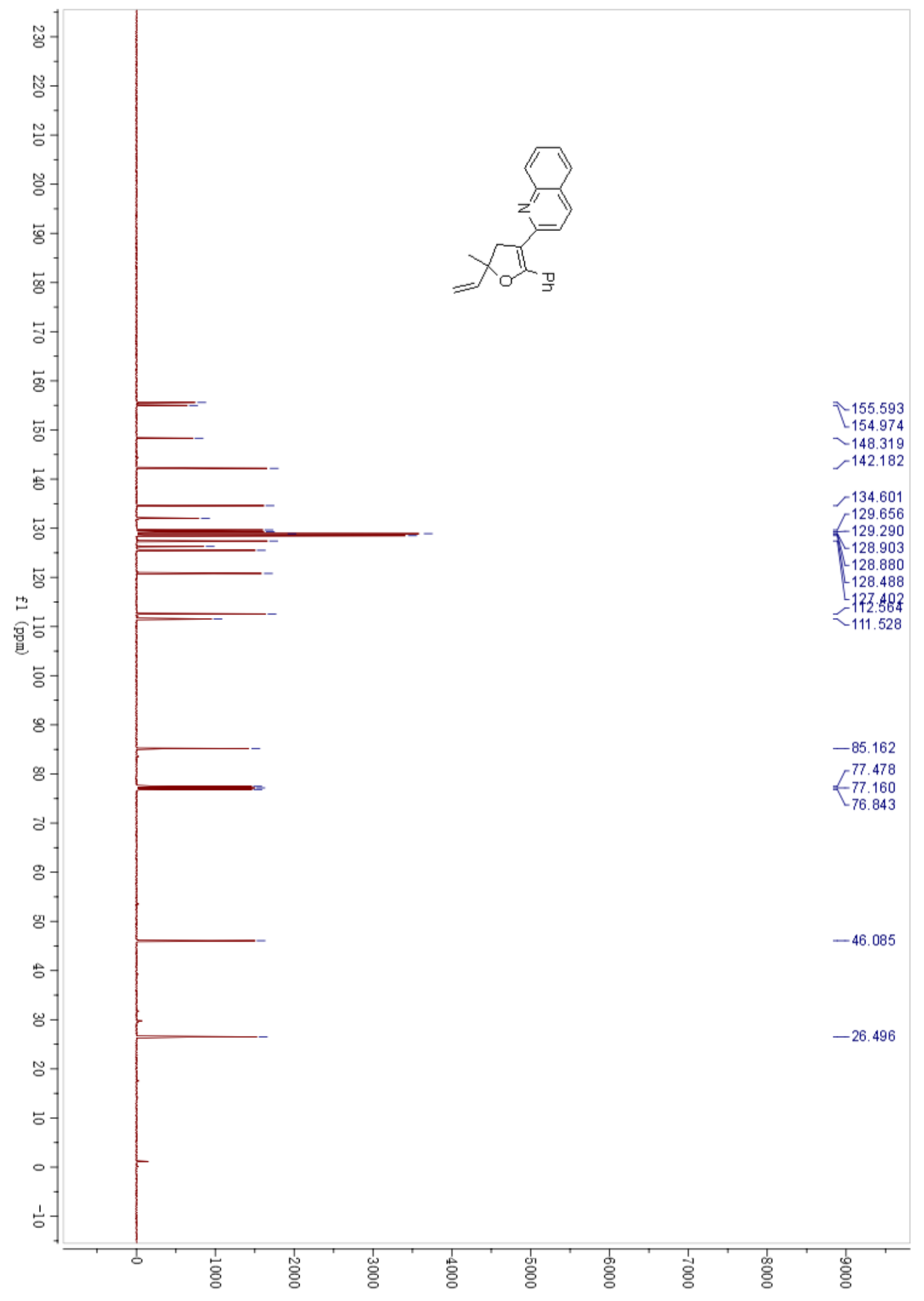


${ }^{1}$ H NMR spectra of $\mathbf{S 4}$

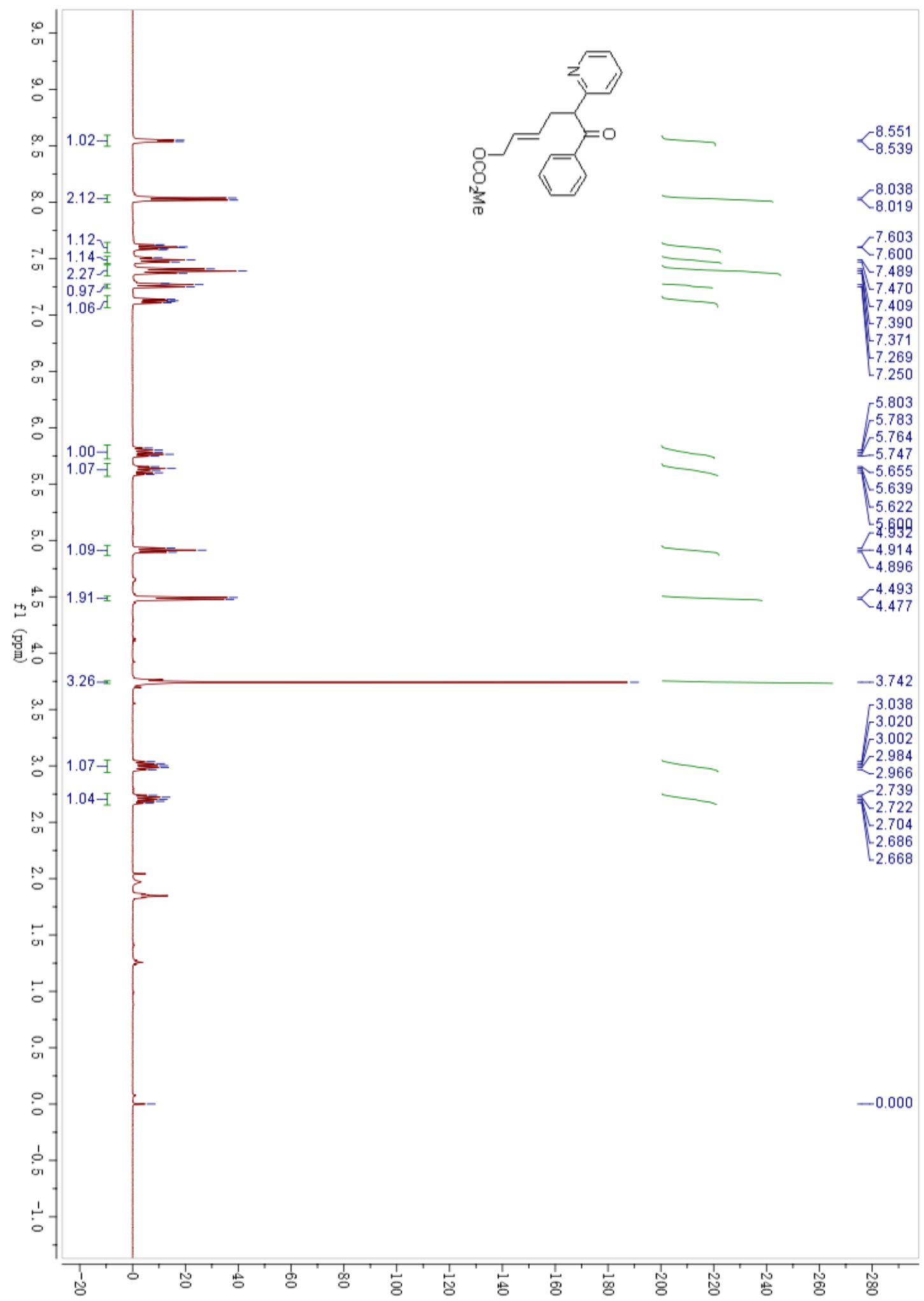




\section{References}

[1] Muir, C. W.; Kennedy, A. R.; Redmond, J. M.; Watson, A. J. B. Org. Biomol. Chem. 2013, 11, 3337.

[2] Wang, T.-L.; Ouyang, G.; He, Y.-M.; Fan, Q.-H. Synlett 2011, 7, 939.

[3] Wuts, P. G. M.; Ashford, S. W.; Anderson, A. M.; Atkins, J. R. Org. Lett. 2003, 5,1483 . 